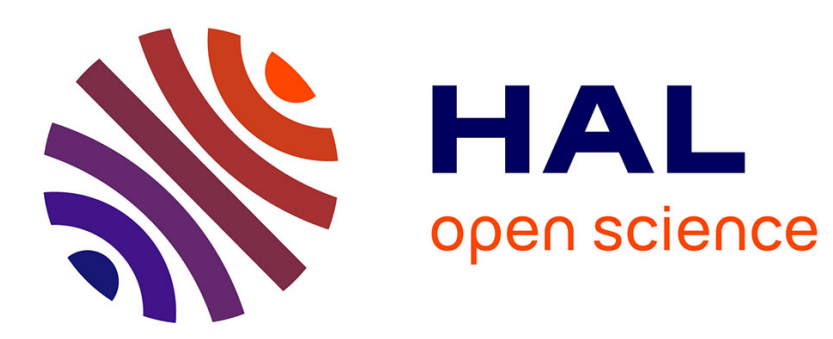

\title{
On the smoothness of the potential function in Riemannian optimal transport \\ Philippe Delanoë
}

\section{To cite this version:}

Philippe Delanoë. On the smoothness of the potential function in Riemannian optimal transport. Communications in Analysis and Geometry, 2015, 23 (1), pp.11-89. 10.4310/CAG.2015.v23.n1.a2 . hal-00957161v2

\section{HAL Id: hal-00957161 \\ https://hal.univ-cotedazur.fr/hal-00957161v2}

Submitted on 15 Mar 2014

HAL is a multi-disciplinary open access archive for the deposit and dissemination of scientific research documents, whether they are published or not. The documents may come from teaching and research institutions in France or abroad, or from public or private research centers.
L'archive ouverte pluridisciplinaire HAL, est destinée au dépôt et à la diffusion de documents scientifiques de niveau recherche, publiés ou non, émanant des établissements d'enseignement et de recherche français ou étrangers, des laboratoires publics ou privés. 


\title{
On the smoothness of the potential function in Riemannian optimal transport
}

\author{
to Neil Trudinger \\ Philippe Delanoë*
}

\begin{abstract}
On a closed Riemannian manifold, McCann proved the existence of a unique Borel map pushing a given smooth positive probability measure to another one while minimizing a related quadratic cost functional. The optimal map is obtained as the exponential of the gradient of a $c$-convex function $u$. The question of the smoothness of $u$ has been intensively investigated. We present a self-contained PDE approach to this problem. The smoothness question is reduced to a couple of $a$ priori estimates, namely: a positive lower bound on the Jacobian of the exponential map (meant at each fixed tangent space) restricted to the graph of grad $u$; and an upper bound on the $c$-Hessian of $u$. By the Ma-Trudinger-Wang device, the former estimate implies the latter on manifolds satisfying the so-called A3 condition. On such manifolds, it only remains to get the Jacobian lower bound. We get it on simply connected positively curved manifolds which are, either locally symmetric, or 2-dimensional with Gauss curvature $C^{2}$ close to 1.
\end{abstract}

2010 MSC: 35B65 (primary); 35B45, 35J96, 53C21 (secondary)

Keywords: Riemannian geometry, optimal transport, quadratic cost function, Monge-Ampère equation, classical solution, continuity method, a priori estimates, Jacobi fields

Author's address: Univ. Nice Sophia Antipolis, LJAD, UMR 7351, 06100 Nice, France, Author's E-mail: Philippe.Delanoe@unice.fr

\section{Contents}

1 Introduction $\quad 3$

1.1 The smoothness issue . . . . . . . . . . . . 3

1.2 Known results . . . . . . . . . . . . . . . 4

1.3 A stability conjecture . . . . . . . . . . . . . 6

1.4 Statement of results, outline of the paper $\ldots \ldots \ldots$

1.5 Acknowledgments . . . . . . . . . . . . . 9

${ }^{*}$ supported by the C.N.R.S. at UNS 
2 Preliminary $\quad 10$

2.1 Partial differentials . . . . . . . . . . . . . . . . 10

2.1.1 Real functions . . . . . . . . . . . . . . 10

2.1.2 Exponential map . . . . . . . . . . . . . . 13

2.1.3 Jacobi endomorphism . . . . . . . . . . . . 13

2.1.4 Linear connections on vector bundles . . . . . . . . . . 14

$2.2 C^{\ell}$ norms . . . . . . . . . . . . . . . . . 16

2.3 Jacobi fields and applications . . . . . . . . . . . . 16

2.3 .1 Jacobi equation . . . . . . . . . . . . . . 16

2.3.2 Applications . . . . . . . . . . . . . 17

2.4 Jacobians . . . . . . . . . . . . . . . . . 22

2.4.1 Calibrated determinants . . . . . . . . . . . 22

2.4.2 Exponential map at a point . . . . . . . . . . 23

2.4.3 Maps between Riemannian manifolds . . . . . . . . . . 24

2.4.4 Maps factorizing through nonconjugating vector fields 25

3 Foundations of the PDE approach 25

4 Continuity method $\quad 30$

4.1 Openess part of the method . . . . . . . . . . . 30

4.2 Closedness part of the method . . . . . . . . . . . . 32

4.3 Conclusion: proof of the general smoothness theorem . . . . 36

5 Continuity method applied to flat manifolds 38

6 Continuity method applied to A3 manifolds 38

6.1 Main statements . . . . . . . . . . . . . . . . . 38

6.2 Maximum Principle à la Ma-Trudinger-Wang . . . . . . . . . 40

7 Proofs completion: first order (stay-away) estimates 44

7.1 Positively curved Riemannian locally symmetric spaces . . . . 44

7.2 Nearly spherical surfaces . . . . . . . . . . . . . . . . . 47

7.2.1 Two-dimensional perturbation tools . . . . . . . 48

7.2.2 Analytic set up at the minimum point . . . . . . . . 53

7.2.3 Second order terms calculations . . . . . . . . . . 56

7.2.4 Third order terms calculations . . . . . . . . 57

7.2.5 Minimum condition: final discussion . . . . . . . . 61

A Assumptions comparison: curvature closeness versus metric closeness 


\section{Introduction}

Monge considered the problem of minimizing the total work necessary to push, in the Euclidean space, an assigned mass distribution to another one [Mon81]. Brenier revived the problem by replacing the total work by the total kinetic energy [Bre87, Bre91]. His theory was subsequently developed in the Riemannian setting by McCann [McC01] who obtained the following landmark result:

Theorem 1.1. Let $M$ be a closed Riemannian manifold and $\left(\mu_{0}, \mu_{1}\right), a$ couple of probability measures on $M$, both absolutely continuous with respect to the volume measure $d V$. There exists a unique minimizer of the total cost functional $\mathcal{C}(\phi)=\int_{M} c(m, \phi(m)) d \mu_{0}(m)$, among Borel maps $\phi: M \rightarrow M$ pushing $\mu_{0}$ to $\mu_{1}$. Moreover, if we compose that minimizer with the one obtained by switching $\mu_{0}$ and $\mu_{1}$, we get the identity at almost all points.

Here, we have called closed (for short) a manifold which is compact connected without boundary, and we have taken for cost function $c$ the twopoint function $\frac{1}{2} d^{2}$, where $d$ stands for the Riemannian distance function. The pushing condition means that $\mu_{1}(B)=\mu_{0}\left(\phi^{-1}(B)\right)$, for every Borel subset $B \subset M$, or else, that

$$
\int_{M} h d \mu_{1}=\int_{M}(h \circ \phi) d \mu_{0}
$$

for every $d V$ measurable function $h: M \rightarrow \mathbb{R}$. It is denoted by: $\phi_{\#} \mu_{0}=\mu_{1}$. In the sequel, it will be convenient to call abusively Monge's problem the minimization problem described in the above statement and to denote by $\operatorname{Mc}\left(\mu_{0}, \mu_{1}\right)$ its solution.

\subsection{The smoothness issue}

The solution map $\operatorname{Mc}\left(\mu_{0}, \mu_{1}\right)$ has a special form, obtained by composing the gradient field of a $c$-convex potential function $u$ with the exponential map of the manifold. To explain what $c$-convex means, one should first define the $c$-supremal convolution of a function $v: M \rightarrow \mathbb{R} \cup\{+\infty\}$ bounded at some point, as the function $v^{c}$ of the same kind, given by: $v^{c}(m)=$ $\sup _{p \in M}(-c(m, p)-v(p))$. We say that $u$ is $c$-convex if it is equal to $v^{c}$ for a $p \in M$

function $v$ of the preceding type. If so, the function $u$ satisfyies: $u=\left(u^{c}\right)^{c}$. In [McC01], McCann shows that $c$-convex functions must be Lipschitz, hence almost everywhere differentiable, and that their gradients are Borel maps. Furthermore, if the map $\operatorname{Mc}\left(\mu_{0}, \mu_{1}\right)$ is equal to $G_{u}:=\exp (\operatorname{grad} u)$ with $u$ $c$-convex, the inverse minimizer $\operatorname{Mc}\left(\mu_{1}, \mu_{0}\right)$ is given by $G_{u^{c}}$. The pair of solution potentials $\left(u, u^{c}\right)$ itself is constructed by Kantorovich duality (see $[\mathrm{McC} 01])$. 
The potential function $u=u\left(\mu_{0}, \mu_{1}\right)$ of the map $\operatorname{Mc}\left(\mu_{0}, \mu_{1}\right)=G_{u}$ is called a Brenier solution of the Monge-Ampère equation:

$$
\rho_{1}\left(G_{u}(m)\right) \operatorname{Jac}\left(G_{u}\right)(m)=\rho_{0}(m)
$$

where the $\rho_{i}$ 's stand for the Radon-Nikodym derivatives $\frac{d \mu_{i}}{d V}$, and Jac, formally for the Jacobian of the map $G_{u}: M \rightarrow M$ (see Section 2.4 .3 below). The very meaning of $\operatorname{Jac}\left(G_{u}\right)$ in the present case is specified in [CMS01]. This equation is nothing but the change of variables formula which one would expect from the pushing condition (1.1) for the invertible map $\phi=G_{u}$. If the given densities $\rho_{0}, \rho_{1}$ are $L^{\infty}$ and positively pinched, possibly endowed with some degree of smoothness, can we infer that the Brenier solution $u\left(\mu_{0}, \mu_{1}\right)$ is correspondingly smooth ? Looking for sharp conditions on the data $M, \mu_{0}, \mu_{1}$ under which the potential function $u\left(\mu_{0}, \mu_{1}\right)$ must be $C^{1}$ has been a topic of intense research in the recent years [FR09, KM12, FRV11, FKM13b, FKM13a, Vét11]. Higher order smoothness of $u\left(\mu_{0}, \mu_{1}\right)$ was derived in the cases listed below, relying on the Ma-Trudinger-Wang estimate [MTW05] or its subsequent improvements [TW09, LTW10], combined with a so-called stay-away estimate.

Henceforth, we always work on a closed Riemannian manifold $M$, we fix a couple $(k, \alpha) \in \mathbb{N} \times(0,1)$, with $k \geqslant 2$, and we assume that the given probability densities $\rho_{0}$ and $\rho_{1}$ are $C^{k, \alpha}$ nonvanishing on $M$. For short, we say that the given measures $\mu_{0}, \mu_{1}$ are $C^{k, \alpha}$ positive. We want to prove that the potential function $u\left(\mu_{0}, \mu_{1}\right)$ is $C^{k+2, \alpha}$. This is the question which motivates the present paper.

\subsection{Known results}

If the manifold $M$ is flat, the potential function $u\left(\mu_{0}, \mu_{1}\right)$ must be $C^{k+2, \alpha}$. Using the Bieberbach theorem (see e.g. [KN96a, section V.4]) combined with the covering space result of [DG10, Appendix C], this result is readily seen to follow from Cordero-Erausquin's regularity theorem [Cor99] and further elliptic regularity. The flat case lies on a borderline, though. Indeed, as soon as the sectional curvature function $K$ is allowed to take a negative value at some tangent 2-plane, one can select smooth probability densities $\rho_{0}, \rho_{1}$ such that the map $\operatorname{Mc}\left(\mu_{0}, \mu_{1}\right)$ is not continuous [Loe09]. If $M$ has constant positive curvature, the potential function $u\left(\mu_{0}, \mu_{1}\right)$ must be smooth [Loe11, DG10]. But the assumption $K>0$ by itself does not imply the same result; counterexamples can be constructed in any dimension, again with $\operatorname{Mc}\left(\mu_{0}, \mu_{1}\right)$ not continuous [Kim08] (see also [LV10] in the light of [FRV11]). Trudinger conjectured that the smoothness issue should be tractable anytime the curvature of $M$ is positive and slowly varying [Tru06] (see also [DG10, p.67]). We thus expect that the class of positively curved 
Riemannian locally symmetric spaces be a privileged one. If $M$ (or its universal cover) is a complex or quaternionic projective space, the potential function $u\left(\mu_{0}, \mu_{1}\right)$ must be $C^{k+2, \alpha}$ [KM12, FKM13a, LTW10]. Sticking to Riemannian local symmetry, Figalli, Kim and McCann further took for $M$ the Riemannian product of constant curvature spheres of various dimensions. This case is, again, on the borderline for optimal transport smoothness, since the sectional curvature of $M$ is now just nonnegative. Their results in that case [FKM13b, FKM13a], combined with an extension (yet unpublished) of the interior regularity theorem of [LTW10], imply that the potential function $u\left(\mu_{0}, \mu_{1}\right)$ is $C^{k+2, \alpha}$. Finally, in Corollary 2 of [DG10], dropping local symmetry, we treated the case of nearly spherical manifolds with nontrivial topology. Recalling that $K: \operatorname{Gr}_{2}(M) \rightarrow \mathbb{R}$ stands for the sectional curvature function defined on the Grassmann bundle of tangent 2-planes, and denoting by $R$ the Riemann tensor of $M$, by $\nabla$, the Levi-Civita connection and by $\left|\nabla^{k} R\right|$, the Riemannian norm of the tensor $\nabla^{k} R$, we can state the main result of [DG10] as follows:

Theorem 1.2. Let $M$ be a closed positively curved Riemannian manifold with curvature normalized by $\min _{\mathrm{Gr}_{2}(M)} K=1$. Assume that $M$ has a nontrivial first fundamental group. We can find a universal constant $\epsilon>0$ such that, if the functions $(K-1),|\nabla R|$ and $\left|\nabla^{2} R\right|$ are everywhere smaller than $\epsilon$, given any couple $(k, \alpha) \in \mathbb{N} \times(0,1)$, with $k \geqslant 2$, the potential function of the optimal map $\mathrm{Mc}\left(\mu_{0}, \mu_{1}\right)$ must be $C^{k+2, \alpha}$ for every couple $\left(\mu_{0}, \mu_{1}\right)$ of $C^{k, \alpha}$ positive probability measures.

In the simply connected case, that is, with $M$ diffeomorphic to $\mathbb{S}^{n}$, under the stronger ${ }^{1}$ condition that the metric of $M$ be $C^{4}$ close to a constant curvature metric, the continuity of $\operatorname{Mc}\left(\mu_{0}, \mu_{1}\right)$ was established in [FR09, FRV10, FRV12, FRV11]. The higher order smoothness of the potential function $u\left(\mu_{0}, \mu_{1}\right)$ remains to be proven in that case, due to an incomplete stay-away estimate. Specifically, Villani and his coworkers miss to control how far the image point $\operatorname{Mc}\left(\mu_{0}, \mu_{1}\right)(m)$ stays away from the first conjugate point $p$ on the geodesic ray $t \in[0,+\infty) \rightarrow \exp _{m}\left(t \operatorname{grad} u\left(\mu_{0}, \mu_{1}\right)(m)\right) \in M$, in case there is no other minimizing geodesic from $m$ to $p$. The condition $\delta(M)>0$ of [LV10, Theorem 7.1] was designed to exclude that case. It is verified on constant curvature spheres but unrealistic when the curvature is perturbed. The stay-away property, in the form just stated, is essential for the smoothness issue (see the first requirement of Theorem 1.5). The nondegeneracy of the Monge-Ampère equation (1.2), as an elliptic equation, is at stake. This can already be seen from the symbol of the first order differential operator obtained by linearizing formally the map $u \mapsto G_{u}=$ $\exp (\operatorname{grad} u)$. This symbol, calculated at a point $m \in M$, is equal to the linear map $\xi \in T_{m}^{*} M \rightarrow d\left(\exp _{m}\right)(\operatorname{grad} u(m))\left(\xi^{\sharp}\right) \in T_{G_{u}(m)} M$, where $\xi \in$

\footnotetext{
${ }^{1}$ see the appendix of the paper
} 
$T_{m}^{*} M \rightarrow \xi^{\sharp} \in T_{m} M$ stands for the Riesz isomorphism associated to the Euclidean scalar product on $T_{m} M$. The symbol is an isomorphism if and only if the image point $G_{u}(m)$ does not reach the first conjugate point on the ray $t \in[0,+\infty) \rightarrow \exp _{m}(t \operatorname{grad} u(m)) \in M$.

\subsection{A stability conjecture}

In the spirit of Trudinger's conjecture, one should be able to improve Theorem 1.2 by allowing the triviality of $\pi_{1}(M)$ and by dropping the $\epsilon$ curvature pinching condition, that is, to treat the nearly Riemannian locally symmetric case. Besides, the smoothness result of [FKM13b] indicates that one should even be able to let the curvature $K$ be merely nonnegative. In other words, we are led to state the following:

Conjecture 1. Let $M$ be a closed nonnegatively curved Riemannian manifold. If the $C^{1}$ norm of the tensor $\nabla R$ is sufficiently small, given any couple $(k, \alpha) \in \mathbb{N} \times(0,1)$, with $k \geqslant 2$, the potential function of the optimal map $\operatorname{Mc}\left(\mu_{0}, \mu_{1}\right)$ must be $C^{k+2, \alpha}$ for every couple $\left(\mu_{0}, \mu_{1}\right)$ of $C^{k, \alpha}$ positive probability measures.

In the present paper, we will provide a method (see Theorem 1.5 below) for attacking this program and we will test it successfully in two cases, namely: the locally symmetric case (Theorem 1.3), which is the starting point of the conjecture (letting $\epsilon=0$ ), and the 2-sphere case (Theorem 1.4). With this method at hand, much work still lies ahead to prove the full conjecture (we hope to return to part of it soon).

\subsection{Statement of results, outline of the paper}

We are going to prove the following results:

Theorem 1.3. If the manifold $M$ is positively curved Riemannian locally symmetric, given any couple $(k, \alpha) \in \mathbb{N} \times(0,1)$, with $k \geqslant 2$, the potential function of the optimal map $\operatorname{Mc}\left(\mu_{0}, \mu_{1}\right)$ must be $C^{k+2, \alpha}$ for every couple $\left(\mu_{0}, \mu_{1}\right)$ of $C^{k, \alpha}$ positive probability measures.

Theorem 1.4. We can find a universal constant $\epsilon>0$ such that, on the manifold $M=\mathbb{S}^{2}$ endowed with a Riemannian metric of Gauss curvature $K$ normalized by $\min _{M} K=1$ and satisfying the bound $|K-1|_{C^{2}(M)} \leqslant \epsilon$, given any couple $(k, \alpha) \in \mathbb{N} \times(0,1)$, with $k \geqslant 2$, the potential function of the optimal map $\operatorname{Mc}\left(\mu_{0}, \mu_{1}\right)$ must be $C^{k+2, \alpha}$ for every couple $\left(\mu_{0}, \mu_{1}\right)$ of $C^{k, \alpha}$ positive probability measures.

As mentioned above, Theorem 1.3 was known except for the case of the Cayley plane. Here, we will give a unified proof of it. Theorem 1.4 is new. In connection with the comment made after Theorem 1.2 , let us mention 
that, for every Riemannian metric on the 2-sphere, the cut locus of each point must contain a conjugate point [Mye35, Wei68]. The proofs of these two theorems share a common part (sections 3,4 and 6) valid for every A3 manifold as stated in Theorem 6.1 below. They depart only by their final step (section 7) consisting of a suitable stay-away estimate (all these notions will be specified below).

We use a PDE method which makes no use of McCann's potential function $u\left(\mu_{0}, \mu_{1}\right)$, except for its final identification with a smooth potential function $u$ obtained as a classical solution of the Monge-Ampère equation (1.2). This method was partly sketched, with some gaps, in [Del04] and used in [Loe11, DG10]. It is presented here for the first time in a complete self-contained form. It involves only techniques of differential geometry combined with an intrinsic local analysis of critical and extremum differential conditions, in contrast with smoothness proofs given on closed manifolds, as yet, by other authors. It yields a smoothness scheme for the solution of (1.2) valid on every closed Riemannian manifold as stated in Theorem 1.5 below. The strategy of the proof may be described by the following steps.

Section 3. The classical solvability of the Monge-Ampère equation (1.2) completely encodes the smoothness issue for Monge's problem: specifically, we show that the existence of a $C^{2}$ solution of (1.2) is equivalent to the optimal potential $u\left(\mu_{0}, \mu_{1}\right)$ being a $C^{2}$ function. Moreover, at such a $C^{2}$ solution, the Monge-Ampère equation must be non-degenerate and elliptic.

Section 4. We solve uniquely (1.2) by the continuity method, replacing the target probability density $\rho_{1}$ by the deformed one $\rho_{t}=t \rho_{1}+(1-t) \rho_{0}$ and arguing by connectedness on the subset $\mathcal{T}$ of all values of $t \in[0,1]$ for which the resulting analogue of (1.2), namely the continuity equation (4.1), admits a classical solution $u_{t} \in C^{k+2, \alpha}(M, \mathbb{R})$. To ensure uniqueness, the solutions $u_{t}$ are normalized by $\int_{M} u_{t} d V=0$ (see Proposition 4.2). Showing that $\mathcal{T}$ coincides with $[0,1]$ boils down to proving that the supremum over $\mathcal{T}$ of the largest eigenvalue on the manifold $M$ of the Hessian endomorphism field $m \mapsto \nabla \operatorname{grad} u_{t}(m)$ is finite. This finiteness requirement turns out equivalent to requirements 1 and 2 of the general smoothness result to which the continuity method leads, namely:

Theorem 1.5 (general smoothness result). On a closed Riemannian manifold $M$, given $(k, \alpha) \in \mathbb{N} \times(0,1)$, with $k \geqslant 2$, the potential function $u\left(\mu_{0}, \mu_{1}\right)$ is $C^{k+2, \alpha}$ for every couple of $C^{k, \alpha}$ positive probability measures $\left(\mu_{0}, \mu_{1}\right)$, if, for each such couple, the following two requirements are fulfilled:

1) The infimum of $\min _{m \in M} \operatorname{Jac}\left(\exp _{m}\right)\left(\operatorname{grad} u_{t}(m)\right)$, over the solution counterset $\mathcal{T}$ just defined, is positive;

2) The supremum, over $\mathcal{T}$, of the largest eigenvalue on the manifold $M$ of the symmetric endomorphism field $m \mapsto \mathcal{J}\left(m, \operatorname{grad} u_{t}(m)\right)+\nabla \operatorname{grad} u_{t}(m)$, is finite ( $\mathcal{J}$ standing for the Jacobi endomorphism field, see section 2.1.3).

If, for a couple of measures $\left(\mu_{0}, \mu_{1}\right)$ of the above type, either requirements 
fails, there exists a real $\tau \in[0,1]$ such that, setting $\mu_{\tau}$ for the measure, still of the same type, equal to $\tau \mu_{1}+(1-\tau) \mu_{0}$, the potential function $u\left(\mu_{0}, \mu_{\tau}\right)$ is not $C^{2}$.

This statement is new. It will be established in the concluding part of section 4 . The proofs of Theorems 1.3 and 1.4 are now reduced to checking, on the corresponding manifolds, the two requirements stated in Theorem 1.5. For this purpose, we will have to derive from the Monge-Ampère equation satisfied by $u_{t}$, for $t \in \mathcal{T}$, a suitable sequence of uniform a priori estimates.

Remark 1.1 (specifications for the a priori estimates). The a priori estimates on $\left|\nabla^{\ell} u_{t}\right|(m)$ or on related quantities of order $\ell$ with respect to $u_{t}$, for $\ell=0,1,2$, will be understood to be independent of $(t, m) \in \mathcal{T} \times M$ and controlled, unless otherwise specified, solely by appropriate geometric invariants of the Riemannian manifold $M$ and by the largest $C^{\ell}$ norm of $\log \rho_{t}$ as $t \in[0,1]$. Any such estimate will be said under control. Here, we should warn the reader that these estimates, derived by maximum principle techniques, will not be as sharp as the ones designed for nonsmooth densities. This was already clear in the two contrasted parts of [DL06]. It is the price to pay for the self-consistency of a PDE method ignoring McCann's solution.

Checking the requirements 1 and 2 of Theorem 1.5 on each particular manifold will be achieved by carrying out the following two estimates:

Estimate 1 (first order). There exists a real $\delta>0$ under control such that:

$$
\operatorname{Jac}\left(\exp _{m}\right)\left(\operatorname{grad} u_{t}(m)\right) \geqslant \delta,
$$

for every point $(t, m) \in \mathcal{T} \times M$.

Estimate 2 (second order). There exists a real $\Lambda>0$ under control, possibly depending on the preceding constant $\delta$, such that:

$$
\mathcal{J}\left(m, \operatorname{grad} u_{t}(m)\right)+\nabla \operatorname{grad} u_{t}(m) \leqslant \Lambda \operatorname{Id}_{m},
$$

for every point $(t, m) \in \mathcal{T} \times M$ (where $\operatorname{Id}_{m}$ denotes the identity of $\left.T_{m} M\right)$.

The controls actually obtained on $\delta$ and $\Lambda$ will be further specified for each estimate (see Lemma 6.1 for $\Lambda$, and sections 7.1 and 7.2 for $\delta$ ).

Section 6. Estimate 1 implies Estimate 2 anytime the manifold $M$ fulfills the emblematic condition A3 discovered in [MTW05], as do the manifolds of Theorems 1.3 and 1.4 [DR13, DG11]. Indeed, this is the celebrated MaTrudinger-Wang estimate [MTW05, Section 4]. For completeness, the latter is recast below in intrinsic form, as a maximum principle for the trace of the tensor appearing in Estimate 2 (an alternative adaptation was proposed in [DG10, Appendix B]). So much for the common part of the proofs. 
Section 7. We complete the proofs by checking Estimate 1 separately for each theorem. For Theorem 1.3, we do it by proving a maximum principle for $\left|\operatorname{grad} u_{t}\right|^{2}$ which extends the one proved for constant curvature spheres in [DL06, section 3]. For Theorem 1.4, we must prove a genuine minimum principle for the full test-function $m \mapsto \operatorname{Jac}\left(\exp _{m}\right)\left(\operatorname{grad} u_{t}(m)\right)$ instead, getting for it a positive lower bound under control. This estimate is the newest result of the paper.

Finally, let us say a few words about the sections which we did not mention yet. In Section 5, we revisit the known case of flat manifolds ( $c f$. supra) since in that case, Estimate 1 trivially holds. Flat manifolds do not fulfill condition A3, nevertheless Estimate 2 holds as a particular case of an a priori estimate proven by the author [Del82, pp.428-429].

Last, but not least, Section 2 contains an appropriate introduction to the differential geometric tools which make natural the PDE approach. Admittedly, it is quite a long preliminary, but we could not find the right introduction in the literature. The heavy use of differential geometry for proving the smoothness of optimal potential functions can be conceived by noting that the operator $u \mapsto \operatorname{Jac}\left(G_{u}\right)$ factors through the exponential map. The background of Section 2 will serve to prove, for instance, the non-degeneracy and ellipticity of that operator, when restricted to the $C^{2}$ functions at which it does not vanish (see Proposition 3.1). Our efforts to construct a $C^{2}$ solution of the Monge-Ampère equation (1.2) will mostly bear on getting a control on that non-degeneracy, by Estimate 1, and that ellipticity via Estimate 2. These a priori estimates will require deeper tools from geometry again, like Proposition 7.1 for tackling Estimate 1, or like the verification of condition A3 [DR13, DG11] on which Estimate 2 relies via the Ma-Trudinger-Wang device. From the inescapable role played by differential geometry for solving (1.2) in the classical sense, we can realize that much work lies ahead in the research program mentioned above.

\subsection{Acknowledgments}

In October 2010 at the Fields Institute, I got convinced to write a detailed account on the PDE approach in Riemannian optimal transport by talking with Robert McCann. I wish to thank him for his keen interest. I reported on a preliminary draft of the present paper in the meeting on "Geometric and Nonlinear Partial Differential Equations" held in June 2012 in Xi'an (China), in honor of Neil Trudinger's 70th birthday. I discussed there with Alessio Figalli, reminding him of a pending question of mine, namely: can a smooth real function $u$ on a compact Riemannian manifold be such that the gradient-mapping $G_{u}$ pushes a smooth positive measure to another such one, without $u$ being $c$-convex ? From section 3 , we know that the answer is no whenever the function is $C^{2}$ and the measures, continuous. I am grateful to Figalli for communicating to me the first lemma of that section [Fig12]. 


\section{Preliminary}

Let $(M, g)$ be a closed unoriented $n$-dimensional Riemannian manifold. In the first part of this preliminary, we define partial differentials of functions or maps with source space the tangent bundle $\varpi_{M}: T M \rightarrow M$. It yields an intrinsic way of calculating the differential of a function on $M$, when given as the pullback by a vector field of a function defined on $T M$. Similar calculations are made for the exponential map and for the Jacobi endomorphism field. The second part of the preliminary bears on solutions of initial value problems for the Jacobi equation, focussing on their representation in terms of the partial differentials of the exponential map just developed. Applications are drawn, including a derivation of the main properties of the Jacobi endomorphism field. In the last part, we define Jacobian determinants for various types of maps arising in the smoothness problem under study.

\subsection{Partial differentials}

\subsubsection{Real functions}

Throughout the paper, we set $(m, V)$ for the generic point of $T M$, with the usual meaning that $m \in M$ is the base point and $V$, a tangent vector at $m$. Consistently with that notation, we would welcome a way of viewing uniquely every vector $W$, tangent to $T M$ at a point $(m, V)$, as a couple $W \simeq(\xi, \nu)$ of vectors in $T_{m} M \times T_{m} M$. Accordingly, we seek a global fashion of splitting the differential of a function $\psi: T M \rightarrow \mathbb{R}$ into the sum of two partial differentials, something like:

$$
d \psi(m, V)(W)=\frac{\partial \psi}{\partial m}(m, V)(\xi)+\frac{\partial \psi}{\partial V}(m, V)(\nu) .
$$

Let us explain how it can be done with some care ${ }^{2}$, using the Levi-Civita connection $^{3}$ of $M$. Once settled the case of real functions, we will be able to treat that of more general objects defined on $T M$. The present account is intended for non-experts. Other readers may skip it and just record the final equations (2.3) and (2.5).

In every natural chart $x=x(m), v=d x(m)(V)$ of $T M$, the differential of the function $\psi$ is given by

$$
d \psi(m, V)=\frac{\partial \psi}{\partial x^{i}}(x, v) d x^{i}+\frac{\partial \psi}{\partial v^{i}}(x, v) d v^{i},
$$

with Einstein's convention. In this local expression, only half of the terms are intrinsic. Indeed, the differential of the base point projection $\varpi_{M}$ :

\footnotetext{
${ }^{2}$ compare the preceding formula with the displayed one that precedes (2.3) below

${ }^{3}$ here, one could deal similarly with any vector bundle of finite rank over $M$, using a linear connection
} 
$T M \rightarrow M$ reads $d \varpi_{M}(m, V)=\left(d x^{1}, \ldots, d x^{n}\right)$. Moreover, for each integer $i=1, \ldots, n$, there is no difficulty to fix the point $x=x(m)$ and the tangent vector $v=d x(m)(V)$ but its $i$-th component, and calculate the partial derivative $\frac{\partial \psi}{\partial v^{i}}(x, v)$. The $\frac{\partial \psi}{\partial v^{i}}$ are the components of the differential $d(\psi(m,)$. obtained by fixing the base point $m$ and letting the tangent vector $V$ vary in $T_{m} M$. This differential is an element of the dual of $T_{m} M$. Evaluated at the vector $V$, it thus reads $\frac{\partial \psi}{\partial v^{i}}(x, v) d x^{i}$. It is called the partial differential of $\psi$ with respect to $V$ for fixed $m$, denoted below by $\left(\frac{\partial \psi}{\partial V}\right)_{m}$. In contrast to $d x^{i}$ and $\frac{\partial \psi}{\partial v^{i}}(x, v)$, the two other terms of the above formula, namely $d v^{i}$ and $\frac{\partial \psi}{\partial x^{i}}(x, v)$, do not transform properly under a change of charts (unless the atlas of $M$ is affine). They are not intrinsic. In order to deform the partial derivative $\frac{\partial \psi}{\partial x^{i}}(x, v)$ into an intrinsic expression, we need a tool to fix the tangent vector $V$ while the $i$-th coordinate of its base point $m$ varies near the value $x^{i}(m)$, all other coordinates being frozen. The Levi-Civita connection $\nabla$ is an appropriate tool. Using $\nabla$, we will keep the vector $V$ parallel to itself while transporting it along the path $t \mapsto\left(x^{1}(m), \ldots, x^{i-1}(m), x^{i}(m)+\right.$ $\left.t, x^{i+1}(m), \ldots, x^{n}(m)\right)$, for $t \in \mathbb{R}$ small. Doing so yields the intrinsic notion of partial differential of $\psi$ with respect to $m$ keeping $V$ parallel, denoted by $\left(\frac{\partial \psi}{\partial m}\right)_{V}^{\nabla}$, and defined at the point $(m, V)$ by

$$
\left(\frac{\partial \psi}{\partial m}\right)_{V}^{\nabla}(m, V)=\delta_{i}^{\nabla} \psi(x, v) d x^{i}, \text { with: } \delta_{i}^{\nabla}=\frac{\partial}{\partial x^{i}}-\Gamma_{i j}^{k}(x) v^{j} \frac{\partial}{\partial v^{k}},
$$

the $\Gamma_{i j}^{k}$ standing for the Christoffel symbols of the connection $\nabla$ in the chart $x$. Subtracting this expression from $d \psi$, we get

$$
d \psi-\left(\frac{\partial \psi}{\partial m}\right)_{V}^{\nabla}(m, V)=\frac{\partial \psi}{\partial v^{i}} d^{\nabla} v^{i}, \text { with: } d^{\nabla} v^{i}=d v^{i}+\Gamma_{k j}^{i}(x) v^{j} d x^{k} .
$$

Altogether, we have recast the differential of the function $\psi$ as follows:

$$
d \psi(m, V)=\delta_{i}^{\nabla} \psi(x, v) d x^{i}+\frac{\partial \psi}{\partial v^{i}}(x, v) d^{\nabla} v^{i} .
$$

We need some geometry to interpret further this expression, since the partial differential $\left(\frac{\partial \psi}{\partial V}\right)_{m}(m, V)$ was defined above as an element of $T_{m}^{*} M$ and its components $\frac{\partial \psi}{\partial v^{i}}$ are now associated with the covariant differentials $d^{\nabla} v^{i}$. The kernel $\mathcal{V}=\bigcap_{i=1}^{n} \operatorname{ker}\left(d x^{i}\right)$ of the differential $d \varpi_{M}$ is a subbundle of $T(T M) \rightarrow T M$ called the vertical subbundle and locally spanned by the $\frac{\partial}{\partial v^{i}}$ 's. The vector fields $\delta_{i}^{\nabla}, 1 \leqslant i \leqslant n$, span a subbundle $\mathcal{H}^{\nabla}$ of $T(T M) \rightarrow$ $T M$, called the horizontal subbundle, locally given by $\mathcal{H}^{\nabla}=\bigcap_{i=1}^{n} \operatorname{ker}\left(d^{\nabla} v^{i}\right)$ and such that the following splitting holds:

$$
T(T M)=\mathcal{H}^{\nabla} \oplus \mathcal{V}
$$


Moreover, the local fields of basis $\left\{\delta_{1}^{\nabla}, \ldots, \delta_{n}^{\nabla}, \frac{\partial}{\partial v^{1}}, \ldots, \frac{\partial}{\partial v^{n}}\right\}$ and cobasis $\left\{d x^{1}, \ldots, d x^{n}, d^{\nabla} v^{1}, \ldots, d^{\nabla} v^{n}\right\}$ are dual of each other. The horizontal projection $T(T M) \rightarrow \mathcal{H}^{\nabla}$ with kernel equal to $\mathcal{V}$ is given by $d m=d x^{i} \otimes \delta_{i}^{\nabla}$ and the vertical projection $T(T M) \rightarrow \mathcal{V}$ with kernel equal to $\mathcal{H}^{\nabla}$, by $d^{\nabla} V=d^{\nabla} v^{i} \otimes \frac{\partial}{\partial v^{i}}$. Every tangent vector $W=\xi^{i} \frac{\partial}{\partial x^{i}}+\eta^{i} \frac{\partial}{\partial v^{i}} \in T_{(m, V)}(T M)$ reads now uniquely as the sum of a horizontal vector $W_{\mathrm{h}}=d m(W)$ and a vertical one $W_{\mathrm{v}}=d^{\nabla} V(W)$, thus respectively equal to:

$$
W_{\mathrm{h}}=\xi^{i} \delta_{i}^{\nabla} \in \mathcal{H}_{(m, V)}^{\nabla}, W_{\mathrm{v}}=\left(\eta^{i}+\Gamma_{k j}^{i}(x) v^{j} \xi^{k}\right) \frac{\partial}{\partial v^{i}} \in \mathcal{V}_{(m, V)}
$$

Last but not least, we may identify the fiber $\mathcal{H}_{(m, V)}^{\nabla}$ with the tangent space $T_{m} M$ by means of the differential $d \varpi_{M}$ which induces the isomorphism from $\mathcal{H}_{(m, V)}^{\nabla}$ to $T_{m} M$ given by: $\delta_{i}^{\nabla} \rightarrow \frac{\partial}{\partial x^{i}}, i=1, \ldots, n$. We further identify $\mathcal{V}_{(m, V)}$ with $T_{m} M$ by the canonical isomorphism from $\mathcal{V}_{(m, V)}$ to $T_{m} M$ given by the affine structure of $T_{m} M$, or else, by: $\frac{\partial}{\partial v^{i}} \rightarrow \frac{\partial}{\partial x^{i}}, i=1, \ldots, n$. In this way, we may freely consider at $(m, V)$ the projections $d m$ and $d^{\nabla} V$, or else, the components $W_{\mathrm{h}}$ and $W_{\mathrm{v}}$ of $W \in T_{(m, V)}(T M)$, as valued in $T_{m} M$. Accordingly, we may view the partial differentials of $\psi$ at $(m, V)$ appearing in (2.2), that is $\left(\frac{\partial \psi}{\partial m}\right)_{V}^{\nabla}(m, V)$ and $\frac{\partial \psi}{\partial v^{i}}(x, v) d^{\nabla} v^{i}$, which are elements of the dual of $\mathcal{H}_{(m, V)}^{\nabla}$ and $\mathcal{V}_{(m, V)}$ respectively, as belonging to $T_{m}^{*} M$. Doing so, we may now write (2.2) as a global identity of the desired form, namely:

$$
d \psi(m, V)(W)=\left(\frac{\partial \psi}{\partial m}\right)_{V}^{\nabla}(m, V)\left(W_{\mathrm{h}}\right)+\left(\frac{\partial \psi}{\partial V}\right)_{m}(m, V)\left(W_{\mathrm{v}}\right) .
$$

Pulling the function $\psi$ back on $M$ by a vector field $U \in \Gamma(T M)$, thus setting $U^{*} \psi(m) \equiv \psi(m, U(m))$, we infer for every $m \in M, \xi \in T_{m} M$, the formula:

$$
\begin{aligned}
& d\left(U^{*} \psi\right)(m)(\xi)= \\
& \left(\frac{\partial \psi}{\partial m}\right)_{V}^{\nabla}(m, U(m))(\xi)+\left(\frac{\partial \psi}{\partial V}\right)_{m}(m, U(m))\left(\nabla_{\xi} U(m)\right),
\end{aligned}
$$

of constant use in the paper.

Remark 2.1. For later use, let us record here the existence of the so-called diagonal lift $g^{D}$ of the metric $g$ to the manifold $T M$, a Riemannian metric, often named after Sasaki (see [YI73] and references therein) and defined, using the preceding identifications, by:

$$
g_{(m, V)}^{D}(W, Z)=g_{m}\left(W_{\mathrm{h}}, Z_{\mathrm{h}}\right)+g_{m}\left(W_{\mathrm{v}}, Z_{\mathrm{v}}\right),
$$

for every point $(m, V) \in T M$ and every couple of vectors $W, Z$ in $T_{(m, V)}(T M)$. In particular, the factors $\mathcal{H}^{\nabla}$ and $\mathcal{V}$ of the splitting of $T(T M)$ are mutually orthogonal for the metric $g^{D}$. 


\subsubsection{Exponential map}

We can deal similarly with the exponential map exp : $T M \rightarrow M$, getting the identity:

$$
d \exp (m, V)(W)=\left(\frac{\partial \exp }{\partial m}\right)_{V}^{\nabla}(m, V)\left(W_{\mathrm{h}}\right)+\left(\frac{\partial \exp }{\partial V}\right)_{m}(m, V)\left(W_{\mathrm{v}}\right) .
$$

Here, the partial differentials of the exponential map at the point $(m, V)$ may be viewed as linear maps from $T_{m} M$ to $T_{p} M$, with $p=\exp _{m}(V)$, equal to the identity at $V=0$. As the point $(m, V)$ varies in $T M$, each of them gives rise to a section of the vector bundle $\operatorname{Mor}\left(\varpi_{M}^{*} T M, \exp ^{*} T M\right) \rightarrow T M$ of morphisms covering the identity, $\varpi_{M}^{*} T M \rightarrow T M$ (resp. $\exp ^{*} T M \rightarrow T M$ ) standing for the pullback of the tangent bundle $\varpi_{M}: T M \rightarrow M$ by the map $\varpi_{M}$ (resp. by the exponential map). Equivalently, we may view the maps $\left(\frac{\partial \exp }{\partial m}\right)_{V}^{\nabla}$ and $\left(\frac{\partial \exp }{\partial V}\right)_{m}$ as sections of the bundle $\varpi_{M}^{*} T^{*} M \otimes \exp ^{*} T M \rightarrow T M$.

Pulling the exponential map back to the base manifold $M$ by means of a vector field $U \in \Gamma(T M)$, we infer from (2.4) the further identity:

$$
\begin{aligned}
& d\left(U^{*} \exp \right)(m)(\xi)= \\
& \quad\left(\frac{\partial \exp }{\partial m}\right)_{V}^{\nabla}(m, U(m))(\xi)+\left(\frac{\partial \exp }{\partial V}\right)_{m}(m, U(m))\left(\nabla_{\xi} U(m)\right),
\end{aligned}
$$

where $U^{*} \exp (m) \equiv \exp _{m}(U(m))$.

\subsubsection{Jacobi endomorphism}

Let $\mathcal{N}_{\text {conj }}$ denote the open neighborhood of the zero section of $T M$ consisting of the points $(m, V) \in T M$ such that the linear map:

$$
\left(\frac{\partial \exp }{\partial V}\right)_{m}(m, V): T_{m} M \rightarrow T_{p} M, \text { with } p=\exp _{m}(V),
$$

remains an isomorphism. Given $(m, V) \in \mathcal{N}_{\text {conj }}$ and $\xi \in T_{m} M$, the equation:

$$
\left(\frac{\partial \exp }{\partial m}\right)_{V}^{\nabla}(m, V)(\xi)=\left(\frac{\partial \exp }{\partial V}\right)_{m}(m, V)(\nu),
$$

can be solved uniquely for $\nu \in T_{m} M$. It thus defines an endomorphism $\xi \in T_{m} M \rightarrow \mathcal{J}(m, V)(\xi)=\nu \in T_{m} M$, often called the Jacobi endomorphism of $T_{m} M$ at $(m, V)$. This endomorphism arises naturally in the description of the fibers of the exponential map, when the latter is restricted to $\mathcal{N}_{\text {conj }}$. Specifically, the tangent space to $\exp ^{-1}(p)$ at $(m, V) \in \mathcal{N}_{\text {conj }}$ is the subspace of $T_{(m, V)}(T M)$ given by the graph of the linear map from $\mathcal{H}_{(m, V)}^{\nabla}$ to $\mathcal{V}_{(m, V)}$ defined, with the usual identifications, by $-\mathcal{J}(m, V)$. The main properties of this endomorphism will be derived in section 2.3.2 below, using the Jacobi equation. Here, we just note that $\mathcal{J}(m, 0)$ must be equal to the identity. 
The map $(m, V) \in T M \rightarrow \mathcal{J}(m, V) \in \operatorname{End}\left(T_{m} M\right)$ is a section, over $\mathcal{N}_{\text {conj }}$, of the pullback bundle $\varpi_{M}^{*} \operatorname{End}(T M) \rightarrow T M$. Let us pause and explain a natural way of differentiating on $T M$ maps like the ones considered so far, namely $\left(\frac{\partial \exp }{\partial m}\right)_{V}^{\nabla},\left(\frac{\partial \exp }{\partial V}\right)_{m}$ and $\mathcal{J}$.

\subsubsection{Linear connections on vector bundles}

The material presented here for convenience is fairly well-known [PT88]. A linear connection $\nabla$ on a vector bundle $p: E \rightarrow Q$ over a closed manifold $Q$ is a map which associates, to each couple $((q, W), S)$ formed by a tangent vector $(q, W) \in T Q$ at a point $q \in Q$ and a section $S$ of the bundle $E \rightarrow Q$, a vector $\nabla_{W} S$ of the fiber $E_{q}=p^{-1}(q)$, in such a way that:

(i) given any integer $k$, for every $C^{k}$ vector field $W$ on the base manifold $Q$ and every $C^{k+1}$ section $S$ of the bundle $E \rightarrow Q$ :

(i.1) the section $\nabla_{W} S$ of that bundle is of class $C^{k}$ and the map $(W, S) \mapsto \nabla_{W} S$ so defined is smooth;

(i.2) the map $W \mapsto \nabla_{W} S$, defined by fixing the section $S$ and letting the vector field $W$ vary, is linear over the algebra $C^{k}(Q)$ of real functions of class $C^{k}$ on $Q$;

(ii) for each fixed tangent vector $(q, W)$, the map $S \mapsto \nabla_{W} S$ obtained by letting the section $S$ vary, is $\mathbb{R}$-linear and satisfies the chain rule, that is, the equation:

$$
\nabla_{W}(f S)=d f(W) S+f \nabla_{W} S,
$$

holds identically at the point $q$, for every real function $f \in C^{1}(Q)$.

Given a linear connection $\nabla$ on a vector bundle $E \rightarrow Q$, we can define new linear connections out of it by duality, tensor product and pullback. The dual connection $\nabla^{*}$ is the linear connection defined on the dual bundle $E^{*} \rightarrow Q$ by the equation:

$$
\nabla_{W}^{*} \ell(S)=d(\ell(S))(W)-\ell\left(\nabla_{W} S\right)
$$

valid for every vector field $W$ on $Q$ and sections $S$ of $E, \ell$ of $E^{*}$. The product connection $\nabla^{\otimes}$ on the tensor product $E^{*} \otimes E \rightarrow Q$, for instance, is given by:

$$
\nabla_{W}^{\otimes}(\ell \otimes S)=\left(\nabla^{*} \ell\right) \otimes S+\ell \otimes\left(\nabla_{W} S\right) .
$$

A similar definition can be given for more general tensor products, of course. In either case, we are guided by the chain rule for the defining equation and the axioms $(i)(i i)$ of a linear connection can readily be verified.

The pullback device is a bit trickier; it goes as follows. Given a map $\varphi: P \rightarrow Q$ between two closed manifolds and a vector bundle $E \rightarrow Q$ of 
rank $r$ endowed with a linear connection $\nabla$, we can define on the pullback bundle $\varphi^{*} E \rightarrow P$ a linear connection $\nabla^{\varphi}$ as follows. Fix a point $p_{0} \in P$ and a local frame field $q \in Q \rightarrow\left(S_{1}(q), \ldots, S_{r}(q)\right)$ spanning the fiber of E over the point $q$, for $q$ near the image point $q_{0}=\varphi\left(p_{0}\right)$. Such a frame field yields, for every section $\xi$ of the pullback bundle $\varphi^{*} E \rightarrow P$, local real functions $\xi^{1}, \ldots, \xi^{r}$ defined near the point $p_{0}$ in the source manifold $P$ and such that the section $\xi$ reads: $\xi(p)=\xi^{a}(p) S_{a}(q)$, with $q=\varphi(p)$. Now, for each vector field $V$ on $P$, a natural candidate for the covariant derivative $\nabla_{V}^{\varphi} \xi$ is given near the point $p_{0}$ by:

$$
\nabla_{V}^{\varphi} \xi(p)=d \xi^{a}(p)(V(p)) S_{a}(q)+\xi^{a}(p)\left(\nabla_{W} S_{a}\right)(q)
$$

with $q=\varphi(p)$ and $W=d \varphi(p)(V(p)) \in T_{q} Q$. The right-hand side of this equation turns out to be independent of the choice of the local frame $S_{i}$, hence of that of the point $p_{0}$ as well. So we have actually defined a global section $\nabla_{V}^{\varphi} \xi$ of the pullback bundle $\varphi^{*} E \rightarrow P$. Furthermore, one can readily check that $\nabla^{\varphi}$ fulfills the axioms $(i)(i i)$ of a linear connection on the vector bundle $\varphi^{*} E \rightarrow P$.

In case the source manifold $P$ is an interval (resp. the product of two intervals) of the real line, with generic point $t$ (resp. $(\tau, t)$ ), it is common to write $\frac{\nabla}{d t}\left(\right.$ resp. $\left.\left(\frac{\nabla}{\partial \tau}, \frac{\nabla}{\partial t}\right)\right)$ instead of $\nabla_{\frac{d}{d t}}^{\varphi}\left(\operatorname{resp} .\left(\nabla_{\frac{\partial}{\partial \tau}}^{\varphi}, \nabla_{\frac{\partial}{\partial t}}^{\varphi}\right)\right)$. If the map $\varphi: P \rightarrow Q$ is equal to, either $\varpi_{M}: T M \rightarrow M$ or $\exp : T M \rightarrow M$, and we take for the vector bundle $E \rightarrow Q$ the tangent bundle $T M \rightarrow M$ equipped with the Levi-Civita connection, we will omit the superscript $\varphi$ of $\nabla^{\varphi}$ and write $\nabla$ anytime no confusion may occur. Let us specify what are the coefficients of these connections in local charts. Sticking to the notations of section 2.1.1, we infer from (2.6) that they are given by:

$$
\begin{aligned}
& \nabla_{\delta_{i}^{\nabla}}^{\varpi_{M}}\left(\frac{\partial}{\partial x^{j}}\right)= \Gamma_{i j}^{k}(x) \frac{\partial}{\partial x^{k}}, \quad \nabla_{\frac{\partial}{\partial v^{i}}}^{\varpi_{M}}\left(\frac{\partial}{\partial x^{j}}\right)=0, \text { and: } \\
& \nabla_{\delta_{i}^{\nabla}}^{\exp }\left(\frac{\partial}{\partial \bar{x}^{j}}\right)=\left(\delta_{i}^{\nabla} E^{k}\right)(x, v) \bar{\Gamma}_{j k}^{\ell}(E(x, v)) \frac{\partial}{\partial \bar{x}^{\ell}} \\
& \nabla_{\frac{\partial}{\partial v^{i}}}^{\exp }\left(\frac{\partial}{\partial \bar{x}^{j}}\right)=\frac{\partial E^{k}}{\partial v^{i}}(x, v) \bar{\Gamma}_{j k}^{\ell}(E(x, v)) \frac{\partial}{\partial \bar{x}^{\ell}} .
\end{aligned}
$$

Here, we have used a chart $x$ (resp. $\bar{x}$ ) at the source point $m$ (resp. target point $\left.\bar{m}=\exp _{m}(V)\right)$, set $E(x, v)=\bar{x}\left(\exp _{m}(V)\right)$, where $x=x(m), v=$ $d x(m)(V)$, and denoted by $\Gamma_{i j}^{k}$ (resp. $\bar{\Gamma}_{i j}^{k}$ ) the Christoffel symbols of the Levi-Civita connection of $M$ in the chart $x$ (resp. $\bar{x}$ ). Combining the knowledge of these coefficients with the duality and tensor product devices described above, we are now in position to differentiate sections like 


$$
\begin{array}{r}
\left(\frac{\partial \exp }{\partial m}\right)_{V}^{\nabla},\left(\frac{\partial \exp }{\partial V}\right)_{m} \text { and } \mathcal{J} \text {. In the latter case, for instance, we find: } \\
\nabla_{\delta_{i}^{\nabla}} \mathcal{J}_{j}^{k}=\delta_{i}^{\nabla} \mathcal{J}_{j}^{k}+\Gamma_{i \ell}^{k}(x) \mathcal{J}_{j}^{\ell}-\Gamma_{i j}^{\ell}(x) \mathcal{J}_{\ell}^{k}=\nabla_{i} \mathcal{J}_{j}^{k}-\Gamma_{i h}^{\ell}(x) v^{h} \frac{\partial \mathcal{J}_{j}^{k}}{\partial v^{\ell}} \\
\text { and } \nabla_{\frac{\partial}{\partial v^{i}}} \mathcal{J}_{j}^{k}=\frac{\partial \mathcal{J}_{j}^{k}}{\partial v^{i}},
\end{array}
$$

with $\nabla_{i} \mathcal{J}_{j}^{k}=\frac{\partial \mathcal{J}_{j}^{k}}{\partial x^{i}}+\Gamma_{i \ell}^{k}(x) \mathcal{J}_{j}^{\ell}-\Gamma_{i j}^{\ell}(x) \mathcal{J}_{\ell}^{k}$. Let us emphasize that the local functions $\nabla_{i} \mathcal{J}_{j}^{k}$ are not the components of a tensor, unlike the functions $\nabla_{\delta_{i}} \mathcal{J}_{j}^{k}$. Nevertheless, it will be convenient to use them in some calculations.

Remark 2.2 (general erratum). In his previous works, anytime the author had to differentiate twice a section of a vector bundle over $T M$ (obtained by pulling back a tensor bundle over $M$ ) for the purpose of a second order estimate performed in a normal chart of $M$, he made a meaningless use of the original Levi-Civita connection $\nabla$, instead of using the appropriate pullback connection. However, his estimates are still valid. Indeed, every rectification of this mistake yields only first order terms with curvature coefficients, which are inessential for the estimate. Section 6.2 of the present paper illustrates the correct way to proceed.

\section{$2.2 C^{\ell}$ norms}

We need to define $C^{\ell}$ norms of real functions, or of sections of pullback tensor bundles, defined on (the closure of) some smooth bounded domain $\Omega$ of $T M$. For real functions, we do it using the Sasaki metric $g^{D}$ (see Remark 2.1 ) and its Levi-Civita connection. For sections of $\varpi_{M}^{*} T M$, we first note that this vector bundle inherits an obvious avatar of the metric $g$. Moreover, the connection $\nabla^{\varpi_{M}}$ is compatible with the latter. Combining the preceding tools, we can define the Banach space $C^{\ell}\left(\bar{\Omega}, \varpi_{M}^{*} T M\right)$. A similar definition can be given for the space $C^{\ell}\left(\bar{\Omega}, \varpi_{M}^{*} \operatorname{End}(T M)\right)$.

\section{$2.3 \quad$ Jacobi fields and applications}

\subsubsection{Jacobi equation}

Given $\left(m_{0}, V_{0}\right) \in T M$, we set $\mathcal{X}_{\gamma_{0}}$ for the vector space of all vector fields $t \mapsto Y(t) \in T_{\gamma_{0}(t)} M$ along the geodesic path $t \mapsto \gamma_{0}(t)=\exp _{m_{0}}\left(t V_{0}\right) \in M$, and let $\mathfrak{J}_{\gamma_{0}} \in \operatorname{End}\left(\mathcal{X}_{\gamma_{0}}\right)$ denote the Jacobi operator along $\gamma_{0}$. Recall that the latter reads:

$$
Y \mapsto \mathfrak{J}_{\gamma_{0}}(Y)(t)=\frac{\nabla^{2} Y}{d t^{2}}+R_{\gamma_{0}(t)}\left(Y, \dot{\gamma}_{0}(t)\right) \dot{\gamma}_{0}(t),
$$


where $R$ stands for the curvature (or Riemann) tensor ${ }^{4}$, of the Levi-Civita connection $\nabla$ and the dot, as usual, for the time derivative. If we change the initial time from 0 to $\tau$, setting $\gamma_{\tau}(t)=\exp _{\gamma_{0}(\tau)}\left(t \dot{\gamma}_{0}(\tau)\right)$, we have $\gamma_{\tau}(t-\tau) \equiv$ $\gamma_{0}(t)$, hence $\dot{\gamma}_{\tau}(t-\tau) \equiv \dot{\gamma}_{0}(t)$ and:

$$
R_{\gamma_{0}(t)}\left(., \dot{\gamma}_{0}(t)\right) \dot{\gamma}_{0}(t) \equiv R_{\gamma_{\tau}(\theta)}\left(., \dot{\gamma}_{\tau}(\theta)\right) \dot{\gamma}_{\tau}(\theta) \text { with } \theta=t-\tau .
$$

We infer that, for every $Y_{0} \in \mathcal{X}_{\gamma_{0}}$, the vector field $Y_{\tau} \in \mathcal{X}_{\gamma_{\tau}}$ defined by $Y_{\tau}(t-\tau)=Y_{0}(t)$ satisfies identically $\mathfrak{J}_{\gamma_{\tau}}\left(Y_{\tau}\right)(t-\tau) \equiv \mathfrak{J}_{\gamma_{0}}\left(Y_{0}\right)(t)$.

If a vector field $Y_{0} \in \mathcal{X}_{\gamma_{0}}$ satisfies the Jacobi equation $\mathfrak{J}_{\gamma_{0}} Y_{0}=0$, it is called a Jacobi field. Note that: $Y_{0} \in \operatorname{ker} \mathfrak{J}_{\gamma_{0}} \Longleftrightarrow Y_{\tau} \in \operatorname{ker} \mathfrak{J}_{\gamma_{\tau}}$. Regarding the special way in which Jacobi fields arise, we refer the reader to Riemannian geometry textbooks [CE08, pp.14-15] [dC92, Chap.5]. Here, we would like to point out that $Y_{0} \in \mathcal{X}_{\gamma_{0}}$ is a Jacobi field if and only it satisfies the following initial value identity:

$$
Y_{0}(t) \equiv\left(\frac{\partial \exp }{\partial m}\right)_{V}^{\nabla}\left(m_{0}, t V_{0}\right)\left(Y_{0}(0)\right)+t\left(\frac{\partial \exp }{\partial V}\right)_{m}\left(m_{0}, t V_{0}\right)\left(\frac{\nabla Y_{0}}{d t}(0)\right) .
$$

Indeed, this identity expresses exactly what geometers prove about Jacobi fields, for instance in the books just quoted.

Remark 2.3. As is well-known and easy to check, the velocity field $\dot{\gamma}_{0}$ is a Jacobi field along the geodesic path $\gamma_{0}$, and so is the field $t \mapsto t \dot{\gamma}_{0}(t)$. From (2.8), we see that the former is equal to $\left(\frac{\partial \exp }{\partial m}\right)_{V}^{\nabla}\left(m_{0}, t V_{0}\right)\left(V_{0}\right)$ and the latter, to $t\left(\frac{\partial \exp }{\partial V}\right)_{m}\left(m_{0}, t V_{0}\right)\left(V_{0}\right)$. Besides, for every Jacobi field $Y_{0}$, one readily verifies that the scalar product $g_{\gamma_{0}(t)}\left(Y_{0}(t), \dot{\gamma}_{0}(t)\right)$ must be an affine function of $t$. If $V_{0} \neq 0$, it follows that the linear maps $\left(\frac{\partial \exp }{\partial m}\right)_{V}^{\nabla}\left(m_{0}, t V_{0}\right)$ and $\left(\frac{\partial \exp }{\partial V}\right)_{m}\left(m_{0}, t V_{0}\right)$ send $V_{0}^{\perp}$ to $\dot{\gamma}_{0}(t)^{\perp}$.

Let us describe some applications of the identity (2.8) to be used in the paper.

\subsubsection{Applications}

Explicit calculations For each point $(m, V) \in T M$, the endomorphism of $T_{m} M$ given by: $Y \mapsto R_{m}(Y, V) V$, often called the Jacobi curvature endomorphism along the vector $V$, is symmetric. If $U$ is a nontrivial eigenvector orthogonal to $V$, the corresponding eigenvalue is equal to $|U|^{2}|V|^{2} K(p), p$ standing for the tangent 2-plane at $m$ defined by the vectors $U$ and $V$, and $K$, for the sectional curvature of the manifold $M$ in the direction of that 2-plane.

\footnotetext{
${ }^{4}$ with the sign convention given by: $R(U, V) W=\nabla_{U} \nabla_{V} W-\nabla_{V} \nabla_{U} W-\nabla_{[U, V]} W$, for every triple of vector fields $U, V, W$
} 
If $V_{0} \neq 0$ and the manifold $M$ is Riemannian locally symmetric, that is, if the Riemann tensor is parallel, it is possible to take along the geodesic $\gamma_{0}$ a parallel orthonormal frame field $\left(e_{1}(t), \ldots, e_{n}(t)\right)$, with $\dot{\gamma}_{0}(t)=\left|V_{0}\right| e_{n}(t)$, in which the matrix of the Jacobi curvature endomorphism along $\dot{\gamma}_{0}(t)$ remains diagonal and constant as $t$ varies. Setting $Y_{0}(t)=\sum_{i=1}^{n} Y_{0}^{i}(t) e_{i}(t)$ and $R_{m_{0}}\left(e_{\alpha}(0), V_{0}\right) V_{0}=\left|V_{0}\right|^{2} \kappa_{\alpha} e_{\alpha}(0), \alpha=1, \ldots, n-1$, the Jacobi equation $\mathfrak{J}_{\gamma_{0}}\left(Y_{0}\right)=0$ becomes: $\frac{d^{2} Y_{0}^{\alpha}}{d t^{2}}+\left|V_{0}\right|^{2} \kappa_{\alpha} Y_{0}^{\alpha}=0, \alpha<n$, and $\frac{d^{2} Y_{0}^{n}}{d t^{2}}=0$. So the Cauchy problem can be solved explicitly. In particular, we can calculate the matrices of the maps $\left(\frac{\partial \exp }{\partial m}\right)_{V}^{\nabla}\left(m_{0}, V_{0}\right)$ and $\left(\frac{\partial \exp }{\partial V}\right)_{m}\left(m_{0}, V_{0}\right)$, as diagonal matrices with the $\alpha$-th entries depending only on $\left|V_{0}\right|$ and the sectional curvature $\kappa_{\alpha}$, and with the $n$-th entries both equal to 1 .

Inhomogeneous Jacobi equations Dropping the local symmetry assumption on $M$, let us apply the identity (2.8) to solve in $\mathcal{X}_{\gamma_{0}}$ the inhomogeneous Cauchy problem:

$$
\mathfrak{J}_{\gamma_{0}}(Y)=U, Y(0)=\xi, \frac{\nabla Y}{d t}(0)=\nu,
$$

where $\xi, \nu$ in $T_{m} M$ and $U \in \mathcal{X}_{\gamma_{0}}$ are given. Specifically, writing the equation $\mathfrak{J}_{\gamma_{0}}(Y)=U$ as a first order system in $\left(\begin{array}{l}Y \\ Z\end{array}\right)$ with $Z=\frac{\nabla Y}{d t}$, we note that the resolvent $\mathcal{R}(t, \tau)$ of the homogeneous system is given by:

$$
\left(\begin{array}{cc}
\left(\frac{\partial \exp }{\partial m}\right)_{V}^{\nabla}\left(\gamma_{0}(\tau),(t-\tau) \dot{\gamma}_{0}(\tau)\right) & (t-\tau)\left(\frac{\partial \exp }{\partial V}\right)_{m}\left(\gamma_{0}(\tau),(t-\tau) \dot{\gamma}_{0}(\tau)\right) \\
\frac{\nabla}{\partial t}\left[\left(\frac{\partial \exp }{\partial m}\right)_{V}^{\nabla}\left(\gamma_{0}(\tau),(t-\tau) \dot{\gamma}_{0}(\tau)\right)\right] & \frac{\nabla}{\partial t}\left[(t-\tau)\left(\frac{\partial \exp }{\partial V}\right)_{m}\left(\gamma_{0}(\tau),(t-\tau) \dot{\gamma}_{0}(\tau)\right)\right]
\end{array}\right) .
$$

For instance, from $(2.8)$, we have $W(t)=\mathcal{R}(t, 0) W(0)$, with $W=\left(\begin{array}{c}Y_{0} \\ \frac{\nabla Y_{0}}{d t}\end{array}\right)$. The map $t \mapsto \mathcal{R}(t, \tau)$ satisfies the initial condition $\mathcal{R}(\tau, \tau)=\mathrm{I} d$ and the composition identity: $\mathcal{R}(t, \theta) \mathcal{R}(\theta, \tau) \equiv \mathcal{R}(t, \tau)$. Now, the method of variation of constants [Car67, Har02] yields for the solution of (2.9) the representation formula:

$$
Y(t)=Y_{0}(t)+\int_{0}^{t}(t-\tau)\left(\frac{\partial \exp }{\partial V}\right)_{m}\left(\gamma_{0}(\tau),(t-\tau) \dot{\gamma}_{0}(\tau)\right)(U(\tau)) d \tau
$$

where $Y_{0}$ is the Jacobi field given by first component of $W(t)=\mathcal{R}(t, 0)\left(\begin{array}{l}\xi \\ \nu\end{array}\right)$.

Estimates From the Jacobi equation combined with Schwarz inequality, we can find a constant $C>0$ depending only on $n$ and $\max _{\mathrm{Gr}_{2}(M)}|K|$, still setting $\mathrm{Gr}_{2}(M)$ for the Grassmann bundle of tangent 2-planes on $M$, such that the energy of every Jacobi field $Y_{0} \in \mathcal{X}_{\gamma_{0}}$ satisfies the differential inequality: $\frac{d}{d t}\left(\left|Y_{0}(t)\right|^{2}+\left|\frac{\nabla Y_{0}}{d t}(t)\right|^{2}\right) \leqslant C\left(1+\left|V_{0}\right|^{2}\right)\left(\left|Y_{0}(t)\right|^{2}+\left|\frac{\nabla Y_{0}}{d t}(t)\right|^{2}\right)$, hence the estimate:

$$
\left|Y_{0}(t)\right|^{2}+\left|\frac{\nabla Y_{0}}{d t}(t)\right|^{2} \leqslant e^{C\left(1+\left|V_{0}\right|^{2}\right) t}\left(\left|Y_{0}(0)\right|^{2}+\left|\frac{\nabla Y_{0}}{d t}(0)\right|^{2}\right)
$$


In particular, with the convention that the norm of the identity is equal to $\sqrt{n}$, we infer from $(2.8)$ that the norms of the linear maps $\left(\frac{\partial \exp }{\partial m}\right)_{V}^{\nabla}\left(m_{0}, V_{0}\right)$ and $\left(\frac{\partial \exp }{\partial V}\right)_{m}\left(m_{0}, V_{0}\right)$ are bounded above by $e^{C\left(1+\left|V_{0}\right|^{2}\right) / 2} \sqrt{n}$.

If the eigenvalues of the curvature endomorphism along the geodesic segment $t \in[0,1] \rightarrow \gamma_{0}(t) \in M$ are nonnegative and if that segment contains no conjugate point, the Rauch comparison theorem [dC92, p.215] [CE08, p.29] yields a sharper bound, namely: $t\left|\left(\frac{\partial \exp }{\partial V}\right)_{m}\left(m_{0}, t V_{0}\right)\right| \leqslant t \sqrt{n}$. If so, we infer from (2.10) the estimate:

$$
\max _{[0, t]}\left|Y(t)-Y_{0}(t)\right| \leqslant \frac{t^{2}}{2} \sqrt{n} \max _{[0, t]}|U(t)|,
$$

for the solution of the initial value problem (2.9).

Main properties of the Jacobi endomorphism We infer at once from Remark 2.3 (read at $t=1$ ) the:

Proposition 2.1. The identity $\mathcal{J}\left(m_{0}, V_{0}\right) V_{0}=V_{0}$ holds at every tangent

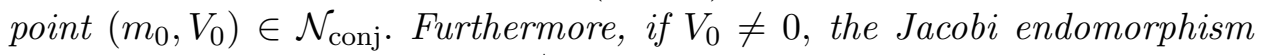
$\mathcal{J}\left(m_{0}, V_{0}\right)$ maps the subspace $V_{0}^{\perp}$ to itself.

Let us fix a point $\left(m_{0}, V_{0}\right) \in \mathcal{N}_{\text {conj }}$, set $p_{0}=\exp _{m_{0}}\left(V_{0}\right)$ and consider the equation $\exp _{m}(V)=p_{0}$ for $(m, V) \in T M$ close to $\left(m_{0}, V_{0}\right)$. Since $\left(\frac{\partial \exp }{\partial V}\right)_{m}\left(m_{0}, V_{0}\right): T_{m_{0}} M \rightarrow T_{p_{0}} M$ is an isomorphism, we may apply the implicit function theorem at $\left(m_{0}, V_{0}\right)$ to that equation. It yields a local vector field $\widehat{V}$, equal to $V_{0}$ at the point $m_{0}$, and such that the identity $\exp _{m}(\widehat{V}(m)) \equiv p_{0}$ holds for $m$ close enough to $m_{0}$. We take $m$ so close to $m_{0}$ that the graph of the vector field $\widehat{V}$ remains inside $\mathcal{N}_{\text {conj }}$. By differentiating the preceding identity, the reader will find (exercise) that the derivative of the local vector field $\widehat{V}$ is given by:

$$
\nabla_{\xi} \widehat{V}(m)=-\mathcal{J}(m, \widehat{V}(m))(\xi),
$$

for every vector $\xi \in T_{m} M$. We further study the vector field $\widehat{V}$ itself, by introducing the local function $\hat{c}(m)=\frac{1}{2}|\widehat{V}(m)|^{2}$. The latter represents half the squared length of the geodesic path joining the point $m$ to the fixed endpoint $p_{0}$, with initial velocity $\widehat{V}(m)$ close to $V_{0}$. From $(2.13)$, the derivative of that local squared length function is given by: $d \hat{c}(m)(\xi)=$ $g_{m}(-\widehat{V}(m), \mathcal{J}(m, \widehat{V}(m))(\xi))$. From Proposition 2.1, the right-hand side of this equation is equal to $g_{m}(-\widehat{V}(m), \xi)$. Since the vector $\xi \in T_{m} M$ is arbitrary, we have proved the local identity:

$$
\widehat{V}(m)=-\operatorname{grad} \hat{c}(m) .
$$


Plugging the latter in (2.13), we further get:

$$
\mathcal{J}(m, \widehat{V}(m)) \equiv \nabla \operatorname{grad} \hat{c}(m) .
$$

In particular, letting $m=m_{0}$, we infer the

Proposition 2.2. The Jacobi endomorphism $\mathcal{J}\left(m_{0}, V_{0}\right): T_{m_{0}} M \rightarrow T_{m_{0}} M$ is symmetric, for every $\left(m_{0}, V_{0}\right) \in \mathcal{N}_{\text {conj }}$.

Let us now focus on the behavior of the eigenvalues of the symmetric endomorphism $\mathcal{J}^{\perp}\left(m_{0}, V_{0}\right)$ induced on $V_{0}^{\perp}$ by $\mathcal{J}\left(m_{0}, V_{0}\right)$. The following proposition extends Lemma 3.12 and Proposition 2.5 of [CMS01], from $\mathcal{N}_{\text {cut }}$ (defined below) to $\mathcal{N}_{\text {conj }}$, with a simpler proof based on the Index Lemma.

Proposition 2.3. For every $\left(m_{0}, V_{0}\right) \in \mathcal{N}_{\text {conj }}, V_{0} \neq 0$, setting $\mathcal{J}^{\perp}\left(m_{0}, V_{0}\right)$ for the endomorphism of $V_{0}^{\perp}$ induced by $\mathcal{J}\left(m_{0}, V_{0}\right)$, the following upper bounds hold in the sense of symmetric endomorphisms:

(i) $\mathcal{J}^{\perp}\left(m_{0}, V_{0}\right) \leqslant \mathrm{Id}$, if the Jacobi curvature endomorphism along $\dot{\gamma}_{0}(t)$ is nonnegative for all $t \in[0,1]$;

(ii) $\mathcal{J}^{\perp}\left(m_{0}, V_{0}\right) \leqslant\left(\left|V_{0}\right| \sqrt{\kappa} \operatorname{coth}\left|V_{0}\right| \sqrt{\kappa}\right) \mathrm{Id}$, if $-\kappa<0$ stands for a lower bound on the eigenvalues of the Jacobi curvature endomorphism along $\dot{\gamma}_{0}(t)$, valid for all $t \in[0,1]$.

Furthermore, for every converging sequence $\left(V_{k}\right)_{k \in \mathbb{N}}$ of $T_{m_{0}} M$ such that $\left(m_{0}, V_{k}\right)$ lies in $\mathcal{N}_{\text {conj }}$ for each $k \in \mathbb{N}$ and the limit point $\left(m_{0}, V_{\infty}\right)$ lies on the boundary of $\mathcal{N}_{\text {conj }}$, the sequence of symmetric endomorphisms $\left(\mathcal{J}\left(m_{0}, V_{k}\right)\right)_{k \in \mathbb{N}}$ is unbounded below.

Proof. We denote henceforth by $S_{m}$ the sectional curvature tensor (or covariant Riemann tensor) of the manifold at the point $m$. Following [CMS01], we use the index form of the geodesic $t \in[0,1] \rightarrow \gamma_{0}(t)=$ $\exp _{m_{0}}\left(t v_{0}\right) \in M$, namely the bilinear form $I_{\gamma_{0}}$ given by:

$$
I_{\gamma_{0}}(Y, Z)=\int_{0}^{1}\left(g_{\gamma_{0}(t)}\left(\frac{\nabla Y}{d t}, \frac{\nabla Z}{d t}\right)-S_{\gamma_{0}(t)}\left(Y(t), \dot{\gamma}_{0}(t), Z(t), \dot{\gamma}_{0}(t)\right)\right) d t
$$

which acts on the subspace $\mathcal{X}_{\gamma_{0}}^{\perp}$ of $\mathcal{X}_{\gamma_{0}}$ consisting of vector fields everywhere orthogonal to $\gamma_{0}$. We fix a unit vector $\xi \in V_{0}^{\perp}$ and consider the Jacobi field $J_{\xi}$ equal to:

$$
J_{\xi}(t)=\left(\frac{\partial \exp }{\partial m}\right)_{V}^{\nabla}\left(m_{0}, t V_{0}\right)(\xi)-t\left(\frac{\partial \exp }{\partial V}\right)_{m}\left(m_{0}, t V_{0}\right)\left(\mathcal{J}\left(m_{0}, V_{0}\right)(\xi)\right) .
$$

By reading (2.13) at $m=m_{0}$, we know that this field vanishes at $t=1$. The combination of Remark 2.3 with Proposition 2.1 further shows that it 
belongs to $\mathcal{X}_{\gamma_{0}}^{\perp}$. Moreover, integration by parts yields for the field $J_{\xi}$ the identity:

$$
I_{\gamma_{0}}\left(J_{\xi}, J_{\xi}\right)=g_{m_{0}}\left(\mathcal{J}\left(m_{0}, V_{0}\right)(\xi), \xi\right) .
$$

For every vector field $Z \in \mathcal{X}_{\gamma_{0}}^{\perp}$ with the same endpoint values as $J_{\xi}$, namely $Z(0)=\xi, Z(1)=0$, the Index Lemma [Aub98, p.19] [dC92, pp.212-215] [CE08, pp.24-25], applied after a time reversion, provides the fundamental inequality:

$$
I_{\gamma_{0}}\left(J_{\xi}, J_{\xi}\right) \leqslant I_{\gamma_{0}}(Z, Z) .
$$

Here, time reversion means that $\gamma_{0}$ is replaced by the geodesic $t \in[0,1] \rightarrow$ $\widetilde{\gamma}_{0}(t)=\gamma_{0}(1-t)$ and each vector field $Y \in \mathcal{X}_{\gamma_{0}}^{\perp}$, by the vector field $\widetilde{Y} \in \underset{\mathcal{X}}{\stackrel{\perp}{\gamma_{0}}}$ given by $\widetilde{Y}(t)=Y(1-t)$. It is straightforward to verify that $Y$ is a Jacobi field along $\gamma_{0}$ if and only if $\widetilde{Y}$ is so along $\widetilde{\gamma}_{0}$.

Under assumption $(i)$, we apply $(2.16)$ with $Z(t)=(1-t) \xi_{\gamma_{0}}(t)$, where $\xi_{\gamma_{0}}(t)$ denotes the parallel transport of $\xi$ from $m_{0}$ to $\gamma_{0}(t)$ along $\tau \in[0, t] \rightarrow$ $\gamma_{0}(\tau)$. The desired conclusion comes from the obvious bound $I_{\gamma_{0}}(Z, Z) \leqslant 1$. If we assume $(i i)$ instead, the following upper bound holds:

$$
\left.I_{\gamma_{0}}(Z, Z) \leqslant \int_{0}^{1}\left(\left|\frac{\nabla Z}{d t}\right|_{\gamma_{0}(t)}^{2}+\kappa\left|V_{0}\right|^{2}|Z|_{\gamma_{0}(t)}^{2}\right)\right) d t
$$

for every $Z \in \mathcal{X}_{\gamma_{0}}^{\perp}$. The right-hand side corresponds formally to the index form that would occur if the Jacobi curvature endomorphism field along the geodesic $\gamma_{0}$ had $n-1$ eigenvalues constant equal to $-\kappa$. If it were so, on the one hand, the Jacobi field $J_{\xi}$ would formally coincide with the field:

$$
Z(t)=\left(\cosh \left(t\left|V_{0}\right| \sqrt{\kappa}\right)-\operatorname{coth}\left(\left|V_{0}\right| \sqrt{\kappa}\right) \sinh \left(t\left|V_{0}\right| \sqrt{\kappa}\right)\right) \xi_{\gamma_{0}}(t)
$$

on the other hand, the eigenvalues of the formal analogue of $\mathcal{J}^{\perp}\left(m_{0}, V_{0}\right)$ would be constant equal to $\left(\left|V_{0}\right| \sqrt{\kappa} \operatorname{coth}\left|V_{0}\right| \sqrt{\kappa}\right)$. Plugging this expression of $Z$ in the above right-hand integral, we thus know from (2.15) that we must get the desired conclusion (exercise: compute the integral). The first part of Proposition 2.3 is established.

In order to prove its second part, following [CMS01], we fix a small real $\alpha>0$ and argue by contradiction, assuming the existence of a sequence $\left(V_{k}\right)_{k \in \mathbb{N}}$, as stated, and of a constant $C>0$ such that the lower bound: $g_{m_{0}}\left(\mathcal{J}\left(m_{0}, V_{k}\right)(\xi), \xi\right) \geqslant-C$ holds, for every integer $k$ and unit vector $\xi \in$ $T_{m_{0}} M$. Setting $\gamma_{k}(t)=\exp _{m_{0}}\left(t V_{k}\right)$, we infer from (2.15) and (2.16) that the bound: $I_{\gamma_{k}}\left(Z_{k}, Z_{k}\right) \geqslant-C \alpha^{2}$, holds uniformly with respect to $k \in \mathbb{N}$ and to the vector fields $Z_{k} \in \mathcal{X}_{\gamma_{k}}^{\perp}$ satisfying: $Z_{k}(0)=\alpha \xi, Z_{k}(1)=0$. Letting $k$ tend to $\infty$, we get:

$$
I_{\gamma_{\infty}}(Z, Z) \geqslant-C \alpha^{2},
$$

where $\gamma_{\infty}(t)=\exp _{m_{0}}\left(t V_{\infty}\right)$, a lower bound valid for every vector field $Z \in$ $\mathcal{X}_{\gamma_{\infty}}^{\perp}$ satisfying: $Z(0)=\alpha \xi, Z(1)=0$. We now mimick [CMS01] for the rest 
of the proof. From our assumption on the limit point $\left(m_{0}, V_{\infty}\right)$, we may take $\xi$ in the kernel of the map $\left(\frac{\partial \exp }{\partial V}\right)_{m}\left(m_{0}, V_{\infty}\right)$. Fixing $\xi$ so, we choose the vector field $Z$ equal to: $Z(t)=Y_{\xi}(t)+\alpha(1-t) \xi_{\gamma_{\infty}}(t)$, with $Y_{\xi}(t)=$ $t\left(\frac{\partial \exp }{\partial V}\right)_{m}\left(m_{0}, t V_{\infty}\right)(\xi)$. The latter is such that the integral $I_{\gamma_{\infty}}\left(Y_{\xi}, Y_{\xi}\right)$ must vanish [CE08, p.21]. Besides, we readily find $I_{\gamma_{\infty}}\left(Y_{\xi},(1-t) \xi_{\gamma_{\infty}}\right)=-1$. It follows that (2.17), read with our choice of $Z$, implies the inequality:

$$
-2 \alpha+\left(I_{\gamma_{\infty}}\left((1-t) \xi_{\gamma_{\infty}},(1-t) \xi_{\gamma_{\infty}}\right)+C\right) \alpha^{2} \geqslant 0
$$

which is absurd, provided the real $\alpha>0$ is taken small enough

\subsection{Jacobians}

\subsubsection{Calibrated determinants}

Let $X$ be a nonoriented real vector space of dimension $n$ and $\pm \omega$, a nonzero $n$-covector on $X$ defined up to sign. Let $\left(X^{\prime}, \pm \omega^{\prime}\right)$ and $\left(X^{\prime \prime}, \pm \omega^{\prime \prime}\right)$ be similarly defined with the same integer $n$. The calibrated determinant of a linear map $L: X \rightarrow X^{\prime}$ is given by:

$$
\operatorname{det}_{|\omega|,\left|\omega^{\prime}\right|}(L)=\left|\frac{\omega^{\prime}\left(L e_{1}, \ldots, L e_{n}\right)}{\omega\left(e_{1}, \ldots, e_{n}\right)}\right|
$$

where $\left\{e_{1}, \ldots, e_{n}\right\}$ stands for a basis of $X$. This definition does not depend on the choice of that basis, nor on the sign \pm chosen before $\omega$ and $\omega^{\prime}$. If $X=X^{\prime}$ and $|\omega|=\left|\omega^{\prime}\right|$, the definition becomes independent of the choice of $\pm \omega$ itself and reduces, up to the absolute value, to the well-known notion of determinant of an endomorphism. Like the latter, the calibrated determinant has a nice multiplication property, namely:

$$
\operatorname{det}_{|\omega|,\left|\omega^{\prime \prime}\right|}\left(L^{\prime} \circ L\right) \equiv \operatorname{det}_{|\omega|,\left|\omega^{\prime}\right|}(L) \operatorname{det}_{\left|\omega^{\prime}\right|,\left|\omega^{\prime \prime}\right|}\left(L^{\prime}\right),
$$

for every couple of linear maps $L: X \rightarrow X^{\prime}$ and $L^{\prime}: X^{\prime} \rightarrow X^{\prime \prime}$. Indeed, both sides vanish if $L$ is not an isomorphism and, if it is, we just have to write:

$$
\left|\frac{\omega^{\prime \prime}\left(\left(L^{\prime} \circ L\right) e_{1}, \ldots,\left(L^{\prime} \circ L\right) e_{n}\right)}{\omega\left(e_{1}, \ldots, e_{n}\right)}\right| \equiv\left|\frac{\omega^{\prime \prime}\left(L^{\prime} v_{1}, \ldots, L^{\prime} v_{n}\right)}{\omega^{\prime}\left(v_{1}, \ldots, v_{n}\right)}\right| \times\left|\frac{\omega^{\prime}\left(L e_{1}, \ldots, L e_{n}\right)}{\omega\left(e_{1}, \ldots, e_{n}\right)}\right|,
$$

with $v_{i}=L e_{i}$, to make (2.19) obvious.

Objects like $(X, \pm \omega)$ arise naturally in Riemannian geometry. Indeed, at each point $m$ of our closed nonoriented Riemannian manifold $(M, g)$, the tangent space $T_{m} M$ is endowed with a nonzero $n$-covector defined up to sign, namely the restriction to $T_{m} M$ of the volume form $\omega_{g}$. The latter is defined by $\omega_{g}(m)= \pm \theta^{1} \wedge \ldots \wedge \theta^{n}$, where $\left\{\theta^{1}, \ldots, \theta^{n}\right\}$ stands for an orthonormal basis of $T_{m}^{*} M$. 


\subsubsection{Exponential map at a point}

Fixing the point $m \in M$ and letting the vector $V$ vary in $T_{m} M$, consider the family of linear maps $\left(\frac{\partial \exp }{\partial V}\right)_{m}(m, V): T_{m} M \rightarrow T_{p} M$, with $p=\exp _{m}(V)$. The Jacobian of the map $\exp _{m}: T_{m} M \rightarrow \mathbb{R}$ at $V \in T_{m} M$ is defined by:

$$
\operatorname{Jac}\left(\exp _{m}\right)(V)=\operatorname{det}_{\left|\omega_{g}(m)\right|,\left|\omega_{g}(p)\right|}\left(\left(\frac{\partial \exp }{\partial V}\right)_{m}(m, V)\right) .
$$

Letting now $m \in M$ vary, we get a smooth nonnegative function on $T M$, equal to 1 along the zero section and nonvanishing on $\mathcal{N}_{\text {conj }}$. Let us pause and illustrate briefly the use of that function.

Henceforward, we denote by $d V_{g}$ (or just $d V$ when not confusing) the volume measure of the metric $g$ and by $\mathcal{N}_{\text {cut }}$, the open neighborhood of the zero section of $T M$ consisting of the points $(m, V) \in T M$ such that the geodesic segment $t \in[0,1] \rightarrow \exp _{m}(t V) \in M$ contains no cut point. As is well-known, the inclusion $\mathcal{N}_{\text {cut }} \subseteq \mathcal{N}_{\text {conj }}$ holds; moreover, the map $\exp _{m}$ sends the tangential domain $\mathcal{N}_{\text {cut }}(m)=\mathcal{N}_{\text {cut }} \cap T_{m} M$ diffeomorphically onto the open subset of full measure $M \backslash \operatorname{Cut}(m)$, where $\operatorname{Cut}(m)$ denotes the cut locus of the point $m$ [dC92, CE08].

The function $(m, V) \mapsto \operatorname{Jac}\left(\exp _{m}\right)(V)$ occurs anytime one computes an integral like $\int_{M} h(p) d V_{g}(p), h \in L^{1}\left(M, d V_{g}\right)$. Using the change of variables $\bar{m}=\exp _{m}(V), V \in \mathcal{N}_{\text {cut }}$, the integral becomes:

$$
\int_{M} h(\bar{m}) d V_{g}(\bar{m})=\int_{\mathcal{N}_{\text {cut }}(m)}\left(h \circ \exp _{m}\right) \operatorname{Jac}\left(\exp _{m}\right)(V) d v_{g_{m}}(V),
$$

where $d v_{g_{m}}$ stands for the Lebesgue measure on $T_{m} M$ canonically associated to the $n$-covector $\omega_{g}(m)$. If the manifold $M$ is Riemannian locally symmetric, as pointed out in the first paragraph of Section 2.3.2, we can calculate explicitly the expression of the Jacobi fields along the geodesic $t \mapsto \exp _{m}(t V)$. The reader can do the exercise or find the solution in [Bes78, p.82] in the positively curved case. In the notations of Section 7.1 , we get in that case:

$$
\operatorname{Jac}\left(\exp _{m}\right)(V)=\left(\frac{\sin |V|}{|V|}\right)^{\nu}\left(\frac{\sin \frac{|V|}{2}}{\frac{|V|}{2}}\right)^{n-\nu-1} .
$$

This expression factors as a function of the sole radial variable $r=|V|$, decreasing from 1 to 0 on $[0, \pi)$. The computation of the above integral is now tractable. Taking for instance $h=1$ on a geodesic ball of radius $r<\pi$, and zero elsewhere, we can compute in this way the volume of that ball.

Dropping local symmetry and letting the point $(m, V)$ vary in $T M$, the expression of the function $(m, V) \mapsto \operatorname{Jac}\left(\exp _{m}\right)(V)$ itself is given in any couple of charts by:

$$
\operatorname{Jac}\left(\exp _{m}\right)(V)=\sqrt{\frac{\operatorname{det}\left(g_{\overline{i j}}(E(x, v))\right)}{\operatorname{det}\left(g_{i j}(x)\right)}}\left|\operatorname{det}\left(\frac{\partial E^{\bar{i}}}{\partial v^{j}}(x, v)\right)\right| .
$$


Here, we have used a chart $x$ (resp. $\bar{x}$ ) at the source point $m$ (resp. target point $\left.\bar{m}=\exp _{m}(V)\right)$, set $E(x, v)=\bar{x}\left(\exp _{m}(V)\right)$, where $x=x(m), v=$ $d x(m)(V)$, and denoted by $g_{i j}(x)$ (resp. $\left.g_{i \bar{j}}(\bar{x})\right)$ the local components of the metric tensor.

\subsubsection{Maps between Riemannian manifolds}

Let $\phi: M \rightarrow M^{\prime}$ be a map ranging in another unoriented Riemannian $n$-manifold (with metric $g^{\prime}$ ). For every point $m \in M$, setting for short $\omega_{m}=\omega_{g}(m), \omega_{m}^{\prime}=\omega_{g^{\prime}}(\phi(m))$, the Jacobian of the map $\phi$ is the nonnegative function $\operatorname{Jac}(\phi)$ defined on the manifold $M$ by:

$$
\operatorname{Jac}(\phi)(m)=\operatorname{det}_{\left|\omega_{m}\right|,\left|\omega_{m}^{\prime}\right|}(d \phi(m)) .
$$

If $\psi: M^{\prime} \rightarrow M^{\prime \prime}$ is a further map between unoriented Riemannian manifolds, the following multiplication property:

$$
\operatorname{Jac}(\psi \circ \phi) \equiv \operatorname{Jac}(\phi) .(\operatorname{Jac}(\psi) \circ \phi),
$$

can be infered from (2.19) in a straightforward way. In case $\phi: M \rightarrow M^{\prime}$ is a diffeomorphism, the pullback tensor $\phi^{*} g^{\prime}$ is a Riemannian metric on $M$ and the identity $d V_{\phi^{*} g^{\prime}}=\operatorname{Jac}(\phi) d V_{g}$ holds between the volume measures of the metrics $g$ and $\phi^{*} g^{\prime}$, due to (2.21). So the change of variable formula $\int_{M^{\prime}} h^{\prime} d V_{g^{\prime}}=\int_{M}\left(h^{\prime} \circ \phi\right) d V_{\phi^{*} g^{\prime}}$, valid for every function $h^{\prime} \in L^{1}\left(M^{\prime}, d V_{g^{\prime}}\right)$, becomes:

$$
\int_{M^{\prime}} h^{\prime} d V_{g^{\prime}}=\int_{M}\left(h^{\prime} \circ \phi\right) \operatorname{Jac}(\phi) d V_{g} .
$$

Besides, the volume measure of $\phi^{*} g^{\prime}$ coincides with the pushforward of the measure $d V_{g^{\prime}}$ by the inverse mapping $\phi^{-1}: M^{\prime} \rightarrow M$, that is:

$$
d V_{\phi^{*} g^{\prime}}=\left(\phi^{-1}\right)_{\#} d V_{g^{\prime}} \text {, or else, }\left(\phi^{-1}\right)_{\#} d V_{g^{\prime}}=\operatorname{Jac}(\phi) d V_{g} .
$$

Indeed, on the one hand, setting $h^{\prime}=h \circ \phi^{-1}$, with $h \in L^{1}\left(M, d V_{g}\right)$, the above change of variable formula yields the identity $\int_{M^{\prime}}\left(h \circ \phi^{-1}\right) d V_{g^{\prime}}=\int_{M} h d V_{\phi^{*} g^{\prime}}$. On the other hand, we have $\int_{M^{\prime}}\left(h \circ \phi^{-1}\right) d V_{g^{\prime}}=\int_{M} h\left(\left(\phi^{-1}\right)_{\#} d V_{g^{\prime}}\right)$, by the very definition of the pushforward measure.

Finally, the expression of the function $m \mapsto \operatorname{Jac}(\phi)(m)$ in any couple of charts is given by:

$$
\operatorname{Jac}(\phi)(m)=\sqrt{\frac{\operatorname{det}\left(g_{\overline{i j}}(\phi(x))\right)}{\operatorname{det}\left(g_{i j}(x)\right)}}\left|\operatorname{det}\left(\frac{\partial \phi^{\bar{i}}}{\partial x^{j}}(x)\right)\right| .
$$

Here, we have used a chart $x$ (resp. $\bar{x}$ ) at the source point $m$ (resp. target point $\bar{m}=\phi(m))$, set abusively $\phi(x)=\bar{x}(\phi(m))$, where $x=x(m)$, and denoted by $g_{i j}(x)$ (resp. $\left.g_{\overline{i j}}(\bar{x})\right)$ the local components of the metric tensor. 


\subsubsection{Maps factorizing through nonconjugating vector fields}

In the sequel, it will be convenient to call nonconjugating, a vector field $U$ on $M$ which ranges in $\mathcal{N}_{\text {conj }}$ (see section 2.1.3). Abusively, one may call so the map $\left(U^{*} \exp \right): M \rightarrow M$, as well. Similarly, we call the vector field $U$ (and the map $U^{*} \exp$ ) noncutting, provided $U$ ranges in $\mathcal{N}_{\text {cut }} \subseteq \mathcal{N}_{\text {conj }}$ (see section 2.4.2). Finally, we call $U$ (and $U^{*}$ exp, as well) strictly minimizing, if for every point $m \in M$, there exists a unique minimizing geodesic joining $m$ to its image point $\exp _{m}(U(m))$.

Let $U$ be a nonconjugating vector field on $M$. Since $U$ is so, we may recast the expression (2.5) of the differential of the map ( $\left.U^{*} \exp \right)$ as follows:

$$
d\left(U^{*} \exp \right)(m)(\xi)=\left(\frac{\partial \exp }{\partial V}\right)_{m}(m, U(m)) \circ\left(\mathcal{J}(m, U(m))(\xi)+\nabla_{\xi} U(m)\right),
$$

for every $m \in M, \xi \in T_{m} M$. From the multiplication property (2.19), we infer for the Jacobian of that map the expression:

$$
\begin{aligned}
\operatorname{Jac}\left(U^{*} \exp \right)(m)=\operatorname{Jac}\left(\exp _{m}\right) & (U(m)) \\
\times & |\operatorname{det}(\mathcal{J}(m, U(m))(.)+\nabla \cdot U(m))| .
\end{aligned}
$$

A similar expression was derived differently in [Cab97, pp.632-635], under the more restrictive assumption that $U$ is noncutting.

\section{Foundations of the PDE approach}

Back to the Monge problem described in the introduction, and sticking to the notations adopted there, let us investigate the smoothness issue raised by McCann's theorem. If, for every couple of $C^{k, \alpha}$ positive probability measures $\left(\mu_{0}, \mu_{1}\right)$, the potential function $u\left(\mu_{0}, \mu_{1}\right)$ is $C^{k+2, \alpha}$, given such a couple, the optimal maps $\operatorname{Mc}\left(\mu_{0}, \mu_{1}\right)$ and $\operatorname{Mc}\left(\mu_{1}, \mu_{0}\right)$ are $C^{k+1, \alpha}$. From the last part of Theorem 1.1, these maps are now inverse of each other at every point of the manifold $M$, hence diffeomorphisms of $M$. It follows that the potential function $u\left(\mu_{0}, \mu_{1}\right)$ must be a classical solution of the Monge-Ampère equation (1.2). This is what we infer from McCann's theorem; but we do not want to rely on that theorem.

In this section, forgetting the map $\operatorname{Mc}\left(\mu_{1}, \mu_{0}\right)$ and allowing provisionally the given densities $\rho_{0}, \rho_{1}$ to be merely continuous on the manifold, we will establish the following result:

Theorem 3.1. Let $M$ be a closed Riemannian manifold and $\left(\mu_{0}, \mu_{1}\right), a$ couple of continuous positive probability measures on $M . A C^{2}$ function $u: M \rightarrow \mathbb{R}$ is such that the gradient-mapping $G_{u}=\exp (\operatorname{grad} u): M \rightarrow M$ pushes $\mu_{0}$ to $\mu_{1}$ if and only if $u$ is a classical solution of the Monge-Ampère equation (1.2). Moreover, if so, the map $G_{u}$ must be a noncutting diffeomorphism and the function $u$ must be c-convex. 
This statement yields the founding ansatz of the PDE approach, namely:

Corollary 3.1. Let $M$ be a closed Riemannian manifold and $\left(\mu_{0}, \mu_{1}\right)$, a couple of $C^{k, \alpha}$ positive probability measures on $M$. McCann's potential function $u\left(\mu_{0}, \mu_{1}\right)$ is $C^{k+2, \alpha}$ if and only if the Monge-Ampère equation (1.2) admits a $C^{k+2, \alpha}$ solution.

Proof of the Corollary. By the preceding theorem, if $u\left(\mu_{0}, \mu_{1}\right)$ is $C^{k+2, \alpha}$, it must solve (1.2). Conversely, if $u$ is a $C^{k+2, \alpha}$ solution of (1.2), it must be $c$-convex and the map $G_{u}$, be a diffeomorphism, hence push $\mu_{0}$ to $\mu_{1}$. In the latter case, McCann's uniqueness theorem [McC01, Theorem 8] implies that the maps $G_{u}$ and $\operatorname{Mc}\left(\mu_{0}, \mu_{1}\right)$ coincide. So do the gradients of the functions $u$ and $u\left(\mu_{0}, \mu_{1}\right)$, because the map $G_{u}$ is noncutting. Therefore McCann's potential function $u\left(\mu_{0}, \mu_{1}\right)$ must be $C^{k+2, \alpha}$

The rest of the section is devoted to the proof of Theorem 3.1. We will proceed stepwise, starting with a series of auxiliary results of independent interest, then combining them all to derive the theorem itself. The first lemma was the object of [Fig12].

Lemma 3.1 (Figalli). Let $\phi: M \rightarrow M$ be a $C^{1}$ map pushing $\mu_{0}$ to $\mu_{1}$. The Jacobian of $\phi$ must be bounded below by $\frac{\min _{M} \rho_{0}}{\max _{M} \rho_{1}}>0$.

Proof. Claim 1: the open subset $\{\operatorname{Jac}(\phi)>0\}$ is nonempty.

Indeed, if it were empty, the image of $\phi$ would have zero volume measure, by Sard's theorem [Mil97]. For every Borel subset $B$ of $M \backslash \phi(M)$, we would get: $\mu_{1}(B)=\mu_{0}\left(\phi^{-1}(B)\right)=0$, contradicting the positivity assumption made on the target measure $\mu_{1}$.

Claim 2: the subsets $\{\operatorname{Jac}(\phi)>0\}$ and $\left\{\operatorname{Jac}(\phi) \geqslant \frac{\min _{M} \rho_{0}}{\max _{M} \rho_{1}}\right\}$ coincide.

Fix a point $m_{0} \in\{\operatorname{Jac}(\phi)>0\}$. The inverse function theorem, applied to the map $\phi$ at $m_{0}$, yields a neighborhood $\widetilde{\mathcal{U}}_{0}$ of $m_{0}$ such that $\phi$ induces a diffeomorphism $\widetilde{\phi}: \widetilde{\mathcal{U}}_{0} \rightarrow \phi\left(\widetilde{\mathcal{U}}_{0}\right)$. Setting $\widetilde{\mu}_{0}$ for the restriction of the measure $\mu_{0}$ to the neighborhood $\widetilde{\mathcal{U}}_{0}$, we consider the measure $\widetilde{\mu}_{1}=\widetilde{\phi}_{\#} \widetilde{\mu}_{0}$ on $\phi\left(\widetilde{\mathcal{U}}_{0}\right)$. Since $\widetilde{\phi}$ is a diffeomorphism, the measure $\widetilde{\mu}_{1}$ must be smooth positive; we set $\widetilde{\rho}_{1}=\frac{d \widetilde{\mu}_{1}}{d V}$. We observe that $\widetilde{\rho}_{1} \leqslant \rho_{1}$, due to the assumption $\phi_{\#} \mu_{0}=\mu_{1}$ and the obvious inclusion $\widetilde{\phi}^{-1}(B) \subset \phi^{-1}(B)$ holding for every Borel subset $B$ of $\phi\left(\widetilde{\mathcal{U}}_{0}\right)$. Furthermore, we may write for $\widetilde{\phi}$ the change of variables formula:

$$
\widetilde{\rho}_{1}(\widetilde{\phi}(m)) \operatorname{Jac}(\widetilde{\phi})(m)=\rho_{0}(m),
$$

valid at every point $m \in \widetilde{\mathcal{U}}_{0}$. From $\widetilde{\rho}_{1} \leqslant \rho_{1}$, we get the lower bound: $\operatorname{Jac}(\widetilde{\phi})(m) \geqslant \frac{\rho_{0}(m)}{\rho_{1}(\widetilde{\phi}(m))}$. Since Jac is a local operator, we may drop the tilda over $\phi$ in that inequality. We infer that $\widetilde{\mathcal{U}}_{0}$ is contained in $\{\operatorname{Jac}(\phi) \geqslant$ 
$\left.\frac{\min _{M} \rho_{0}}{\max _{M} \rho_{1}}\right\}$. In particular, Claim 2 is proved.

Conclusion: From the preceding claims, we know that the open subset $\{\operatorname{Jac}(\phi)>0\}$ is nonempty and closed. It must coincide with the manifold $M$, since the latter is connected. So the lemma holds on the whole of $M$, due to Claim 2

Lemma 3.2. If a $C^{2}$ function $u: M \rightarrow \mathbb{R}$ is such that the Jacobian of its gradient-mapping $G_{u}$ nowhere vanishes on $M$, the map $G_{u}: M \rightarrow M$ must be a diffeomorphism.

Proof. By the inverse function theorem [Lan02], the map $G_{u}$ must be a local diffeomorphism. Its image is thus open. By compactness of the manifold $M$ and continuity of the map $G_{u}$, the image $G_{u}(M)$ must also be closed. It follows that $G_{u}(M)=M$, since the manifold is connected. So $G_{u}: M \rightarrow M$ is a covering map. If the manifold is simply connected, we are done: the covering map $G_{u}$ must be 1-sheeted, hence a diffeomorphism. If not, let $p: \widetilde{M} \rightarrow M$ denote the universal covering space of $M$. We endow the manifold $\widetilde{M}$ with the pull-back Riemannian metric $\widetilde{g}$. The projection $p$ is now an isometry and so is every element $\gamma: \widetilde{M} \rightarrow \widetilde{M}$ of the covering transformation group $\widetilde{\Gamma}$ (isomorphic to $\pi_{1}(M)$ ). Let us consider the function $\widetilde{u}: \widetilde{M} \rightarrow \mathbb{R}$ given by: $\widetilde{u}=u \circ p$. Set $\widetilde{G}: \widetilde{M} \rightarrow \widetilde{M}$ for the corresponding gradient-mapping. From the invariance of the function $\widetilde{u}$ under the action of the group $\widetilde{\Gamma}$, it is not difficult to figure out that $\gamma \circ \widetilde{G}=\widetilde{G} \circ \gamma$, for every deck transformation $\gamma \in \widetilde{\Gamma}$. Furthermore, the naturality of gradient-mappings yields the identity: $p \circ \widetilde{G}=G_{u} \circ p$. In particular, the Jacobian of the map $\widetilde{G}$ nowhere vanishes on $\widetilde{M}$ therefore, by our previous argument, this map must be a diffeomorphism. We will use this result to prove that the covering map $G_{u}$ must be 1-sheeted. Arguing by contradiction, let us suppose the existence of a point $\bar{m} \in M$ and two distinct points $m \neq m^{\prime}$ in $M$, such that (dropping the subscript $u$ ): $G(m)=G\left(m^{\prime}\right)=\bar{m}$. Pick a point $\widetilde{m}$ in the fiber $p^{-1}(m)$ and another one $\widetilde{m}^{\prime}$ in $p^{-1}\left(m^{\prime}\right)$. Their images $\widetilde{G}(\widetilde{m})$ and $\widetilde{G}\left(\widetilde{m}^{\prime}\right)$ both lie in the fiber $p^{-1}(\bar{m})$, so there exists a covering transformation $\gamma: \widetilde{M} \rightarrow \widetilde{M}$ such that $\widetilde{G}(\widetilde{m})=\gamma \circ \widetilde{G}\left(\widetilde{m}^{\prime}\right)$. But $\gamma \circ \widetilde{G}\left(\widetilde{m}^{\prime}\right)=\widetilde{G}\left(\gamma\left(\widetilde{m}^{\prime}\right)\right)$ and the map $\widetilde{G}: \widetilde{M} \rightarrow \widetilde{M}$ is one-to-one, therefore $\widetilde{m}=\gamma\left(\widetilde{m}^{\prime}\right)$ hence $m=m^{\prime}$, which is absurd

Lemma 3.3. If a $C^{2}$ function $u: M \rightarrow \mathbb{R}$ is such that $\operatorname{Jac}\left(G_{u}\right)$ nowhere vanishes, it must be c-convex.

Proof. For every $C^{1}$ function $u: M \rightarrow \mathbb{R}$ and every point $\bar{m} \in M$, the function $p \in M \rightarrow-c(p, \bar{m})-u(p) \in \mathbb{R}$ is continuous. It thus assumes, at some point $p=m \in M$, a global maximum which coincides with the value $u^{c}(\bar{m})$ taken at the point $\bar{m}$ by the $c$-supremal convolution ${ }^{5}$ of $u$. For

\footnotetext{
${ }^{5}$ defined in the introduction
} 
$p \in M$ close to the point $m$, we have: $c(p, \bar{m})-c(m, \bar{m}) \geqslant u(m)-u(p)$, hence the function $p \mapsto c(p, \bar{m})$ is subdifferentiable at the point $m$. Being also superdifferentiable [McC01], it must be differentiable at $m$, from what we may conclude that $\partial c(m, \bar{m})=d u(m)$ hence also, by the proof given on top p.599 of [McC01], that $\bar{m}=G_{u}(m)$. Here, in the spirit of a convenient notation used in [KM10, KM12], we have set $d c=\partial c+\bar{\partial} c$ for the splitting of the differential of the two-point cost function $c$ into $\partial c(m, \bar{m})$ and $\bar{\partial} c(m, \bar{m})$, respectively covectors at the points $m$ and $\bar{m}$.

Now, if $u$ is $C^{2}$ and $\operatorname{Jac}\left(G_{u}\right)$ does not vanish, the map $G_{u}$ must be a diffeomorphism by the preceding lemma. We thus get: $m=G_{u}^{-1}(\bar{m})$, with $m$ a differentiability point of the function $c(., \bar{m})$. Letting the point $\bar{m}$ vary in the manifold, the function $\bar{m} \mapsto u^{c}(\bar{m})$ is now given by: $u^{c}(\bar{m})=$ $-c\left(G_{u}^{-1}(\bar{m}), \bar{m}\right)-u\left(G_{u}^{-1}(\bar{m})\right)$. It is thus differentiable.

Playing with $u^{c}$ the game just played with $u$, we fix the point $m \in M$ and consider the function $q \in M \rightarrow-c(m, q)-u^{c}(q) \in \mathbb{R}$. It assumes at some point $q=\bar{m}$ a global maximum equal to $\left(u^{c}\right)^{c}(m)$. Recalling the general inequality $u^{c}(\bar{m}) \geqslant-c(m, \bar{m})-u(m)$, we see that $\left(u^{c}\right)^{c}(m) \leqslant u(m)$. If this inequality were strict, the definition of $\left(u^{c}\right)^{c}$ would imply that $u(m)>$ $-c(m, q)-u^{c}(q)$, for every point $q \in M$. But this is false at $q=G_{u}(m)$. Therefore $\left(u^{c}\right)^{c}(m)=u(m)$ and since the point $m \in M$ is arbitrary, we conclude that $u$ is, indeed, $c$-convex

Lemma 3.4. If a $C^{2}$ function $u: M \rightarrow \mathbb{R}$ is such that $\operatorname{Jac}\left(G_{u}\right)$ nowhere vanishes, the map $G_{u}: M \rightarrow M$ must be strictly minimizing.

Proof. If $\operatorname{Jac}\left(G_{u}\right)$ does not vanish, we know from the preceding proof that the function $q \in M \rightarrow-c(m, q)-u^{c}(q) \in \mathbb{R}$ must assume a global maximum at the point $q=G_{u}(m)$. In the neighborhood of that point, we thus have: $c(m, q)-c\left(m, G_{u}(m) \geqslant u^{c}\left(G_{u}(m)\right)-u^{c}(q)\right.$, which shows that the function $q \mapsto c(m, q)$ is subdifferentiable, hence differentiable [McC01], at the very point $q=G_{u}(m)$. This result implies (see top p.232 of [CMS01]) the nonexistence of two distinct minimizing geodesics going from $m$ to $G_{u}(m)$, as claimed

Proposition 3.1. If a $C^{2}$ function $u: M \rightarrow \mathbb{R}$ is such that $\operatorname{Jac}\left(G_{u}\right)$ does not vanish, the map $\exp _{m}: T_{m} M \rightarrow M$ must have full rank at the tangent vector $\operatorname{grad} u(m)$ and the symmetric endomorphism $\mathcal{H} u(m)$ defined by:

$$
\mathcal{H} u(m)=\mathcal{J}(m, \operatorname{grad} u(m))+\nabla \operatorname{grad} u(m),
$$

must be strictly positive, for every point $m \in M$.

Consistently with the term used in [DG10, p.69], we call the endomorphism $\mathcal{H} u(m): T_{m} M \rightarrow T_{m} M$ the $c$-Hessian endomorphism of the function $u$ at the point $m$. 
Proof. Fix a point $m_{0} \in M$ where the function $u$ assumes a local minimum. The conclusions of the lemma are satisfied at the point $m_{0}$. Let $S_{u}\left(m_{0}\right)$ be the connected component of the open subset $S_{u} \subset M$, given by:

$$
S_{u}=\left\{m \in M, \operatorname{Jac}\left(\exp _{m}\right)(\operatorname{grad} u(m))>0 \text { and } \mathcal{H} u(m)>0\right\},
$$

containing the point $m_{0}$. It suffices to prove that $S_{u}\left(m_{0}\right)$ is closed. Indeed, if it is so, the lemma holds because the manifold $M$ itself is connected. Accordingly, let us consider a sequence $\left(m_{i}\right)_{i \in \mathbb{N}}$ of $S_{u}\left(m_{0}\right)$ with limit point $m_{\infty}=\lim _{i \rightarrow \infty} m_{i} \in M$. For fixed $i \in \mathbb{N}$, Lemma 3.4 and the definition of the subset $S_{u}$ imply that the point $\left(m_{i}, \operatorname{grad} u\left(m_{i}\right)\right)$ lies in $\mathcal{N}_{\text {cut }}$. Besides, from the positivity of $\mathcal{H} u\left(m_{i}\right)$, we have: $\mathcal{J}\left(m_{i}, \operatorname{grad} u\left(m_{i}\right)\right) \geqslant-C_{2} \operatorname{Id}_{m_{i}}$, where the real $C_{2} \geqslant 0$ stands for the largest eigenvalue on $M$ of the Hessian endomorphism field $\nabla \operatorname{grad} u$. Letting $i$ tend to infinity, we get at the limit point $m_{\infty}$ three inequalitites, namely: $\operatorname{Jac}\left(\exp _{m_{\infty}}\right)\left(\operatorname{grad} u\left(m_{\infty}\right)\right) \geqslant 0, \mathcal{H} u\left(m_{\infty}\right) \geqslant$ 0 , and $\mathcal{J}\left(m_{\infty}, \operatorname{grad} u\left(m_{\infty}\right)\right) \geqslant-C_{2} \operatorname{Id}_{m_{\infty}}$. From the latter combined with the last part of Proposition 2.3, we infer that the point $\left(m_{\infty}, \operatorname{grad} u\left(m_{\infty}\right)\right)$

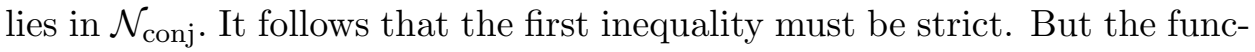
tion $\operatorname{Jac}\left(G_{u}\right)$ does not vanish on $M$. Therefore, reading the equation (2.25) at $m=m_{\infty}$ with the vector field $U=\operatorname{grad} u$, we conclude that the middle inequality, that is $\mathcal{H} u\left(m_{\infty}\right) \geqslant 0$, must also be strict

Lemma 3.5. If a $C^{2}$ function $u: M \rightarrow \mathbb{R}$ is such that $\operatorname{Jac}\left(G_{u}\right)$ nowhere vanishes, the map $G_{u}: M \rightarrow M$ must be noncutting.

Proof. For every point $m \in M$, we know by Lemma 3.4 that the image point $G_{u}(m)$ can be joined to $m$ by a unique minimizing geodesic, namely $t \in[0,1] \rightarrow G_{t u}(m) \in M$. By the first part of Proposition 3.1, we further know that this geodesic contains no conjugate point. Therefore, indeed, it contains no cut point

Proof of Theorem 3.1 With the preceding lemmas at hand, the proof of the theorem is not difficult. Still, it should be provided since it contains a major step of our method, namely the reduction of the Monge problem to the classical solvability of the Monge-Ampère equation (1.2).

Proof of the first part of Theorem 3.1. Let the function $u$ lie in $C^{2}(M, \mathbb{R})$ and be such that the gradient-mapping $G_{u}$ pushes $\mu_{0}$ to $\mu_{1}$. The combination of Lemmas 3.1 and 3.2 implies that the map $G_{u}: M \rightarrow M$ is a diffeomorphism. Applying, on the one hand the change of variable formula (2.23) read with $M^{\prime}=M, \phi=G_{u}, h^{\prime}=h \rho_{1}, h \in L^{1}(M, d V)$, on the other hand the pushing formula (1.1) read with $\phi=G_{u}$, we see that the equation:

$$
\int_{M}\left(h \circ G_{u}\right)\left(\rho_{1} \circ G_{u}\right) \operatorname{Jac}\left(G_{u}\right) d V=\int_{M}\left(h \circ G_{u}\right) \rho_{0} d V,
$$

holds for every function $h \in L^{1}(M, d V)$. We infer from this identity that the $C^{2}$ function $u$ must satisfy (1.2) pointwise. Conversely, if the latter 
conclusion is now our assumption, on the one hand the identity (3.1) holds, on the other hand the map $G_{u}$ must be a diffeomorphism due to Lemma 3.2. But if $G_{u}$ is so, the left-hand side of (3.1) can be equated to $\int_{M} h d \mu_{1}$ by means of the change of variable formula $(2.23)$ read as we did above. It follows that the map $G_{u}$ pushes the measure $\mu_{0}$ to the measure $\mu_{1}$. The first part of the theorem is proved.

The proof of the second part of Theorem 3.1 is now straightforward. Indeed, we just proved in either case that the gradient-mapping $G_{u}$ must be a diffeomorphism. So we may apply to it Lemmas 3.3 and 3.5 and get the desired conclusion

Remark 3.1. The uniqueness, up to addition of a constant, of the $C^{2}$ solution of the Monge-Ampère equation (1.2), is another aspect of the PDE approach, consistent with the uniqueness of the optimal map $\operatorname{Mc}\left(\mu_{0}, \mu_{1}\right)$ proven in [McC01]. It will be established in Section 4.3 (Proposition 4.2).

\section{Continuity method}

Recall that we have fixed in the introduction a smoothness degree, namely a couple $(k, \alpha) \in \mathbb{N} \times(0,1)$, with $k \geqslant 2$. Accordingly, we focus on the construction of a $C^{k+2, \alpha}$ solution $u: M \rightarrow \mathbb{R}$ of the Monge-Ampère equation (1.2) posed on a closed Riemannian manifold $M$, with volume measure $d V$, endowed with a couple of $C^{k, \alpha}$ positive probability densities $\left(\rho_{0}, \rho_{1}\right)$. By Corollary 3.1, this construction is equivalent to showing that McCann's optimal potential function $u\left(\rho_{0} d V, \rho_{1} d V\right)$ is $C^{k+2, \alpha}$. We will perform it by means of the continuity method.

Specifically, we will argue by connectedness of the subset $\mathcal{T} \subset[0,1]$ consisting of the all values of the parameter $t \in[0,1]$ for which one can find a $C^{k+2, \alpha}$ solution $u_{t}$ of the deformed equation:

$$
\rho_{t}\left(G_{u}(m)\right) \operatorname{Jac}\left(G_{u}\right)(m)=\rho_{0}(m),
$$

where $\rho_{t}$ stands for the $C^{k, \alpha}$ positive probability density given by $\rho_{t}=$ $t \rho_{1}+(1-t) \rho_{0}$. For $t=1$, the latter coincides with the original equation (1.2) to be solved. For $t=0$, it has the obvious solution $u_{0}=0$, so the subset $\mathcal{T}$ is nonempty and we will prove successively that it is relatively open in $[0,1]$ and closed.

\subsection{Openess part of the method}

The idea of that part is to use an implicit function theorem argument. Let us describe the related functional analytic setting. We say that a $C^{2}$ function $u: M \rightarrow \mathbb{R}$ is admissible, if $\operatorname{Jac}\left(G_{u}\right)$ nowhere vanishes. For each integer $\ell$ and real number $a$, we set $C_{a}^{\ell, \alpha}$ for the affine Banach space of $C^{\ell, \alpha}$ real functions with average on $M$ equal to $a$ and denote by $\mathcal{A}^{k+2, \alpha}$ the 
open subset of all admissible functions in $C_{0}^{k+2, \alpha}$. By Lemma 3.2 and the observation that, if $u$ solves (4.1), so does $u$ +constant, we may take the solutions of (4.1) in $\mathcal{A}^{k+2, \alpha}$ with no loss of generality. Besides, we consider the open subset $P_{1}^{k, \alpha}$ of all positive functions in $C_{1}^{k, \alpha}$. We note that the tangent bundles to $\mathcal{A}^{k+2, \alpha}$ and to $P_{1}^{k, \alpha}$ are both trivial, with constant fiber consisting of the Banach space $C_{0}^{\ell, \alpha}$, respectively with $\ell=k+2$ and $\ell=k$. Finally, we let $\Phi:[0,1] \times \mathcal{A}^{k+2, \alpha} \rightarrow C^{k, \alpha}(M, \mathbb{R})$ be the operator given by: $\Phi(t, u)(m)=\rho_{t}\left(G_{u}(m)\right) \operatorname{Jac}\left(G_{u}\right)(m)$, for every point $m \in M$.

Lemma 4.1. The operator $\Phi$ ranges in $P_{1}^{k, \alpha}$. Moreover, for fixed $t \in[0,1]$, the differential $d \Phi(t,$.$) is an isomorphism from C_{0}^{k+2, \alpha}$ to $C_{0}^{k, \alpha}$, at every function $u \in \mathcal{A}^{k+2, \alpha}$.

Proof. Fix a couple $(t, u) \in[0,1] \times \mathcal{A}^{k+2, \alpha}$. The $C^{k, \alpha}$ function $\Phi(t, u)$ must be positive on the manifold because so are the density $\rho_{t}$ and the Jacobian of the map $G_{u}$. Indeed, the latter cannot vanish because $G_{u}$ is a diffeomorphism. For the same reason, the density $\rho_{u}=\frac{d \mu_{u}}{d V}$ of the probability measure $\mu_{u}$ defined by $\mu_{u}=\left(G_{u}\right)_{\#} \mu_{0}$ must belong to $P_{1}^{k, \alpha}$. The identity $\Phi(t, u) \equiv\left(\left(\frac{\rho_{t}}{\rho_{u}}\right) \circ G_{u}\right) \rho_{0}$ is thus satisfied on the manifold. Integrating it over $M$ and applying the pushing condition $\left(G_{u}\right)_{\#} \mu_{0}=\mu_{u}$, we get: $\int_{M} \Phi(t, u) d V=\int_{M} \frac{\rho_{t}}{\rho_{u}} d \mu_{u}=\int_{m} \rho_{t} d V=1$. Therefore the map $\Phi$ ranges in $P_{1}^{k, \alpha}$, as claimed. Let us pause for a remark of constant use in the sequel.

Remark 4.1 (actual expression of the Monge-Ampère operator). From the combination of Proposition 3.1 with the expression of $\operatorname{Jac}\left(G_{u}\right)$ given by (2.25) read at the vector field $U=\operatorname{grad} u$, we infer that the operator $\Phi$ is actually given by:

$$
\Phi(t, u)(m)=\rho_{t}\left(G_{u}(m)\right) \operatorname{Jac}\left(\exp _{m}\right)(\operatorname{grad} u(m)) \operatorname{det}(\mathcal{H} u(m)),
$$

at every admissible function $u \in \mathcal{A}^{k+2, \alpha}$ and point $(t, m) \in[0,1] \times M$. Here, the $c$-Hessian endomorphism field $\mathcal{H} u$, which was defined in Proposition 3.1 by $\mathcal{H} u(m)=\mathcal{J}(m, \operatorname{grad} u(m))+\nabla \operatorname{grad} u(m)$, is symmetric and must stay positive everywhere on the manifold.

Back to the interrupted proof, we set for short $L$ for the differential of the map $\Phi(t,$.$) at u$. The map $L$ is thus a continuous linear map from $C_{0}^{k+2, \alpha}$ to $C_{0}^{k, \alpha}$. We note that it can be viewed as a second order scalar differential operator $\mathcal{L}: C^{\ell+2, \alpha}(M, \mathbb{R}) \rightarrow C^{\ell, \alpha}(M, \mathbb{R})$, for every integer $\ell \leqslant k$, that is, an operator with $C^{k, \alpha}$ coefficients. Moreover, from Remark 4.1, the symbol of the operator $\mathcal{L}$ must be positive definite at every point of the manifold. So $\mathcal{L}$ is elliptic. Besides, on the one hand, since $\Phi(t, u) \equiv \Phi(t, u+$ constant $)$, the operator $\mathcal{L}$ must vanish on constant functions. On the other hand, since the integral $\int_{m} L v d V$ vanishes for every function $v \in C^{k+2, \alpha}(M, \mathbb{R})$, the 
formal $L^{2}(M, d V)$ adjoint $\mathcal{L}^{*}$ of the operator $\mathcal{L}$ must also vanish on constant functions. Hopf's maximum principle [Hop27, PW67, GT01] implies that the kernels of the operators $\mathcal{L}$ and $\mathcal{L}^{*}$ are both exactly made of the constant

functions on $M$. So the map $L: C_{0}^{k+2, \alpha} \rightarrow C_{0}^{k, \alpha}$ is not only one-to-one but also, by the Fredholm alternative combined with standard elliptic regularity, onto. The lemma now follows from the open mapping theorem

We are ready to prove that the subset $\mathcal{T}$ is relatively open in $[0,1]$. If $t_{0} \in \mathcal{T}$, letting $u_{t_{0}} \in \mathcal{A}^{k+2, \alpha}$ be a solution of (4.1) read at $t=t_{0}$, we can apply the implicit function theorem [Lan02] to the equation $\Phi(t, u)=\rho_{0}$ at the point $\left(t_{0}, u_{t_{0}}\right)$. Indeed, this point solves that equation and Lemma 4.1 tells us that the partial differential $\frac{\partial \Phi}{\partial u}\left(t_{0}, u_{t_{0}}\right)$ is an isomorphism from the tangent space to $\mathcal{A}^{k+2, \alpha}$ at $u_{t_{0}}$ to the tangent space to $P_{1}^{k, \alpha}$ at $\rho_{0}$. We infer the existence of a real $\epsilon>0$ such that $\left(t_{0}-\epsilon, t_{0}+\epsilon\right) \cap[0,1]$ is contained in $\mathcal{T}$, as desired.

\subsection{Closedness part of the method}

Our present aim is to reduce the proof that the subset $\mathcal{T}$ is closed to a uniform upper bound on the Hessian of the classical solutions $u_{t}$ of (4.1) for $t \in \mathcal{T}$. Specifically, we will establish the following statement:

Proposition 4.1. The subset $\mathcal{T}$ is closed provided there exists a constant $C_{2}>0$ such that $\nabla \operatorname{grad} u_{t} \leqslant C_{2} \mathrm{Id}$, for every $t \in \mathcal{T}$ and every solution $u_{t} \in \mathcal{A}^{k+2, \alpha}$ of the continuity equation (4.1).

Before proving the proposition, we will record straightforward, geometric or algebraic, a priori estimates bearing on admissible functions. The proof of the proposition itself is deferred till the end of the section.

Lemma 4.2. For every changing sign admissible function $u$, the following estimates hold:

$$
\max _{M}|u| \leqslant\left(D_{M}\right)^{2}, \max _{M}|\operatorname{grad} u| \leqslant D_{M},
$$

where $D_{M}$ stands for the diameter of the manifold $M$.

Proof. Let the function $u$ be admissible. By Lemma 3.5, the map $G_{u}$ is noncutting, so the length of the gradient of $u$ cannot exceed $D_{M}$. This estimate implies that the oscillation of $u$ is bounded above by $D_{M}^{2}$. So must be $\max _{M}|u|$ if $u$ changes sign

Lemma 4.3. There exists a geometric constant of the manifold $C_{M}>0$ such that $\nabla \operatorname{grad} u \geqslant-C_{M} \mathrm{Id}$, for every admissible function $u$.

Proof. If $u$ is admissible, we know from Proposition 3.1 that, for every point $m_{0} \in M$, the point $\left(m_{0}, \operatorname{grad} u\left(m_{0}\right)\right)$ must lie in $\mathcal{N}_{\text {conj }}$ and the Hessian 
endomorphism $\nabla \operatorname{grad} u\left(m_{0}\right)$ must be bounded below by $-\mathcal{J}\left(m_{0}, \operatorname{grad} u\left(m_{0}\right)\right)$. So the lemma follows from the first part of Proposition 2.3, read at $V_{0}=$ $\operatorname{grad} u\left(m_{0}\right)$, combined with the gradient bound of Lemma 4.2. Specifically, we take $C_{M}=1$ if the manifold $M$ is nonnegatively curved and, if not, we take $C_{M}=D_{M} \sqrt{\kappa_{M}} \operatorname{coth}\left(D_{M} \sqrt{\kappa_{M}}\right)$ where we have set $-\kappa_{M}<0$ for the minimum of the sectional curvature function and used Lemma 4.2

For the next statement, given a $C^{2}$ real function $u$ on $M$, it is convenient to set $c_{2}(u) \geqslant 0$ for the maximum over the manifold of the largest eigenvalue of the Hessian endomorphism $\nabla \operatorname{grad} u$. We will stick to the notation $\rho_{u}$ used above for the density of the probability measure $\mu_{u}=\left(G_{u}\right)_{\#} \mu_{0}$.

Lemma 4.4. For every admissible function $u$, the following lower bound holds:

$$
\min _{M} \operatorname{Jac}\left(\exp _{m}\right)(\operatorname{grad} u(m)) \geqslant \frac{\min _{M} \rho_{0}}{\max _{M} \rho_{u}}\left(C_{M}+c_{2}(u)\right)^{-n},
$$

where $n=\operatorname{dim}(M)$. Moreover, for every point $m \in M$, the following pinching holds in the sense of symmetric endomorphisms of the tangent space at $m$ :

$$
\frac{\min _{M} \rho_{0}}{B_{M} \max _{M} \rho_{u}}\left(C_{M}+c_{2}(u)\right)^{1-n} \operatorname{Id}_{m} \leqslant \mathcal{H} u(m) \leqslant\left(C_{M}+c_{2}(u)\right) \operatorname{Id}_{m},
$$

where $B_{M}>0$ denotes a geometric constant of the manifold.

Proof. Fixing an admissible function $u$, we note that the upper bound asserted on the Hessian endomorphism of $u$ in the lemma is trivial. From the definition of the probability density $\rho_{u}$, at every point $m$, we may write:

$$
\operatorname{Jac}\left(\exp _{m}\right)(\operatorname{grad} u(m))=\frac{\rho_{0}(m)}{\rho_{u}\left(G_{u}(m)\right)}(\operatorname{det}(\mathcal{H} u(m)))^{-1}
$$

This identity combined with the trivial upper bound on $\mathcal{H} u(m)$ yields the first lower bound of the lemma. Writing the identity the other way around, namely:

$$
\operatorname{det}(\mathcal{H} u(m))=\frac{\rho_{0}(m)}{\rho_{u}\left(G_{u}(m)\right)}\left(\operatorname{Jac}\left(\exp _{m}\right)(\operatorname{grad} u(m))\right)^{-1}
$$

and recalling the positivity of the eigenvalues of the symmetric endomorphism $\mathcal{H} u(m)$, we infer that the smallest eigenvalue of $\mathcal{H} u(m)$ is bounded below by: $\frac{\rho_{0}(m)}{\rho_{u}\left(G_{u}(m)\right)}\left(\operatorname{Jac}\left(\exp _{m}\right)(\operatorname{grad} u(m))\right)^{-1}\left(C_{M}+c_{2}(u)\right)^{1-n}$. Looking here for an upper bound on $\operatorname{Jac}\left(\exp _{m}\right)(\operatorname{grad} u(m))$, we note that, by Lemma 3.5, the tangent point $(m, \operatorname{grad} u(m))$ lies in the neighborhood $\mathcal{N}_{\text {cut }}$ of the zero section of $T M$. Bishop's comparison theorem [Gra04, p.47] thus implies that $\operatorname{Jac}\left(\exp _{m}\right)(\operatorname{grad} u(m))$ is bounded above by the constant $B_{M}$ 
equal to 1 if the manifold has nonnegative Ricci curvature and, if not, equal to $\left(\frac{\sinh \left(D_{M} \sqrt{r_{M}}\right)}{D_{M} \sqrt{r_{M}}}\right)^{n-1}$, where we have set $-(n-1) r_{M}<0$ for the smallest eigenvalue of the Ricci curvature on the manifold and used Lemma 4.2. The desired lower bound on $\mathcal{H} u(m)$ follows

Remark 4.2. The proof of Lemma 4.4 seems to indicate that the estimates 1 and 2 stated in the introduction are redundant, since one could take the constant $\delta$ in Estimate 1 equal to $\frac{\min _{M} \rho_{0}}{\max _{M} \rho_{u}} \Lambda^{-n}$. Let us explain why one cannot do so. Keeping in mind Remark 1.1, we allow $\max _{t \in[0,1]}\left|\log \rho_{t}\right|_{C^{2}}$ to enter in the control the constant $\Lambda$, whereas only $\max _{t \in[0,1]}\left|\log \rho_{t}\right|_{C^{1}}$ should enter in the control of $\delta$. Worse, the constant $\Lambda$ in Estimate 2 is allowed to depend on $\delta$. In practice, it will depend on $\delta$, because Estimate 2 will be derived from a maximum principle argument exploiting equation (4.2), in particular, the uniform boundedness of its first order term $\psi_{t}(m, \operatorname{grad} u(m))$. This boundedness will follow from Estimate 1. So one will really have to establish both estimates, one after the other, to apply successfully the continuity method.

Proof of Proposition 4.1. Recalling Remark 4.1, it is convenient to recast the continuity equation (4.1) as:

$$
F[u](m)-\psi_{t}(m, \operatorname{grad} u(m))=0, \text { with } u \in \mathcal{A}^{k+2, \alpha},
$$

where $F[u](m)=\log \operatorname{det}(\mathcal{H} u(m))$ and $\psi_{t}: \mathcal{N}_{\text {conj }} \subset T M \rightarrow \mathbb{R}$ denotes the function given by:

$$
\psi_{t}(m, V)=\log \left\{\frac{\rho_{0}(m)}{\rho_{t}\left(\exp _{m} V\right) \operatorname{Jac}\left(\exp _{m}\right)(V)}\right\} \text {, with } t \in[0,1] .
$$

Letting $\left(t_{i}\right)_{i \in \mathbb{N}}$ be a sequence of $\mathcal{T}$ and setting $t_{\infty}=\lim _{i \rightarrow \infty} t_{i} \in[0,1]$, we must prove that $t_{\infty}$ belongs to the subset $\mathcal{T}$. To each $i \in \mathbb{N}$, we associate a function $u_{i} \in \mathcal{A}^{k+2, \alpha}$ solving (4.2) at $t=t_{i}$. Under our present assumption, the three preceding lemmas hold for the function $u_{i}$, the last one with $c_{2}\left(u_{i}\right)$ replaced by the uniform constant $C_{2}$ of our statement. The sequence $\left(u_{i}\right)_{i \in \mathbb{N}}$ is thus bounded in $C^{2}(M, \mathbb{R})$. Since (4.2) is a second order equation, we would like to extract a subsequence of $\left(u_{i}\right)_{i \in \mathbb{N}}$ converging at least in $C^{2}(M, \mathbb{R})$. So we need to bound the original sequence in a stronger norm than the $C^{2}$ one. This will be achieved by means of the interior Hölder regularity theory for second derivatives of Evans-Trudinger [Eva82, Tru83, GT01]. We thus focus on verifying stepwise the conditions which this theory requires (see [GT01, section 17.4]) in the case of (4.2).

First, let us deal with the $\psi_{t}$ term. By Lemma 4.4, for every couple $(i, m) \in \mathbb{N} \times M$, the tangent point $\left(m, \operatorname{grad} u_{i}(m)\right)$ lies in a compact subset $\mathcal{K}_{1}$ (depending on the constant $C_{2}$ ) of the open neighborhood $\mathcal{N}_{\text {conj }} \subset T M$. The restriction of the function $\psi_{t}$ to the subset $\mathcal{K}_{1}$ is smooth. 
Let us now record three properties of the fully nonlinear differential operator $F$ acting on admissible functions. The differential of the operator $F$ at an admissible function $u$ is given by:

$$
d F[u](v)=\operatorname{trace}\left(\mathcal{F}_{[u]} \circ \nabla \cdot \operatorname{grad} v\right)
$$

where $\mathcal{F}_{[u]}$ stands for the endomorphism field on $M$ such that, at each point $m \in M, \mathcal{F}_{[u]}(m)$ is the symmetric endomorphism of the tangent space $T_{m} M$ inverse of the Hessian endomorphism $\mathcal{H} u(m)$. It follows from Lemma 4.4 that the symbol $\sigma(d F[u]): T^{*} M \rightarrow \mathbb{R}$ of that operator at a cotangent point $(m, \xi)$ satisfies the pinching:

$$
\begin{aligned}
& \left(C_{M}+c_{2}(u)\right)^{-1}|\xi|^{2} \leqslant \sigma(d F[u])(m, \xi) \\
& \quad \leqslant \frac{B_{M} \max _{M} \rho_{u}}{\min _{M} \rho_{0}}\left(C_{M}+c_{2}(u)\right)^{n-1}|\xi|^{2} .
\end{aligned}
$$

The operator $F$ itself factors through the function $f=\log$ det, acting on the open subset $\operatorname{End}_{\text {sym }}^{+}(T M)$ of the vector bundle $\operatorname{End}_{\text {sym }}(T M) \rightarrow M$ of symmetric endomorphisms, consisting of the ones which are positive. The restriction of the function $f$ to each fiber of the bundle is concave (for the canonical flat connection of the fiber). Back to the sequence $\left(u_{i}\right)_{i \in \mathbb{N}}$, we may infer from the pinching (4.4) read at $u=u_{i}$ a uniform pinching on the symbol $\sigma\left(d F\left[u_{i}\right]\right)(m, \xi)$, namely the one obtained by replacing the constant $c_{2}\left(u_{i}\right)$ by the constant $C_{2}$ of Proposition 4.1. As already noted, we can do similarly for the pinching of Lemma 4.4. Therefore the section $\mathcal{H} u_{i}$ of the vector bundle $\operatorname{End}_{\text {sym }}(T M)$ ranges in a compact subset $\mathcal{K}_{2}$ of $\operatorname{End}_{\text {sym }}^{+}(T M)$, depending on the constant $C_{2}$ but independent of $i \in \mathbb{N}$. The restriction of the function $f$ to the subset $\mathcal{K}_{2}$ is smooth.

We are now in position to apply the interior regularity theorem of EvansTrudinger [GT01, Theorem 17.14] to the equation (4.2) read at $u=u_{i}$. It readily yields a real $\beta \in(0,1)$, independent of $i \in \mathbb{N}$, such that the whole sequence $\left(u_{i}\right)_{i \in \mathbb{N}}$ is bounded in $C^{2, \beta}(M, \mathbb{R})$. By the Arzelà-Ascoli theorem, fixing $\gamma \in(0, \beta)$, we can extract a subsequence of $\left(u_{i}\right)_{i \in \mathbb{N}}$ which converges in $C^{2, \gamma}(M, \mathbb{R})$ toward a function $u_{\infty}$. This limit function has, like each function $u_{i}$, zero average. It must satisfy the continuity equation (4.1) read at $t=t_{\infty}$. In particular, by Lemma 3.2, it must be admissible. We infer that the differential operator $d F\left[u_{\infty}\right]$ is elliptic on the manifold. Finally, since the given probability densities $\rho_{0}$ and $\rho_{1}$ lie in $C^{k, \alpha}(M, \mathbb{R})$ and the Riemannian manifold $M$ itself is smooth, the Giraud-Hopf regularity theorem for nonlinear second order elliptic equations [Gir30, Hop31] implies that $u_{\infty}$ belongs to $C^{k+2, \alpha}(M, \mathbb{R})$, hence to $\mathcal{A}^{k+2, \alpha}$. In other words, the limit number $t_{\infty}$ belongs to the subset $\mathcal{T}$. The proof of Proposition 4.1 is thus complete 


\subsection{Conclusion: proof of the general smoothness theorem}

We wish to prove the general smoothness result stated in the introduction, namely Theorem 1.5. So far, using the continuity method, given a couple of $C^{k, \alpha}$ positive probability densities $\left(\rho_{0}, \rho_{1}\right)$, we have reduced the existence of a classical solution $u \in \mathcal{A}^{k+2, \alpha}$ of the Monge-Ampère equation (1.2), to the assumption of Proposition 4.1, that is, to the existence of a constant $C_{2}>0$ such that $\nabla \operatorname{grad} u_{t} \leqslant C_{2}$ Id, for every $t \in \mathcal{T}$ and $u_{t} \in \mathcal{A}^{k+2, \alpha}$ solving the continuity equation (4.1).

Proof of the 'if' part of Theorem 1.5. Let us show that our present existence result is equivalent to the 'if' part of Theorem 1.5, in other words, that the existence of a uniform upper bound on $\nabla \operatorname{grad} u_{t}$ is equivalent to the existence of a couple of positive constants $(\delta, \Lambda)$ such that the following bounds hold:

$$
\begin{aligned}
\operatorname{Jac}\left(\exp _{m}\right)\left(\operatorname{grad} u_{t}(m)\right) & \geqslant \delta, \\
\mathcal{J}\left(m, \operatorname{grad} u_{t}(m)\right)+\nabla \operatorname{grad} u_{t}(m) & \leqslant \Lambda \operatorname{Id}_{m},
\end{aligned}
$$

uniformly with respect to the point $(t, m) \in \mathcal{T} \times M$.

Indeed, if the preceding bound holds, we infer from Lemma 4.4, read with $u=u_{t}, \rho_{u}=\rho_{t}$ and $c_{2}(u)$ replaced by the constant $C_{2}$, that the bounds (4.5) hold as well, respectively with $\delta=\frac{\min _{M} \rho_{0}}{\max _{M} \rho_{t}}\left(C_{M}+C_{2}\right)^{-n}$ and $\Lambda=C_{M}+C_{2}$. Conversely, if the bounds (4.5) hold, we infer from (4.5a) combined with Lemma 4.2 and the estimates on $\left(\frac{\partial \exp }{\partial m}\right)_{V}^{\nabla}\left(m, \operatorname{grad} u_{t}(m)\right)$ and $\left(\frac{\partial \exp }{\partial V}\right)_{m}\left(m, \operatorname{grad} u_{t}(m)\right)$ which follow from $(2.11)$, that the norm of the Jacobi endomorphism at $\left(m, \operatorname{grad} u_{t}(m)\right)$ is bounded above by:

$$
\frac{1}{\delta} e^{\frac{n C}{2}\left(1+D_{M}^{2}\right)} n^{n / 2}
$$

where $C$ stands for the geometric constant of the manifold appearing in (2.11). In particular, a uniform lower bound on $\mathcal{J}\left(m, \operatorname{grad} u_{t}(m)\right)$ follows. The latter combined with $(4.5 \mathrm{~b})$ provides an upper bound $\nabla \operatorname{grad} u_{t} \leqslant C_{2} \mathrm{Id}$ on the Hessian endomorphism, with:

$$
C_{2}=\Lambda+\frac{1}{\delta} e^{\frac{n C}{2}\left(1+D_{M}^{2}\right)} n^{n / 2} .
$$

The 'if' part of Theorem 1.5 is thus established.

Before giving the proof of the 'only if' part of Theorem 1.5, we require the following result:

Proposition 4.2 (uniqueness). Any two $C^{2}$ solutions of the Monge-Ampère equation (1.2) on a closed manifold $M$ must differ by a constant. 
This result is a particular case of a general one presented in [Del04, Theorem A.1]. For completeness, let us provide a proof of it.

Proof. Let $w_{0}$ and $w_{1}$ be $C^{2}$ solutions of (1.2). By Lemma 3.2, each of them must be admissible. By Proposition 3.1, the $c$-Hessian of each must be positive definite everywhere on the manifold. Set $w_{\theta}=w_{0}+\theta w$ with $\theta \in[0,1]$ and $w=w_{1}-w_{0}$, and consider the nonempty closed subset $S=\left\{m \in M, w(m)=\min _{M} w\right\}$. Let us argue by connectedness in the manifold $M$ and show that the subset $S$ is open. Fixing a point $m_{0} \in S$, we note that $\operatorname{grad} w\left(m_{0}\right)$ vanishes while the symmetric endomorphism $\nabla \operatorname{grad} w\left(m_{0}\right)$ is nonnegative. We infer that the latter vanishes. Indeed, writing (1.2) at the point $m_{0}$ for the admissible functions $w_{0}$ and $w_{1}$, and noting that: $G_{w_{0}}\left(m_{0}\right)=G_{w_{1}}\left(m_{0}\right), \operatorname{Jac}\left(\exp _{m_{0}}\right)\left(\operatorname{grad} w_{0}\left(m_{0}\right)\right)=$ $\operatorname{Jac}\left(\exp _{m_{0}}\right)\left(\operatorname{grad} w_{1}\left(m_{0}\right)\right), \mathcal{J}\left(m_{0}, \operatorname{grad} w_{0}\left(m_{0}\right)\right)=\mathcal{J}\left(m_{0}, \operatorname{grad} w_{1}\left(m_{0}\right)\right)$, we infer that $\operatorname{det}\left(\mathcal{H} w_{0}\left(m_{0}\right)\right)=\operatorname{det}\left(\mathcal{H} w_{1}\left(m_{0}\right)\right)$, from which the vanishing of $\nabla \operatorname{grad} w\left(m_{0}\right)$ follows. Since the functions $w_{0}$ and $w_{1}$ are $C^{2}$, we can find a geodesic ball $B_{0}$ centered at the point $m_{0}$ on which the differential of the auxiliary operator $u \in C^{2}(M, \mathbb{R}) \rightarrow \widetilde{\Phi}(u)=\left(\rho_{1} \circ G_{u}\right) \operatorname{Jac}\left(G_{u}\right) \in C^{0}(M, \mathbb{R})$, calculated at the function $u=w_{\theta}$, stays elliptic with positive symbol, for every $\theta \in[0,1]$. So the linear operator $\widetilde{L}$, given by $\widetilde{L}=\int_{0}^{1} d \widetilde{\Phi}\left(w_{\theta}\right) d \theta$, shares the same property. Moreover, the operators $d \widetilde{\Phi}\left(w_{\theta}\right)$ and $\widetilde{L}$ are linear second order differential operators with continuous coefficients on the ball $B_{0}$ and they vanish on constant functions. The identity $\widetilde{\Phi}\left(w_{1}\right)-\widetilde{\Phi}\left(w_{0}\right) \equiv \widetilde{L}(w)$, combined with the vanishing of its left-hand side and Hopf's maximum principle [Hop27, PW67, GT01], thus implies that the function $w$ is constant on $B_{0}$, equal to $w\left(m_{0}\right)=\min _{M} w$. In other words, the ball $B_{0}$ must be contained in the subset $S$. It follows that $S$ is open hence it must coincide with the whole manifold

Proof of the 'only if' part of Theorem 1.5. By the 'only if' part of the theorem, we mean its final part, of course. So let us suppose the existence of a couple $\left(\rho_{0}, \rho_{1}\right)$ of $C^{k, \alpha}$ positive probability densities on $M$ such that either bounds (4.5) fails. By Proposition 4.2, we may consider the solution map $t \in \mathcal{T} \rightarrow u_{t} \in \mathcal{A}^{k+2, \alpha}$ defined by the continuity equation (4.1). The proof given above for the 'if' part shows that the corresponding family of functions $\left\{u_{t}\right\}_{t \in \mathcal{T}}$ must be unbounded in $C^{2}(M, \mathbb{R})$. Accordingly, for each nonzero integer $i$, we can find a real $t_{i} \in \mathcal{T}$ such that the $C^{2}$ norm of $u_{i}:=u_{t_{i}}$ is larger than $i$. Setting $\tau=\lim _{i \rightarrow \infty} t_{i} \in[0,1]$, we claim that McCann's potential function $u\left(\rho_{0} d V, \rho_{\tau} d V\right)$ is not $C^{2}$. We prove it by contradiction. If this function were $C^{2}$, the combination of Lemmas 3.1 and 3.2 would imply that it is admissible, thus satisfying the continuity equation (4.1) read at $t=\tau$. Moreover, the nonlinear elliptic regularity argument used in the last part of the proof of Proposition 4.1 would apply to the function $u\left(\rho_{0} d V, \rho_{\tau} d V\right)$ and show that it belongs to $C^{k+2, \alpha}(M, \mathbb{R})$. We 
would thus infer that $\tau$ belongs to the solution counterset $\mathcal{T}$ and, recalling Proposition 4.2, that the difference between $u\left(\rho_{0} d V, \rho_{\tau} d V\right)$ and the solution $u_{\tau} \in \mathcal{A}^{k+2, \alpha}$ is constant. Finally, by Lemma $4.1 \mathrm{read}$ at $t=\tau$, the sequence $\left(u_{i}\right)_{i \in \mathbb{N}^{*}}$ would converge toward $u_{\tau}$ in $C^{k+2, \alpha}(M, \mathbb{R})$. But this convergence would contradict the blow up of the corresponding sequence of $C^{2}$ norms

\section{Continuity method applied to flat manifolds}

Let us digress briefly from our main topic and record a straightforward application of Theorem 1.5, namely:

Theorem 5.1. If the closed Riemannian manifold $M$ is flat, given a couple $(k, \alpha) \in \mathbb{N} \times(0,1)$, with $k \geqslant 2$, the potential function $u\left(\mu_{0}, \mu_{1}\right)$ must be $C^{k+2, \alpha}$ for every couple $\left(\mu_{0}, \mu_{1}\right)$ of $C^{k, \alpha}$ positive probability measures.

As mentioned in the introduction, this theorem is known. It follows from Cordero-Erausquin's regularity result [Cor99] combined with further elliptic regularity, once applied to the manifold $M$ the Bieberbach theorem (see e.g. [KN96a, section V.4]) combined with the covering space result of [DG10, Appendix C]. Here, we present an alternative, direct proof of it.

Proof. Let us fix a couple $\left(\mu_{0}, \mu_{1}\right)$ of $C^{k, \alpha}$ positive probability measures on $M$ and apply Theorem 1.5. If $M$ is flat, the following identities hold:

$$
\operatorname{Jac}\left(\exp _{m}\right)(V) \equiv 1, \mathcal{J}(m, V) \equiv \operatorname{Id}_{m},
$$

at every tangent point $(m, V)$. In particular, the open neighborhood $\mathcal{N}_{\text {conj }}$ consists of the whole tangent bundle and the first requirement of Theorem 1.5 is trivially fulfilled. Moreover, the continuity equation reads:

$$
\log \operatorname{det}\left(\operatorname{Id}_{m}+\nabla \operatorname{grad} u_{t}(m)\right)=\psi_{t}\left(m, \operatorname{grad} u_{t}(m)\right),
$$

with $\psi_{t}(m, V)=\log \rho_{0}(m)-\log \rho_{t}\left(\exp _{m} V\right)$. Here, let us recall that the function $u_{t}$ satisfies $\int_{M} u_{t} d V=0$, hence also, by Lemma 4.2, the bounds: $\left|\operatorname{grad} u_{t}\right| \leqslant D_{M},\left|u_{t}\right| \leqslant D_{M}^{2}$. Therefore the function $u_{t}$ solves on the manifold $M$ a Monge-Ampère equation of the type studied in Corollaries 2 and 3 of [Del81]. The second requirement of Theorem 1.5 thus follows from the $a$ priori estimate carried out in [Del82, pp.428-429] to fix the proof of Lemma 6 of [Del81, p.351]. So, by Theorem 1.5, we are done

\section{Continuity method applied to A3 manifolds}

\subsection{Main statements}

Definition 6.1. A closed Riemannian manifold $(M, g)$ will be called an A3 manifold if there exists a constant $\theta>0$ such that the following inequality 
holds:

$$
-\frac{d^{2}}{d t^{2}}(g(\mathcal{J}(m, V+t W)(U), U))_{t=0} \geqslant \theta|U|^{2}|W|^{2},
$$

for every tangent point $(m, V)$ lying in $\mathcal{N}_{\text {cut }}$ and every couple of tangent vectors $(U, W) \in T_{m} M \times T_{m} M$ orthogonal to each other.

The inequality itself originates in the condition A3 of [MTW05, p.154], as will be clear from the proof of Lemma 6.1 given below. Read along the zero section of $T M$, it implies that the sectional curvature of $M$ is bounded below by $\frac{3}{2} \theta$ [DG10, p.101]. Since $\theta>0$, we infer by Rauch comparison [dC92, CE08, Jos11, Kli95] that the closure of the neighborhood $\mathcal{N}_{\text {conj }}$ in $T M$ is compact. In particular, for every real $\delta>0$ small, so is the closure of the smooth strict subdomain $\mathcal{N}_{\text {conj }}^{\delta}$ of $\mathcal{N}_{\text {conj }}$ given by:

$$
\mathcal{N}_{\text {conj }}^{\delta}=\left\{(m, V) \in \mathcal{N}_{\text {conj }}, \operatorname{Jac}\left(\exp _{m}\right)(V)>\delta\right\} .
$$

Fixing such a real $\delta$, one may thus define on the closure of $\mathcal{N}_{\text {conj }}^{\delta}$ Banach spaces of $C^{2}$ functions or sections of the pullback bundle $\varpi_{M}^{*} T M$, as explained in section 2.2. We will denote below the corresponding norms simply by $|\cdot|_{2}^{(\delta)}$. Our present aim is to derive from Theorem 1.5 the following result:

Theorem 6.1. Let $M$ be a closed A3 manifold. Given $(k, \alpha) \in \mathbb{N} \times(0,1)$, with $k \geqslant 2$, the potential function $u\left(\mu_{0}, \mu_{1}\right)$ is $C^{k+2, \alpha}$ for every couple of $C^{k, \alpha}$ positive probability measures $\left(\mu_{0}, \mu_{1}\right)$, if, for each such couple, the infimum of $\min _{m \in M} \operatorname{Jac}\left(\exp _{m}\right)\left(\operatorname{grad} u_{t}(m)\right)$, over the solution counterset $\mathcal{T}$, is positive.

Proof. To prove Theorem 6.1, we fix a couple $\left(\mu_{0}, \mu_{1}\right)=\left(\rho_{0} d V, \rho_{1} d V\right)$ of $C^{k, \alpha}$ positive probability measures on $M$ and set $\delta>0$ for the infimum, over the solution counterset $\mathcal{T}$, of $\min _{m \in M} \operatorname{Jac}\left(\exp _{m}\right)\left(\operatorname{grad} u_{t}(m)\right)$ where $u_{t}$ denotes the solution in $\mathcal{A}^{k+2, \alpha}$ of the continuity equation (4.1) corresponding to the data $\left(\rho_{0}, \rho_{1}\right)$ and the value $t \in \mathcal{T}$ of the deformation parameter. All we have to do for the proof is to combine Theorem 1.5 with the following key lemma, formulated according to Remark 1.1:

Lemma 6.1 (Ma-Trudinger-Wang estimate). There exists a constant $\Theta$ under control, depending on $\delta$, such that the trace $\mathfrak{T}_{t}(m)$ of the c-Hessian endomorphism $\mathcal{H} u_{t}(m)$ is bounded above by $\Theta$, for every point $(t, m) \in \mathcal{T} \times$ $M$.

Indeed, by Proposition 3.1, this lemma implies Estimate 2 of the introduction. Granted the lemma, the second requirement of Theorem 1.5 is thus fulfilled and the proof of Theorem 6.1 is complete. As for the proof of Lemma 6.1 itself, recalling Lemma 3.5, it may be reduced essentially to the estimate carried out in [MTW05, Section 4]. This reduction was performed in [DG10, Appendix B]; so we are done 
We would like to point out that Theorem 1.4 of [LV10] follows at once from Theorem 6.1. In other words, granted the Ma-Trudinger-Wang estimate, the PDE approach makes that result obvious. Here, the reader should not be confused by the proof outlined in [LV10, pp.436-437]. Indeed, a key fact was unobserved in [LV10] although clear from [DG10, Appendix B], namely: on every A3 manifold such that $\mathcal{N}_{\text {cut }}=\mathcal{N}_{\text {cut }} \cap \mathcal{N}_{\text {conj }}^{\delta}$ for some $\delta>0$, the two-point function $c=\frac{1}{2} d^{2}$ fulfills the assumptions made on the cost function in [MTW05]. There is thus no estimate to pursue any further.

\subsection{Maximum Principle à la Ma-Trudinger-Wang}

Instead of relying as above on the reduction to [MTW05, Section 4] operated in [DG10, Appendix B], it is instructive to give a proof of Lemma 6.1 featuring an intrinsic control on the constant $\Theta$ of the lemma. For completeness, we present such a proof in this section.

Outline of an intrinsic proof of Lemma 6.1 Fix $t \in \mathcal{T}$, pick a point $m_{0} \in M$ such that $\mathfrak{T}_{t}\left(m_{0}\right)=\max _{m \in M} \mathfrak{T}_{t}(m)$ and write successively the critical equality and the maximum inequality, namely (dropping the subscript $t$ ):

$$
\begin{aligned}
\operatorname{grad} \mathfrak{T}\left(m_{0}\right) & =0, \\
d F[u](\mathfrak{T})\left(m_{0}\right) & \leqslant 0 .
\end{aligned}
$$

To derive from (6.1) a controlled upper bound on $\mathfrak{T}\left(m_{0}\right)$, we must first cope with the third and fourth order terms appearing in (6.1b). This will be achieved by using, besides (6.1a), the continuity equation (4.2) differentiated twice. We will be left with an inequality involving terms of order at most two. To reach the desired bound on $\mathfrak{T}\left(m_{0}\right)$, we will sort these terms according to a criterion inspired from [MTW05, bottom p.162]. Let us define it, assuming with no loss of generality that $\mathfrak{T}\left(m_{0}\right) \geqslant 1$, sticking to the notations used in the proof of Proposition 4.1 (see section 4.2) and using the norms $|\cdot|_{2}^{(\delta)}$ introduced above (after Definition 6.1):

Definition 6.2. Any of the terms of order 0, 1 or 2 under study at the point $m_{0}$ will be called MTW-bounded if it can be bounded above by:

$$
C\left(1+\mathfrak{T}^{2}\left(m_{0}\right)+\mathfrak{T}\left(m_{0}\right) \operatorname{trace}\left(\mathcal{F}_{[u]}\left(m_{0}\right)\right)\right),
$$

for some positive constant $C$ controlled by the dimension $n$ and the diameter $D_{M}$ of the manifold $M$, the $C^{1}$ norm of its Riemann tensor, the norm $|\mathcal{J}|_{2}^{(\delta)}$ of the Jacobi endomorphism $\mathcal{J}$ and $\max _{t \in[0,1]}\left|\psi_{t}\right|_{2}^{(\delta)}$, where the real function $\psi_{t}$ is the one appearing in the continuity equation (4.2).

With this outline in mind, let us get to a detailed proof of Lemma 6.1. 
Some local notations. Given a chart $\left(x^{1}, \ldots, x^{n}\right)$ of the manifold $M$ and the natural chart $\left(x^{1}, \ldots, x^{n}, v^{1}, \ldots, v^{n}\right)$ associated to it on $T M$, we will set for short:

$$
\frac{\partial}{\partial x^{i}}=\partial_{i}, \frac{\partial^{2}}{\partial x^{i} \partial x^{j}}=\partial_{i j}, \frac{\partial}{\partial v^{i}}=D_{i}, \frac{\partial^{2}}{\partial v^{i} \partial v^{j}}=D_{i j}, \frac{\partial^{2}}{\partial x^{i} \partial v^{j}}=\partial_{i} D_{j}, \ldots
$$

We will take the chart $\left(x^{1}, \ldots, x^{n}\right)$ normal at the point $m_{0}$ and such that the matrix of the endomorphism $\mathcal{H} u_{t}\left(m_{0}\right)$ is diagonal, with diagonal entries $\lambda_{1} \leqslant \lambda_{2} \leqslant \ldots \leqslant \lambda_{n}$ (for simplicity, we drop the subscript $t$ until the end of the proof). Components of relevant tensors will be denoted by:

$$
\begin{gathered}
\operatorname{grad} u=\nabla^{j} u(x) \partial_{j}, \nabla \operatorname{grad} u=\nabla_{i}^{j} u(x) d x^{i} \otimes \partial_{j}, \mathcal{J}=\mathcal{J}_{i}^{j}(x, v) d x^{i} \otimes \partial_{j}, \\
\text { and } \mathcal{H} u=\mathcal{H}_{i}^{j} d x^{i} \otimes \partial_{j}, \mathcal{F}_{[u]}=\mathcal{F}_{i}^{j} d x^{i} \otimes \partial_{j}, \text { thus with } \mathcal{H}_{i}^{k} \mathcal{F}_{k}^{j}=\delta_{i}^{j} .
\end{gathered}
$$

Calculation of the conditions (6.1). Using (2.7), we find for the components of $\operatorname{grad} \mathfrak{T}$ the expression:

$$
\nabla^{j} \mathfrak{T}=\nabla^{j} \mathcal{J}_{k}^{k}-g^{j p} \Gamma_{p q}^{\ell} \nabla^{q} u D_{\ell} \mathcal{J}_{k}^{k}+D_{\ell} \mathcal{J}_{k}^{k} \nabla^{j \ell} u+\nabla^{j} \nabla_{k}^{k} u .
$$

After commuting third derivatives of $u$, the condition (6.1a) thus reads:

$$
-\nabla_{k}^{k} \nabla^{j} u=\partial_{j} \mathcal{J}_{k}^{k}+\sum_{\ell=1}^{n} D_{\ell} \mathcal{J}_{k}^{k} \partial_{j \ell} u-R_{j \ell} \partial_{\ell} u, \text { at } x=0,
$$

where the first derivatives of the local function $\mathcal{J}_{k}^{k}(x, v)$ are computed at $x=$ $0, v^{i}=\partial_{i} u(0)$, and $R_{j \ell} d x^{j} \otimes d x^{\ell}$ stands for the Ricci tensor. Differentiating the function $\mathfrak{T}$ once more yields for $(6.1 \mathrm{~b})$ the expression:

$$
\mathcal{F}_{j}^{i}\left(\nabla_{i}^{j} \nabla_{k}^{k} u+\nabla_{i}^{j} \nabla^{\ell} u D_{\ell} \mathcal{J}_{k}^{k}\right)+\text { l.o.t. } \leqslant 0, \text { at } x=0,
$$

with the lower order terms given at $x=0$ by:

$$
\begin{aligned}
\text { l.o.t. }=\mathcal{F}_{j}^{i}\left(\partial_{i j} \mathcal{J}_{k}^{k}-\frac{1}{3}\left(R_{q i j}^{\ell}+R_{j i q}^{\ell}\right) D_{\ell} \mathcal{J}_{k}^{k} \partial_{q} u\right. \\
\left.+\partial_{i} D_{\ell} \mathcal{J}_{k}^{k} \partial_{j \ell} u+\partial_{j} D_{\ell} \mathcal{J}_{k}^{k} \partial_{i \ell} u+D_{h \ell} \mathcal{J}_{k}^{k} \partial_{i h} u \partial_{j \ell} u\right),
\end{aligned}
$$

where one should sum over all indices and, again, compute the derivatives of $\mathcal{J}_{k}^{k}(x, v)$ at $x=0, v^{i}=\partial_{i} u(0)$. Here, we have used the Riemann-Cartan formula $\partial_{i} \Gamma_{j q}^{\ell}=\frac{1}{3}\left(R_{q i j}^{\ell}+R_{j i q}^{\ell}\right)$, valid at the centre of any normal chart [Car51, p.244]. Let us observe that, at $x=0$, the following identities hold:

$$
\begin{gathered}
\mathcal{F}_{j}^{i} \partial_{i \ell} u=\delta_{j}^{\ell}-\mathcal{F}_{j}^{i} \mathcal{J}_{i}^{\ell}, \mathcal{F}_{j}^{i} \partial_{j \ell} u=\delta_{\ell}^{i}-\mathcal{F}_{j}^{i} \mathcal{J}_{\ell}^{j} \\
\mathcal{F}_{j}^{i} \partial_{i h} u \partial_{j \ell} u=\partial_{h \ell} u-\mathcal{J}_{\ell}^{h}+\mathcal{F}_{j}^{i} \mathcal{J}_{i}^{h} \mathcal{J}_{\ell}^{j} .
\end{gathered}
$$

They imply that the above scalar l.o.t. is MTW-bounded. We thus focus on the other terms of the inequality (6.3), namely the third and fourth order terms. We will calculate them by differentiating twice (4.2). 
Differentiating twice the continuity equation. Applying the gradient operator to (4.2) yields in our chart $x$, for each $\ell=1, \ldots, n$, the equation:

$$
\mathcal{F}_{j}^{i} \nabla^{\ell} \mathcal{H}_{i}^{j}-\nabla^{\ell}\left(\psi_{t}(x, \operatorname{grad} u(x))\right)=0,
$$

or else, with the subscript of $\psi_{t}$ dropped:

$$
\mathcal{F}_{j}^{i} \nabla^{\ell} \nabla_{i}^{j} u=-\mathcal{F}_{j}^{i} \nabla^{\ell}\left(\mathcal{J}_{i}^{j}(x, \operatorname{grad} u(x))\right)+\nabla^{\ell}(\psi(x, \operatorname{grad} u(x))) .
$$

Commuting the third derivatives of $u$ and using (2.2)(2.7) to calculate the first derivatives of $\mathcal{J}_{i}^{j}$ and $\psi$, we get:

$$
\begin{array}{r}
\mathcal{F}_{j}^{i} \nabla_{i}^{j} \nabla^{\ell} u=\mathcal{F}_{j}^{i}\left(g^{\ell h} R_{q i h}^{j} \nabla^{q} u-\nabla^{\ell} \mathcal{J}_{i}^{j}+g^{\ell h} \Gamma_{q h}^{p} \nabla^{q} u D_{p} \mathcal{J}_{i}^{j}-D_{h} \mathcal{J}_{i}^{j} \nabla^{\ell h} u\right) \\
+\nabla^{\ell} \psi-g^{\ell \ell} \Gamma_{q i}^{j} \nabla^{q} u D_{j} \psi+D_{j} \psi \nabla^{\ell j} u .
\end{array}
$$

At $x=0$, we thus obtain the $n$ equations:

$$
\mathcal{F}_{j}^{i} \nabla_{i}^{j} \nabla^{\ell} u=\mathcal{F}_{j}^{i}\left(R_{q i \ell}^{j} \partial_{q} u-\partial_{\ell} \mathcal{J}_{i}^{j}-D_{h} \mathcal{J}_{i}^{j} \partial_{h \ell} u\right)+\partial_{\ell} \psi+D_{j} \psi \partial_{j \ell} u,
$$

where one should sum over all repeated indices and compute the first derivatives of $\mathcal{J}_{i}^{j}(x, v)$ and $\psi(x, v)$ at the point $x=0, v^{i}=\partial_{i} u(0)$. We infer from these equations combined with (6.5a) that the term $\mathcal{F}_{j}^{i} \nabla_{i}^{j} \nabla^{\ell} u D_{\ell} \mathcal{J}_{k}^{k}$ appearing in (6.3) is MTW-bounded.

We are left with treating the fourth order term of (6.3). To do so, we apply the Laplace operator to (4.2) and get:

$$
\begin{aligned}
\mathcal{F}_{j}^{i} \nabla_{k}^{k} \nabla_{i}^{j} u=\mathcal{F}_{p}^{i} \mathcal{F}_{j}^{q} \nabla_{k} \mathcal{H}_{q}^{p} \nabla^{k} \mathcal{H}_{i}^{j} & \\
& -\mathcal{F}_{j}^{i} \nabla_{k}^{k}\left(\mathcal{J}_{i}^{j}(x, \operatorname{grad} u(x))\right)-\Delta(\psi(x, \operatorname{grad} u(x))) .
\end{aligned}
$$

After commutation of the fourth derivatives of $u$, the left-hand side becomes equal to $\mathcal{F}_{j}^{i} \nabla_{i}^{j} \nabla_{k}^{k} u+$ l.o.t., with

$$
\text { l.o.t. }=\mathcal{F}_{j}^{i}\left(\left(\nabla^{k} R_{\ell k i}^{j}+\nabla_{i} R_{\ell}^{j}\right) \nabla^{\ell} u+2 R_{\ell k i}^{j} \nabla^{k \ell} u+R_{\ell i} \nabla^{\ell j} u+R_{\ell}^{j} \nabla_{i}^{\ell} u\right) \text {, }
$$

an expression which, evaluated at $x=0$, is MTW-bounded. Let us now calculate the second derivatives of $\mathcal{J}_{i}^{j}$ and $\psi$ appearing in the right-hand side of (6.6). Using (2.7) and the identity $\sum_{k=1}^{n} \partial_{k} \Gamma_{i k}^{\ell}=-\frac{1}{3} R_{i}^{\ell}$, valid at $x=0$ due to the Riemann-Cartan formula [Car51, p.244], we find that the term $-\mathcal{F}_{j}^{i} \nabla_{k}^{k}\left(\mathcal{J}_{i}^{j}(x, \operatorname{grad} u(x))\right)$, evaluated at $x=0$, is equal to:

$$
-\mathcal{F}_{j}^{i}\left(D_{\ell} \mathcal{J}_{i}^{j} \nabla_{k}^{k} \nabla^{\ell} u+D_{h \ell} \mathcal{J}_{i}^{j} \nabla_{k}^{\ell} u \nabla^{k h} u\right)+\text { further terms. }
$$

The further terms in this equation are given by the following expression:

$$
-\mathcal{F}_{j}^{i}\left(\partial_{k k} \mathcal{J}_{i}^{j}+\frac{1}{3}\left(R_{i}^{\ell} \mathcal{J}_{\ell}^{j}-R_{\ell}^{j} \mathcal{J}_{i}^{\ell}+R_{k}^{\ell} D_{\ell} \mathcal{J}_{i}^{j} \partial_{k} u\right)+\partial_{k} D_{\ell} \mathcal{J}_{i}^{j} \partial_{k \ell} u\right),
$$


where one should sum over all indices and compute the derivatives of $\mathcal{J}_{i}^{j}(x, v)$ at $x=0, v^{i}=\partial_{i} u(0)$. So, we see that the further terms into question are MTW-bounded. Besides, using (2.2), we get:

$$
-\Delta(\psi(x, \operatorname{grad} u(x)))_{x=0}=D_{\ell} \psi \nabla_{k}^{k} \nabla^{\ell} u+\text { l.o.t. }
$$

with l.o.t. $=\partial_{k k} \psi+\frac{1}{3} R_{k}^{\ell} D_{\ell} \psi \partial_{k} u+2 \partial_{k} D_{\ell} \psi \partial_{k \ell} u+D_{h \ell} \psi \partial_{k \ell} u \partial_{k h} u$, where one should sum over all indices and compute the derivatives of $\psi(x, v)$ at $x=0, v^{i}=\partial_{i} u(0)$. It is clear that the terms abbreviated here by l.o.t. are MTW-bounded. In order to cope with the third order terms $-\mathcal{F}_{j}^{i} D_{\ell} \mathcal{J}_{i}^{j} \nabla_{k}^{k} \nabla^{\ell} u$ and $D_{\ell} \psi \nabla_{k}^{k} \nabla^{\ell} u$ at $x=0$, we use the critical condition (6.2). The latter implies that these terms are MTW-bounded. Last, we note that the fourth order term $-\mathcal{F}_{j}^{i} D_{h \ell} \mathcal{J}_{i}^{j} \nabla_{k}^{\ell} u \nabla^{k h} u$ is equal to $-\mathcal{F}_{j}^{i} D_{h \ell} \mathcal{J}_{i}^{j} \mathcal{H}_{\ell}^{k} \mathcal{H}_{k}^{h}$ up to additional MTW-bounded terms. Altogether, we infer from (6.6) read at $x=0$ the inequality:

$$
\mathcal{F}_{j}^{i} \nabla_{i}^{j} \nabla_{k}^{k} u \geqslant-\sum_{j \neq k} \mathcal{F}_{j}^{j} D_{k k} \mathcal{J}_{j}^{j}\left(\mathcal{H}_{k}^{k}\right)^{2}+\text { MTW-bounded terms, }
$$

in which one should sum over all indices and compute the derivatives $D_{k k} \mathcal{J}_{j}^{j}$ at the point $x=0, v^{i}=\partial_{i} u(0)$. Here, we have used the fact that the matrices $\mathcal{F}_{j}^{i}$ and $\mathcal{H}_{\ell}^{k}$ are diagonal at $x=0$. Since these matrices are inverses of each other, we have dropped the part of the sum $-\sum_{k=1}^{n} \mathcal{F}_{k}^{k} D_{k k} \mathcal{J}_{k}^{k}\left(\mathcal{H}_{k}^{k}\right)^{2}$ obtained for $j=k$, because it is MTW-bounded.

Back to the maximum condition. Since we have assumed that the manifold $M$ is A3, Definition 6.1 implies the existence of a real $\theta>0$ such that the sum $-\sum_{j \neq k} \mathcal{F}_{j}^{j} D_{k k} \mathcal{J}_{j}^{j}\left(\mathcal{H}_{k}^{k}\right)^{2}$ is bounded below by $\theta \sum_{j \neq k} \mathcal{F}_{j}^{j}\left(\mathcal{H}_{k}^{k}\right)^{2}$. Combining this bound with (6.8) and (6.3) and recalling that $\lambda_{1} \leqslant \ldots \leqslant \lambda_{n}$ stand for the eigenvalues of the symmetric (and positive) endomorphism $\mathcal{H} u\left(m_{0}\right)$, each repeated with its multiplicity, we conclude that there exists a constant $C$, controlled as in Definition 6.2, such that the following inequality holds:

$$
\theta \sum_{j \neq k} \frac{\lambda_{k}^{2}}{\lambda_{j}} \leqslant C\left(1+\left(\sum_{k=1}^{n} \lambda_{k}\right)^{2}+\sum_{k=1}^{n} \lambda_{k} \sum_{j=1}^{n} \frac{1}{\lambda_{j}}\right) .
$$

The left-hand side is larger than $\theta \frac{\lambda_{n}^{2}}{\lambda_{1}}$ while the right-hand side is bounded above by $C\left(1+\lambda_{n}^{2}+\frac{\lambda_{n}}{\lambda_{1}}\right)$, with another suitably controlled constant $C$ (say, equal to $n^{2}$ times the preceding one). The resulting inequality reads:

$$
\lambda_{n}^{2}\left(\frac{\theta}{2 \lambda_{1}}-C\right)+\frac{\lambda_{n}}{\lambda_{1}}\left(\frac{\theta}{2} \lambda_{n}-C\right) \leqslant C .
$$


Completion of the proof of Lemma 6.1. Let us distinguish two cases: Case 1: either $\left(\frac{\theta}{2 \lambda_{1}}-C\right)$ or $\left(\frac{\theta}{2} \lambda_{n}-C\right)$ is less than 1 .

If $\left(\frac{\theta}{2} \lambda_{n}-C\right) \leqslant 1$, we get $\mathfrak{T}\left(m_{0}\right) \leqslant \frac{2}{\theta} n(C+1)$. If $\left(\frac{\theta}{2 \lambda_{1}}-C\right) \leqslant 1$, we combine this inequality with the continuity equation written as: $\prod_{i=1}^{n} \lambda_{i}=e^{\psi}$, where the function $\psi(x, v)$ is computed at $x=0, v^{i}=\partial_{i} u(0)$. It yields $\lambda_{n} \leqslant$ $e^{\psi}\left(\frac{2}{\theta}(C+1)\right)^{n-1}$, hence $\mathfrak{T}\left(m_{0}\right) \leqslant \frac{C}{\theta^{n-1}}$, with another suitable constant $C$. In either subcase, we are done.

Case 2: $\left(\frac{\theta}{2 \lambda_{1}}-C\right)>1$ and $\left(\frac{\theta}{2} \lambda_{n}-C\right)>1$.

Here, we infer from (6.9) that $\lambda_{n}^{2}+\frac{\lambda_{n}}{\lambda_{1}} \leqslant C$, which yields $\mathfrak{T}\left(m_{0}\right) \leqslant C$ with another suitably controlled constant $C$ and, again, we are done

\section{Proofs completion: first order (stay-away) esti- mates}

So far, we know by Theorem 6.1 that, on a closed A3 manifold $M$, the potential function of the optimal map $\operatorname{Mc}\left(\mu_{0}, \mu_{1}\right)$ is $C^{k+2, \alpha}$, for every couple of $C^{k, \alpha}, k \geqslant 2$, positive probability measures $\left(\mu_{0}, \mu_{1}\right)$ given on $M$, provided Estimate 1 of the Introduction can be established for each such couple $\left(\mu_{0}, \mu_{1}\right)$, when we use the continuity method with the path $\mu_{t}=t \mu_{1}+(1-t) \mu_{0}$. In this section, we will prove Theorems 1.3 and 1.4 by establishing Estimate 1 on the manifolds successively considered in these two theorems, all shown to be A3 manifolds [KM12, DR13, DG11].

\subsection{Positively curved Riemannian locally symmetric spaces}

In this subsection, we let the closed $n$-dimensional manifold $M$ be Riemannian locally symmetric, positively curved. We take it simply connected with no loss of generality [DG10, Appendix C]. Whenever a manifold is so, it must be a symmetric space (according to Cartan's theorem, see [KN96a, KN96b, CE08]) and each cut point must be a conjugate point [Cri62, Kar88]. Furthermore, all the geodesics must be simply closed of constant length [Cha70, Kar88]. We take this length equal to $2 \pi$. With this normalization, we know [Cha70, Kar88] that, for every point $(m, V) \in T M$ with $V \neq 0$, the Jacobi curvature endomorphism $Y \in T_{m} M \rightarrow R_{m}(Y, V) V \in T_{m} M$ (see the beginning of section 2.3.2) admits $\nu$ eigenvalues equal to $|V|^{2}$ and $(n-1-\nu)$ eigenvalues equal to $\frac{1}{4}|V|^{2}$. Furthermore, according to Cartan's classification (see [Kar88]), the integer $\nu$ must be equal to $n-1$ if $M$ is the sphere $\mathbb{S}^{n}$, or equal to 1 if $M$ is the complex projective space $\mathbb{C} P^{k}$, or equal to 3 if $M$ is the quaternionic projective space $\mathbb{H} P^{k}$, or equal to 7 if $M$ is the Cayley projective plane $\mathbb{O} P^{2}$, each with the appropriate sectional curvature (usual, holomorphic, quaternionic, or octonionic) equal to 1. 
Proof of Estimate 1 Sticking to the notations used in sections 4 and 6 , we fix $t \in \mathcal{T}$, that is, we fix a value of $t \in[0,1]$ for which there exists a solution $u_{t} \in \mathcal{A}^{k+2, \alpha}$ of the continuity equation (4.1). Let $m_{0} \in M$ be a point of the manifold where the function $m \mapsto \operatorname{Jac}\left(\exp _{m}\right)\left(\operatorname{grad} u_{t}(m)\right)$ assumes its global minimum. We seek a positive lower bound $\delta$ for that minimum. From (2.20), we know that the simpler function $w_{t}$ given by $w_{t}(m)=\frac{1}{2}\left|\operatorname{grad} u_{t}(m)\right|^{2}$ must assume a global maximum at the point $m_{0}$. Accordingly, recalling the ellipticity at $u=u_{t}$ of the differential operator $d F[u]$ given by $(4.2)(4.3)$, we may write the following critical equality and maximum inequality (dropping freely the subscript $t$ ):

$$
\begin{array}{r}
\operatorname{grad} w\left(m_{0}\right)=0, \\
d F[u](w)\left(m_{0}\right) \leqslant 0 .
\end{array}
$$

If $w\left(m_{0}\right)$ vanishes, we get $\operatorname{Jac}\left(\exp _{m}\right)\left(\operatorname{grad} u\left(m_{0}\right)\right)=1$, so we are done. If $w\left(m_{0}\right)>0$, let us choose a convenient chart to express the conditions (7.1). We take along the geodesic path $t \in \mathbb{R} \rightarrow \gamma_{0}(t)=\exp _{m_{0}}\left(t \operatorname{grad} u\left(m_{0}\right)\right)$ a parallel orthonormal frame field $t \mapsto\left(e_{1}(t), \ldots, e_{n}(t)\right)$ of the type described in the first paragraph of section 2.3.2. Specifically, setting $r_{0}=\left|\operatorname{grad} u\left(m_{0}\right)\right|$, we choose it such that $e_{n}(t)=\frac{1}{r_{0}} \dot{\gamma}_{0}(t)$ and the vectors $\left(e_{1}(t), \ldots, e_{\nu}(t)\right)$ (resp. $\left.\left(e_{\nu+1}(t), \ldots, e_{n-1}(t)\right)\right)$ span the eigenspace of the curvature endomorphism $R_{\gamma_{0}(t)}\left(., \dot{\gamma}_{0}(t)\right) \dot{\gamma}_{0}(t)$ associated to the eigenvalue $r_{0}^{2}$ (resp. $\frac{1}{4} r_{0}^{2}$ ). As the time parameter $t$ varies in a small enough open interval $I$ containing the segment $[0,1]$, this construction yields a Fermi chart defined in a thin enough tubular neighborhood $\mathfrak{N}$ of the image $\gamma_{0}(I)$ in the manifold $M$. The interval $I$ and the neighborhood $\mathfrak{N}$ are chosen such that, for every point $m \in \mathfrak{N}$, we can find a unique value $x^{n}=x^{n}(m)$ of the arc length parameter $x^{n}=r_{0} t$ and a unique $(n-1)$-tuple of reals $\left(x^{1}, \ldots, x^{n-1}\right)$ for which the following defining equality holds:

$$
m=\exp _{\gamma_{0}(t)}\left(x^{1} e_{1}(t)+\ldots+x^{n-1} e_{n-1}(t)\right) .
$$

In the Fermi chart $\left(x^{1}, \ldots, x^{n}\right)$ so defined, the coordinates $x^{i}$ may be viewed as cylindrical coordinates about the axis of the chart, namely the geodesic $\gamma_{0}$. Along the latter, the Riemannian metric of the manifold is osculating to the local Euclidean metric $\sum_{i=1}^{n}\left(d x^{i}\right)^{2}$. In particular, the Christoffel symbols vanish: $\Gamma_{i j}^{k}\left(0, \ldots, 0, r_{0} t\right) \equiv 0$. We keep writing $\left(x^{1}, \ldots, x^{n}, v^{1}, \ldots, v^{n}\right)$ for the natural chart of $T M$ associated to the chart $x$, and $\partial_{i}$ (resp. $D_{i}$ ) for the local vector fields $\frac{\partial}{\partial x^{i}}$ (resp. $\left.\frac{\partial}{\partial v^{i}}\right)$. We refer the reader to [Aub98, Gra04] or [DG10, section 2] for an account on Fermi charts. In the present Fermi chart, the critical condition (7.1a) reads:

$$
\partial_{\text {in }} u(0)=0
$$


for every integer $i=1, \ldots, n$. Besides, we get:

$$
\nabla_{i}^{j} w(0)=r_{0} \partial_{n i j} u(0)+r_{0}^{2} R_{i n j n}(0)+\sum_{\gamma=1}^{n-1} \partial_{i \gamma} u(0) \partial_{j \gamma} u(0),
$$

after commuting third covariant derivatives of the function $u$, where $R_{i j k \ell}(x)$ stand for the chart components of the covariant Riemann tensor. Recalling (4.3), the local expression of $d F[u](w)\left(m_{0}\right)$ is obtained by contracting the right-hand side of the preceding equation with the symmetric matrix $\mathcal{F}_{j}^{i}(0)$. Here comes the reason why we have chosen the elliptic operator $d F[u]$ to express the maximum condition in $(7.1 \mathrm{~b})$. Indeed, we can take advantage of the continuity equation (4.2) itself, differentiated with respect to the arc length coordinate $x^{n}$ at $x=0$, to calculate the third order term $r_{0} \mathcal{F}_{j}^{i}(0) \partial_{n i j} u(0)$ and express it by lower order terms. Specifically, we infer for $\mathcal{F}_{j}^{i}(0) \partial_{n i j} u(0)$ the expression:

$\mathcal{F}_{j}^{i}(0) \partial_{n i j} u(0)=-\left.\mathcal{F}_{j}^{i}(0) \partial_{n}\left(\mathcal{J}_{i}^{j}(x, \operatorname{grad} u(x))\right)\right|_{x=0}+\left.\partial_{n}\left(\psi_{t}(x, \operatorname{grad} u(x))\right)\right|_{x=0}$.

Combining (2.7) with (7.2), we find that each term $\partial_{n}\left(\mathcal{J}_{i}^{j}(x, \operatorname{grad} u(x))\right)$ reduces at $x=0$ to $\left(\partial_{n} \mathcal{J}_{i}^{j}\right)(0, \operatorname{grad} u(0))$. Moreover, the local symmetry of the manifold $M$ forces the latter derivative to vanish. Indeed, the components of the Jacobi endomorphism $\mathcal{J}\left(\gamma_{0}(t), \dot{\gamma}_{0}(t)\right)$ in the parallel frame $\left(e_{1}(t), \ldots, e_{n}(t)\right)$ must be constant. Specifically, by the special choice of the frame, one can readily verify (as in [DR13]) that the vector $e_{i}(t)$ is an eigenvector of $\mathcal{J}\left(\gamma_{0}(t), \dot{\gamma}_{0}(t)\right)$ associated to the eigenvalue $\lambda_{i}$ with: $\lambda_{i}=r_{0} \cot r_{0}$ if $i \leqslant \nu, \lambda_{i}=\frac{r_{0}}{2} \cot \frac{r_{0}}{2}$ if $i \in\{\nu+1, \ldots, n-1\}$ and $\lambda_{n}=1$ as always (by Proposition 2.1). So, the section $t \mapsto \mathcal{J}\left(\gamma_{0}(t), \dot{\gamma}_{0}(t)\right)$ of the pullback bundle $\gamma_{0}^{*} \operatorname{End}(T M) \rightarrow \mathbb{R}$ is parallel. The equation $\frac{\nabla}{d t} \mathcal{J}\left(\gamma_{0}(t), \dot{\gamma}_{0}(t)\right)=0$, expressed using (2.7), read at $t=0$ in the Fermi chart yields $\left(\partial_{n} \mathcal{J}_{i}^{j}\right)(0, \operatorname{grad} u(0)) \equiv 0$, as claimed. Besides, the term $\partial_{n}\left(\psi_{t}(x, \operatorname{grad} u(x))\right)$ calculated at $x=0$ is merely equal to:

$$
\left.\partial_{n}\left(\psi_{t}(x, \operatorname{grad} u(x))\right)\right|_{x=0}=\frac{\partial_{n} \rho_{0}}{\rho_{0}}(0)-\frac{\partial_{n} \rho_{t}}{\rho_{t}}\left(0, \ldots, 0, r_{0}\right),
$$

because $x=0$ is a critical point of the chart expression of the function $m \mapsto \operatorname{Jac}\left(\exp _{m}\right)(\operatorname{grad} u(m))$. We conclude that the maximum condition (7.1b) reads:

$$
\begin{aligned}
0 \geqslant \mathcal{F}_{j}^{i}(0)\left(r_{0}^{2} R_{\text {injn }}(0)+\sum_{\gamma=1}^{n-1} \partial_{i \gamma} u(0) \partial_{j \gamma} u(0)\right) & \\
& \quad+r_{0}\left(\frac{\partial_{n} \rho_{0}}{\rho_{0}}(0)-\frac{\partial_{n} \rho_{t}}{\rho_{t}}\left(0, \ldots, 0, r_{0}\right)\right) .
\end{aligned}
$$


By (6.5b) and the special choice of our chart, we get:

$$
\sum_{\gamma=1}^{n-1} \mathcal{F}_{j}^{i}(0) \partial_{i \gamma} u(0) \partial_{j \gamma} u(0)=\sum_{\gamma=1}^{n-1} \partial_{\gamma \gamma} u(0)-\mathcal{J}_{\gamma}^{\gamma}+\mathcal{F}_{\gamma}^{\gamma}(0)\left(\mathcal{J}_{\gamma}^{\gamma}\right)^{2}
$$

where the components $\mathcal{J}_{\gamma}^{\gamma}$ should be evaluated at $(0, \operatorname{grad} u(0))$. But $\partial_{\gamma \gamma} u(0)$ is equal to $\mathcal{H}_{\gamma}^{\gamma}(0)-\mathcal{J}_{\gamma}^{\gamma}(0, \operatorname{grad} u(0))$. By Proposition 3.1, it is thus bounded below by $-\mathcal{J}_{\gamma}^{\gamma}(0, \operatorname{grad} u(0))$. As for the term $\sum_{\gamma=1}^{n-1} \mathcal{F}_{\gamma}^{\gamma}(0)\left(\mathcal{J}_{\gamma}^{\gamma}\right)^{2}$, we know by (4.4) that it is nonnegative. So is the term $r_{0}^{2} \mathcal{F}_{j}^{i}(0) R_{\text {injn }}(0)$, because the manifold is positively curved. Altogether, we infer from (7.3) the inequality:

$$
0 \geqslant-2 \sum_{\gamma=1}^{n-1} \mathcal{J}_{\gamma}^{\gamma}(0, \operatorname{grad} u(0))+r_{0}\left(\frac{\partial_{n} \rho_{0}}{\rho_{0}}(0)-\frac{\partial_{n} \rho_{t}}{\rho_{t}}\left(0, \ldots, 0, r_{0}\right)\right) \text {. }
$$

Recalling Lemma 4.2, it yields the lower bound:

$$
\sum_{\gamma=1}^{n-1} \mathcal{J}_{\gamma}^{\gamma}(0, \operatorname{grad} u(0)) \geqslant-\frac{1}{2} D_{M}\left(\max _{M}\left|d \log \rho_{0}\right|+\max _{M}\left|d \log \rho_{t}\right|\right) .
$$

Here, the diameter $D_{M}$ of the manifold is equal to $\pi$ and the expression of the left-hand side is known [DR13], given by:

$$
\sum_{\gamma=1}^{n-1} \mathcal{J}_{\gamma}^{\gamma}(0, \operatorname{grad} u(0))=\nu r_{0} \cot r_{0}+(n-\nu-1) \frac{r_{0}}{2} \cot \frac{r_{0}}{2} .
$$

We thus obtain the uniform lower bound:

$$
\nu r_{0} \cot r_{0}+(n-\nu-1) \frac{r_{0}}{2} \cot \frac{r_{0}}{2} \geqslant-\pi \max _{t \in[0,1]} \max _{M}\left|d \log \rho_{t}\right| .
$$

It yields the existence of a small real $\alpha>0$, independent of the point $m_{0} \in$ $M$ and of the value $t$ of the deformation parameter in $\mathcal{T} \subset[0,1]$, such that: $r_{0}=\left|\operatorname{grad} u_{t}\left(m_{0}\right)\right| \leqslant \pi-\alpha$. Recalling (2.20), we infer Estimate 1 with $\delta=\frac{1}{(\pi-\alpha)^{n-1}}(\sin \alpha)^{\nu}\left(2 \cos \frac{\alpha}{2}\right)^{n-1-\nu}$. The proof of Theorem 1.3 is complete.

\subsection{Nearly spherical surfaces}

In this section, the manifold $M$ is the 2 -sphere endowed with a Riemannian metric $g$ of positive Gauss curvature $K$ normalized by $\min _{M} K=1$. We set henceforth $\varepsilon_{K}=|K-1|_{C^{2}(M)}$ and assume that $\varepsilon_{K}$ is small. By Myers theorem [CE08], the diameter of $M$ satisfies: $D \leqslant \pi$. In this setting, before attacking Estimate 1, we require estimates on further geometric quantities. 


\subsubsection{Two-dimensional perturbation tools}

For each point $(m, V) \in T M$ and $i=0,1$, let $t \in[0,+\infty) \rightarrow f_{i}(m, V, t) \in \mathbb{R}$ denote the solution of the Cauchy problem:

$$
\ddot{f}_{i}+|V|^{2} K\left(\exp _{m}(t V)\right) f_{i}=0, f_{i}(0)=\delta_{i 0}, \dot{f}_{i}(0)=\delta_{i 1},
$$

where the dot stands for $\frac{\partial}{\partial t}$. The ODE which appears in (7.4) is the scalar Jacobi equation. The functions $f_{0}, f_{1}$ so defined on $T M \times \mathbb{R}$ yield the solution of the Cauchy problem for the, possibly inhomogeneous, scalar Jacobi equation. Let us describe how. Fix a point $(m, V) \in T M$ with $V \neq 0$, set $\gamma(t)=\exp _{m}(t V)$ and let $t \mapsto\left(e_{1}(t), e_{2}(t)\right)$ be the direct orthonormal parallel frame field along the path $t \mapsto \gamma(t)$, such that $\dot{\gamma}(t)=|V| e_{2}(t)$. If $Y(t)=Y^{1}(t) e_{1}(t)+Y^{2}(t) e_{2}(t)$ is a Jacobi field along the geodesic $\gamma$, its first component satisfies the above scalar Jacobi equation while the component $Y^{2}$ must be an affine function of the time parameter $t$. We infer from (2.8)

that the matrices of the linear maps $\left(\frac{\partial \exp }{\partial m}\right)_{V}^{\nabla}(m, t V)$, and $t\left(\frac{\partial \exp }{\partial V}\right)_{m}(m, t V)$, in the source frame $\left(e_{1}(0), e_{2}(0)\right)$ and the target frame $\left(e_{1}(t), e_{2}(t)\right)$, are respectively equal to:

$$
\left(\begin{array}{cc}
f_{0}(m, V, t) & 0 \\
0 & 1
\end{array}\right) \text { and }\left(\begin{array}{cc}
f_{1}(m, V, t) & 0 \\
0 & t
\end{array}\right) .
$$

For each prescribed couple of reals $(a, b)$ and continuous function $t \mapsto h(t)$, it follows now from (2.10), read with $U(t)=h(t) e_{1}(t), \xi=a e_{1}(0), \nu=b e_{1}(0)$, that the solution of the Cauchy problem:

$$
\ddot{f}+|V|^{2} K\left(\exp _{m}(t V)\right) f=h, f(0)=a, \dot{f}(0)=b,
$$

must be given by:

$$
f(t)=a f_{0}(m, V, t)+b f_{1}(m, V, t)+\int_{0}^{t} f_{1}(\gamma(\tau), \dot{\gamma}(\tau), t-\tau) h(\tau) d \tau .
$$

Another consequence of (7.5) is the identity:

$$
\operatorname{Jac}\left(\exp _{m}\right)(V)=f_{1}(m, V, 1),
$$

from which we anticipate the need, for Estimate 1, to control the function $f_{1}(m, V, 1)$ together with its first and second derivatives. Before focussing on that control problem, we observe that the neighborhhood $\mathcal{N}_{\text {conj }}$ admits in dimension 2 the following characterization:

$$
\mathcal{N}_{\text {conj }}=\left\{(m, V) \in T M, \forall t \in[0,1], f_{1}(m, V, t)>0\right\} .
$$

Finally, if the point $(m, V)$ lies in $\mathcal{N}_{\text {conj }}$, we get at once from (7.5) the expression of the Jacobi endomorphism at $(m, V)$ in the tangent basis $\left(e_{1}(0), e_{2}(0)\right)$, namely:

$$
\mathcal{J}(m, V)=\left(\begin{array}{cc}
\frac{f_{0}(m, V, 1)}{f_{1}(m, V, 1)} & 0 \\
0 & 1
\end{array}\right) .
$$


Let us set $\bar{f}_{0}$ and $\bar{f}_{1}$ for the functions defined analogously to $f_{0}, f_{1}$ when the function $K$ is replaced by 1 in (7.4), that is, we set: $\bar{f}_{0}=\cos (|V| t)$ and $\bar{f}_{1}(m, V, t)=\frac{\sin (|V| t)}{|V|}$. By Sturm comparison [dC92, pp.238-239], the curvature lower bound $K \geqslant 1$ implies that the upper bound:

$$
f_{1}(m, V, t) \leqslant \bar{f}_{1}(m, V, t),
$$

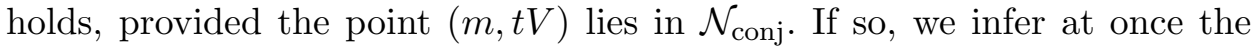
further bound:

$$
f_{1}(m, V, t) \leqslant t .
$$

Plugging this bound under the integral sign in (7.6), we get the estimate:

$$
\left\|f-a f_{0}(m, V, .)-b f_{1}(m, V, .)\right\| \leqslant \frac{1}{2}\|h\|,
$$

bearing on the solution $f$ of the inhomogeneous Cauchy problem, where the norm $\|$.$\| is defined by: \|h\|=\max _{t \in[0,1]}|h(t)|$. Let us apply this estimate to the function $f(t)=f_{1}(m, V, t)-\bar{f}_{1}(m, V, t)$, thus with $a=b=0$ and $h(t)=$ $|V|^{2}\left(1-K\left(\exp _{m}(t V)\right)\right) \bar{f}_{1}(m, V, t)$. With this choice of $f$, the combination of (7.10) with the curvature pinching yields the lower bound:

$$
f_{1}(m, V, t) \geqslant \bar{f}_{1}(m, V, t)-\pi^{2} \varepsilon_{K},
$$

valid for every $(m, V) \in \mathcal{N}_{\text {conj }}$ and $t \in[0,1]$. If $|V| \geqslant \frac{3 \pi}{4}$, this inequality read at $t=1$ implies:

$$
\pi-|V| \leqslant \arcsin \left(\pi^{3} \varepsilon_{K}+\pi f_{1}(m, V, 1)\right)
$$

hence the existence of a universal constant $C>0$ (equal to $\pi^{3} \sqrt{2}$ ) such that:

$$
\pi-|V| \leqslant C\left(\varepsilon_{K}+f_{1}(m, V, 1)\right) .
$$

So, the smaller $\varepsilon_{K}$ and $f_{1}(m, V, 1)$ are, the closer the length of the vector $V$ must get to $\pi$.

If the map $t \in[0,1] \rightarrow \gamma(t)=\exp _{m}(t V) \in M$ is one to one, we may associate to the orthonormal moving frame $\left(e_{1}(t), e_{2}(t)\right)$ defined above a Fermi chart $x=\left(x^{1}, x^{2}\right)$ along the corresponding segment. In that chart, the Riemannian metric $g$ reads:

$$
g=d x^{1} \otimes d x^{1}+\mathfrak{G}\left(x^{1}, x^{2}\right) d x^{2} \otimes d x^{2},
$$

with $\mathfrak{G}\left(0, x^{2}\right)=1, \partial_{1} \mathfrak{G}\left(0, x^{2}\right)=0$. In the domain of the chart, the Christoffel symbols vanish except the following ones:

$$
\Gamma_{22}^{1}=-\frac{1}{2} \partial_{1} \mathfrak{G}, \Gamma_{12}^{2}=\frac{\partial_{1} \mathfrak{G}}{2 \mathfrak{G}}, \Gamma_{22}^{2}=\frac{\partial_{2} \mathfrak{G}}{2 \mathfrak{G}} .
$$


Moreover, the Gauss curvature is given by: $K=-\frac{\partial_{11} \sqrt{\mathfrak{G}}}{\sqrt{\mathfrak{G}}}$. We infer that, along the $x^{2}$-axis, the first and second partial derivatives with respect to $x^{1}$ of the Christoffel symbols can be expressed in terms of $K$ as follows:

$$
\begin{aligned}
\partial_{1} \Gamma_{22}^{1}=-\partial_{1} \Gamma_{12}^{2}=K, & \partial_{1} \Gamma_{22}^{2}=0, \text { and } \\
& \partial_{11} \Gamma_{22}^{1}=-\partial_{11} \Gamma_{12}^{2}=\partial_{1} K, \partial_{11} \Gamma_{22}^{2}=-\partial_{2} K .
\end{aligned}
$$

We set $(x, v)=\left(x^{1}, x^{2}, v^{1}, v^{2}\right)$ for the natural chart of $T M$ associated to the chart $x$ and, $X=X(x, v, t)=\left(X^{1}(x, v, t), X^{2}(x, v, t)\right)=x\left(\exp _{m}(t V)\right)$, with $x=x(m), v=d x(m)(V)$, for the local expression of the geodesic motion. Besides, we still set $D_{i}=\frac{\partial}{\partial v^{i}}$ (while $\partial_{i}$ stands as above for $\frac{\partial}{\partial x^{i}}$ ). From (7.5), we get at once:

$$
\left(\begin{array}{cc}
\partial_{1} X^{1} & \partial_{2} X^{1} \\
\partial_{1} X^{2} & \partial_{2} X^{2}
\end{array}\right)=\left(\begin{array}{cc}
f_{0} & 0 \\
0 & 1
\end{array}\right),\left(\begin{array}{cc}
D_{1} X^{1} & D_{2} X^{1} \\
D_{1} X^{2} & D_{2} X^{2}
\end{array}\right)=\left(\begin{array}{cc}
f_{1} & 0 \\
0 & t
\end{array}\right)
$$

where each entry of the matrices should be calculated at the point $(x, v, t)=$ $(0,0,0,|V|, t)$, and the functions $f_{i}(x, v, t), i=0,1$, stand abusively for the local expressions of the global functions $f_{i}$ 's precedingly defined.

When the point $(m, V)$ lies in $\mathcal{N}_{\text {conj }}$, the identity (7.7) prompts us to evaluate the first and second derivatives with respect to the Fermi variables $x^{j}$ and $v^{k}$, at the point $(x, v, t)=(0,0,0,|V|, t)$, of the function $f_{1}$. If we first do it for the function $\bar{f}_{1}$ instead, we find that these derivatives, evaluated at $(0,0,0,|V|, t)$, all vanish except the following ones:

$$
D_{2} \bar{f}_{1}, \partial_{11} \bar{f}_{1}, D_{11} \bar{f}_{1}, D_{22} \bar{f}_{1} \text {. }
$$

As the length of the vector $V$ grows to $\pi$, the limits of the latter at $t=1$ are respectively equal to: $-\frac{1}{\pi}, 1,-\frac{1}{\pi^{2}}, \frac{2}{\pi^{2}}$. Let us now describe correspondingly the behavior of the function $f_{1}$.

Proposition 7.1. Let $(m, V)$ be a point of $\mathcal{N}_{\text {conj }}$ such that the geodesic path $t \in[0,1] \rightarrow \exp _{m}(t V) \in M$ is one to one. Let $x$ be the Fermi chart associated to it as done above. There exists a universal constant $C>0$ such that, for each $t \in[0,1]$, all the first and second partial derivatives of the function $f_{1}$ with respect to the Fermi variables $\left(x^{1}, x^{2}, v^{1}, v^{2}\right)$, evaluated at the point $(x, v, t)=(0,0,0,|V|, t)$, have their absolute value bounded above by $C \varepsilon_{K}$, but the following ones:

$$
D_{2} f_{1}, \partial_{11} f_{1}, D_{11} f_{1}, D_{22} f_{1} \text {. }
$$

Regarding the latter, evaluated at $(0,0,0,|V|, 1)$ with $|V| \geqslant \frac{3 \pi}{4}$, the following 
estimates hold:

$$
\begin{aligned}
\left|D_{2} f_{1}+\frac{1}{\pi}\right| & \leqslant C\left(\varepsilon_{K}+f_{1}(0,0,0,|V|, 1)\right), \\
\left|\partial_{11} f_{1}-1\right| & \leqslant C\left(\varepsilon_{K}+f_{1}(0,0,0,|V|, 1)\right), \\
\left|D_{11} f_{1}+\frac{1}{\pi^{2}}\right| & \leqslant C\left(\varepsilon_{K}+f_{1}(0,0,0,|V|, 1)\right), \\
\left|D_{22} f_{1}-\frac{2}{\pi^{2}}\right| & \leqslant C\left(\varepsilon_{K}+f_{1}(0,0,0,|V|, 1)\right) .
\end{aligned}
$$

This may be the right place to mention that the curvature of the piece of curve in $T_{m} M$ given by the local equation $f_{1}(0,0, ., ., 1)=f_{1}(0,0,0,|V|, 1)$ is equal, at the tangent vector $v=(0,|V|)$, to $\mathrm{k}=-\frac{D_{11} f_{1}}{D_{2} f_{1}}(0,0,0,|V|, 1)$. We thus see from $(7.15 \mathrm{a})(7.15 \mathrm{c})$ that, the smaller $\varepsilon_{K}$ and $f_{1}(0,0,0,|V|, 1)$ are, the closer to $-\frac{1}{\pi}$ is the curvature of that piece of curve, that is, the more that piece of curve looks in the tangent plane $T_{m} M$ like a piece of the circle of radius $\pi$ centered at the origin. For details on this matter, see [DG11, Corollary 2]. This geometrical fact will appear essential for Estimate 1.

Proof for Proposition 7.1. The proof of the proposition is a lengthy step by step procedure similar to the one used for proving the first part of Lemma 1 in [DG11]. For completeness, we indicate it to the reader as a sequence of ten guided exercises.

Exercise 1 Find the expression $X(x, v, t)$ of the geodesic motion, when $x=0$ and $v=(0,|V|)$. Using the latter and setting $\mathcal{K}(x, v, t)=K(X(x, v, t))$, calculate $\dot{\mathcal{K}}$ and $\ddot{\mathcal{K}}$ (the dot standing for $\frac{\partial}{\partial t}$ ) when $x=0$ and $v=(0,|V|)$. Using (7.14), calculate the first partial derivatives of $\mathcal{K}$ with respect to the $x$ and $v$ variables at $x=0, v=(0,|V|)$.

Exercise 2 Viewing the ODE satisfied by the $\bar{f}_{i}$ 's as a perturbation of the actual scalar Jacobi equation and using (7.6)(7.10), show the existence of a universal constant $C$ (in the sequel of the proof, we will freely denote so any constant of the sort) such that, for $i=0,1$, the norms $\left\|f_{i}-\bar{f}_{i}\right\|$ and $\left\|\dot{f}_{i}-\dot{\bar{f}}_{i}\right\|$ are bounded above by $C \varepsilon_{K}$ (this was already done above for $\left\|f_{1}-\bar{f}_{1}\right\|$ to derive (7.11)).

Exercise 3 The first partial derivatives of the function $f_{1}(x, v, t)$ with respect to the $x$ and $v$ variables, evaluated at $x=0, v=(0,|V|)$, satisfy inhomogeneous scalar Jacobi equations and have zero Cauchy data at $t=0$. Using (7.6)(7.10), show that, if we ignore the case of $D_{2} f_{1}$, the $\|\cdot\|$ norm of each of the remaining partial derivatives of $f_{1}$ is bounded above by $C \varepsilon_{K}$. 
Exercise 4 Show that $\left\|D_{2} f_{1}-D_{2} \bar{f}_{1}\right\|$, evaluated at $x=0, v=(0,|V|)$, is bounded above by $C \varepsilon_{K}$ and, using (7.12), infer the estimate (7.15a).

Exercise 5 Using (7.14), show that, if $i=j=1$, the second partial derivatives: $\partial_{2 j} X^{i}, \partial_{2} D_{j} X^{i}, D_{2} \partial_{j} X^{i}, D_{2 j} X^{i}$, evaluated at $(0,0,0,|V|, t)$, are respectively equal to: $\partial_{2} f_{0}, \partial_{2} f_{1}, D_{2} f_{0}, D_{2} f_{1}$, evaluated at the same point while, if $(i, j) \neq(1,1)$, they vanish. For each of the nonzero ones, using (7.13), write down the, possibly inhomogeneous, scalar Jacobi equation that it solves. Combining Exercises 1 and 2 with (7.6)(7.10), prove that each of the preceding nonzero second partial derivative of $X^{i}$ has a $\|$.$\| norm at the$ point $x=0, v=(0,|V|)$ bounded above by $C\left(1+\varepsilon_{K}\right)$.

Exercise 6 Regarding the six remaining second partial derivatives of $X^{i}$, evaluated at $(0,0,0,|V|, t)$, the reader will admit that they satisfy the following inhomogeneous scalar Jacobi equations (obtained by differentiating twice the geodesic equation, see equation (49) of [DG10] read with $n=2$ ):

$$
\begin{aligned}
& \frac{\partial^{2}}{\partial t^{2}}\left(\partial_{11} X^{1}\right)+|V|^{2} \mathcal{K} \partial_{11} X^{1}=-|V|^{2}\left(\partial_{1} K \circ X\right) f_{0}^{2}, \\
& \frac{\partial^{2}}{\partial t^{2}}\left(\partial_{11} X^{2}\right)=|V|^{2}\left(\partial_{2} K \circ X\right) f_{0}^{2}+4|V| \mathcal{K} f_{0} \dot{f}_{0}, \\
& \frac{\partial^{2}}{\partial t^{2}}\left(\partial_{1} D_{1} X^{1}\right)+|V|^{2} \mathcal{K} \partial_{1} D_{1} X^{1}=-|V|\left(\partial_{1} K \circ X\right) f_{0} f_{1}, \\
& \frac{\partial^{2}}{\partial t^{2}}\left(\partial_{1} D_{1} X^{2}\right)=|V|^{2}\left(\partial_{2} K \circ X\right) f_{0} f_{1}+2|V| \mathcal{K}\left(f_{0} \dot{f}_{1}+\dot{f}_{0} f_{1}\right), \\
& \frac{\partial^{2}}{\partial t^{2}}\left(D_{11} X^{1}\right)+|V|^{2} \mathcal{K} D_{11} X^{1}=-|V|^{2}\left(\partial_{1} K \circ X\right) f_{1}^{2}, \\
& \frac{\partial^{2}}{\partial t^{2}}\left(D_{11} X^{2}\right)=|V|^{2}\left(\partial_{2} K \circ X\right) f_{1}^{2}+4|V| \mathcal{K} f_{1} \dot{f}_{1} .
\end{aligned}
$$

In the same way we did at the end of the preceding exercise, show that each of these six second partial derivative of $X$ has a $\|$.$\| norm at the point$ $x=0, v=(0,|V|)$ bounded above by $C\left(1+\varepsilon_{K}\right)$.

Exercise 7 As a sequel to Exercise 1, calculate the second partial derivatives of the function $\mathcal{K}$ with respect to the $x$ and $v$ variables at $(0,0,0,|V|, t)$. Infer from Exercises 5 and 6 that each of these second partial derivative of $\mathcal{K}$ has a $\|$.$\| norm at the point x=0, v=(0,|V|)$ bounded above by $C \varepsilon_{K}$.

Exercise 8 The second partial derivatives of the function $f_{1}(x, v, t)$ with respect to the variables $x, v$, at $x=0, v=(0,|V|)$, satisfy inhomogeneous scalar Jacobi equations and have zero Cauchy data at $t=0$. Write down these Jacobi equations. Combining Exercise 7 with (7.6)(7.10) and ignoring $\partial_{11} f_{1}, D_{11} f_{1}, D_{22} f_{1}$, show that the $\|$.$\| norm of each of the remaining second$ partial derivatives is bounded above above by $C \varepsilon_{K}$. 
Exercise 9 Write down the inhomogeneous ODE's of Jacobi type satisfied by $\partial_{11} \bar{f}_{1}, D_{11} \bar{f}_{1}, D_{22} \bar{f}_{1}$, at $x=0, v=(0,|V|)$. Calculate the expressions of these second derivatives.

Exercise 10 Treating each of the ODE's found in the preceding exercise as a perturbation of the scalar Jacobi equation satisfied by the corresponding second partial derivative of $f_{1}$, prove with the help of $(7.6)(7.10)$ that the $\|$. $\|$ norms of $\partial_{11}\left(f_{1}-\bar{f}_{1}\right), D_{11}\left(f_{1}-\bar{f}_{1}\right), D_{22}\left(f_{1}-\bar{f}_{1}\right)$, at $x=0, v=(0,|V|)$, are bounded above by $C \varepsilon_{K}$. Combining (7.12) with the last part of Exercise 9 , infer the estimates $(7.15 \mathrm{~b})(7.15 \mathrm{c})(7.15 \mathrm{~d})$.

We will also require results of the same kind bearing on the function $f_{0}$. We record them here without proof:

Proposition 7.2. Under the assumption made on the point $(m, V)$ at the beginning of Proposition 7.1, there exists a universal constant $C>0$ such that, for each $t \in[0,1]$, the first partial derivatives of the function $f_{0}$ with respect to the Fermi variables $x^{1}, x^{2}, v^{1}$ (excepting $v^{2}$ ), evaluated at the point $(x, v, t)=(0,0,0,|V|, t)$, have their absolute value bounded above by $C \varepsilon_{K}$. Moreover, if $|V| \geqslant \frac{3 \pi}{4}$, the following estimates hold:

$$
\begin{gathered}
\left|f_{0}(0,0,0,|V|, 1)+1\right| \leqslant C\left(\varepsilon_{K}+f_{1}(0,0,0,|V|, 1)\right), \\
\left|D_{2} f_{0}(0,0,0,|V|, 1)\right| \leqslant C\left(\varepsilon_{K}+f_{1}(0,0,0,|V|, 1)\right) .
\end{gathered}
$$

\subsubsection{Analytic set up at the minimum point}

The start of Estimate 1 is standard. We fix $t \in \mathcal{T}$ and let $m_{0} \in M$ be a point where the function $m \mapsto \operatorname{Jac}\left(\exp _{m}\right)\left(\operatorname{grad} u_{t}(m)\right)$ assumes its minimum. We seek a positive lower bound under control on that minimum. We will derive it by writing at the point $m_{0}$ the critical equality and the minimum inequality satisfied by the preceding function and by combining them with the continuity equation (4.2) satisfied by the potential function $u_{t}$. In this process, we will have the freedom to shrink the curvature pinching parameter $\varepsilon_{K}$ as long as its size stays independent of the given density functions $\rho_{0}, \rho_{t}$.

By (7.7), we know that the test function which we are minimizing on the manifold is nothing but the function $m \mapsto f_{1}\left(m, \operatorname{grad} u_{t}(m), 1\right)$. Henceforth, we drop freely the subscript $t$ and set for short:

$$
\phi_{1}(m)=f_{1}(m, \operatorname{grad} u(m), 1), \delta_{0}=\phi_{1}\left(m_{0}\right), V_{0}=\operatorname{grad} u\left(m_{0}\right), r_{0}=\left|V_{0}\right| .
$$

Here, we will be able to shrink the parameter $\delta_{0}$ as much as needed, provided it remains under control (in the sense of Remark 1.1, still). We assume that the length $r_{0}$ of the vector $V_{0}$ is greater than $\frac{3 \pi}{4}$. We may do so because (7.11), read at $t=1$ with $|V| \leqslant \frac{3 \pi}{4}$, yields the lower bound $f_{1}(m, V, 1) \geqslant$ 
$\frac{2 \sqrt{2}}{3 \pi}-\pi^{2} \varepsilon_{K}$ which can be made positive by taking $\varepsilon_{K}$ small enough. Besides, it is clear from Lemma 3.5 that the geodesic path $t \in[0,1] \rightarrow \gamma_{0}(t)=$ $\exp _{m_{0}}\left(t V_{0}\right) \in M$ contains no cut point. In particular, this path is one to one and the point $\left(m_{0}, t V_{0}\right)$ remains in the neighborhood $\mathcal{N}_{\text {conj }}$ of the zero section of $T M$. Along the geodesic $\gamma_{0}$, we may thus take a Fermi chart $x$ as the one constructed above and use the estimates of Proposition 7.1 and 7.2, including (7.15) and (7.16).

We first calculate the components of the gradient of the test function $\phi_{1}$ in the Fermi chart $x$ and get:

$$
\operatorname{grad} \phi_{1}=\left(\partial_{j} f_{1}+D_{k} f_{1}\left(\partial_{j} g^{k \ell} \partial_{\ell} u+g^{k \ell} \partial_{j \ell} u\right)\right) g^{i j} \partial_{i},
$$

where the $g^{k \ell}(x)$ stand for the components of the matrix inverse of that of the Riemannian metric $g$, that is, where $g^{k \ell} g_{\ell i}=\delta_{i}^{k}$. Letting $x=0$, we find that the critical condition $\operatorname{grad} \phi_{1}\left(m_{0}\right)=0$ reads:

$$
\partial_{j} f_{1}+\sum_{k=1}^{2} D_{k} f_{1} \partial_{j k} u(0)=0, j=1,2,
$$

with the derivatives of $f_{1}$ evaluated at the point $(x, v, t)=\left(0,0,0, r_{0}, 1\right)$. The estimate $(7.15 \mathrm{a})$ prompts us to recast these equations as follows:

$$
\begin{aligned}
& \partial_{12} u(0)=a_{12} \partial_{11} u(0)+b_{12}, \\
& \partial_{22} u(0)=a_{22} \partial_{11} u(0)+b_{22},
\end{aligned}
$$

with:

$a_{12}=-\frac{D_{1} f_{1}}{D_{2} f_{1}}, b_{12}=-\frac{\partial_{1} f_{1}}{D_{2} f_{1}},, a_{22}=\left(\frac{D_{1} f_{1}}{D_{2} f_{1}}\right)^{2}, b_{22}=-\frac{\partial_{2} f_{1}}{D_{2} f_{1}}+\frac{\left(D_{1} f_{1}\right)\left(\partial_{1} f_{1}\right)}{\left(D_{2} f_{1}\right)^{2}}$.

By Proposition 7.1, we infer from $(7.18 \mathrm{a})(7.18 \mathrm{~b})$ the existence of a universal constant $C$ such that:

$$
\left|\partial_{12} u(0)\right| \leqslant C \varepsilon_{K}\left(1+\partial_{11} u(0)\right),\left|\partial_{22} u(0)\right| \leqslant C \varepsilon_{K}^{2}\left(1+\partial_{11} u(0)\right) .
$$

Differentiating once more the test function $\phi_{1}$ in the Fermi chart $x$, we find that the minimum condition $0 \leqslant d F[u]\left(\phi_{1}\right)\left(m_{0}\right)$ reads as follows:

$$
0 \leqslant I+I I+I I I+I V+V
$$


with:

$$
\begin{aligned}
I & =\sum_{i, j=1}^{2} \mathcal{F}_{j}^{i}(0) \partial_{i j} f_{1}, \\
I I & =r_{0} \sum_{i, j, k=1}^{2} \mathcal{F}_{j}^{i}(0)\left(D_{k} f_{1}\right) \partial_{i j} g^{k 2}(0), \\
I I I & =2 \sum_{i, j, k=1}^{2} \mathcal{F}_{j}^{i}(0)\left(D_{k} \partial_{j} f_{1}\right) \partial_{i k} u(0), \\
I V & =\sum_{i, j, k, \ell=1}^{2} \mathcal{F}_{j}^{i}(0)\left(D_{k \ell} f_{1}\right) \partial_{i k} u(0) \partial_{j \ell} u(0), \\
V & =\sum_{i, j, k=1}^{2} \mathcal{F}_{j}^{i}(0)\left(D_{k} f_{1}\right) \partial_{i j k} u(0),
\end{aligned}
$$

where the derivatives of $f_{1}$ are still evaluated at $(x, v, t)=\left(0,0,0, r_{0}, 1\right)$.

Combining (4.1) with Remark 4.1 and (7.8), we know that the potential function $u$ satisfies at $x=0$ the equation:

$$
\delta_{0} \operatorname{det}\left(\mathcal{H}_{i}^{j}(0)\right)=\frac{\rho_{0}(0,0)}{\rho_{t}\left(0, r_{0}\right)}
$$

with the matrix $\mathcal{H}_{i}^{j}(0)$ symmetric positive definite, given by

$$
\mathcal{H}_{i}^{j}(0)=\left(\begin{array}{cc}
\frac{1}{\delta_{0}} f_{0}\left(0,0,0, r_{0}, 1\right)+\partial_{11} u(0) & \partial_{12} u(0) \\
\partial_{12} u(0) & 1+\partial_{22} u(0)
\end{array}\right) .
$$

The positive definiteness of that matrix combined with (7.16a) implies that the minimum $\delta_{0}$ under study satisfies the inequality:

$$
\delta_{0} \geqslant \frac{1-C \varepsilon_{K}}{C+\partial_{11} u(0)}
$$

The latter shows that we may suppose $\partial_{11} u(0)$ larger than any suitable constant under control: for the moment, we take it larger than 1. Accordingly, we will freely replace $1+\partial_{11} u(0)$ merely by $\partial_{11} u(0)$ in the estimates $(7.18 \mathrm{c})$. Here, we should warn the reader that plugging the expressions (7.18) of $\partial_{12} u(0)$ and $\partial_{22} u(0)$ into (7.20a) yields for $\partial_{11} u(0)$ an affine equation which, by itself, provides no controlled upper bound on $\partial_{11} u(0)$.

We will require below the expressions at $x=0$ of two further matrices, namely:

$$
\mathcal{J}_{j}^{i}\left(0,0,0, r_{0}\right)=\left(\begin{array}{cc}
\frac{1}{\delta_{0}} f_{0}\left(0,0,0, r_{0}, 1\right) & 0 \\
0 & 1
\end{array}\right),
$$

which is known from (7.8), and:

$$
\mathcal{F}_{j}^{i}(0)=\frac{\rho_{t}\left(0, r_{0}\right)}{\rho_{0}(0,0)}\left(\begin{array}{cc}
\delta_{0}\left(1+\partial_{22} u(0)\right) & -\delta_{0} \partial_{12} u(0) \\
-\delta_{0} \partial_{12} u(0) & f_{0}\left(0,0,0, r_{0}, 1\right)+\delta_{0} \partial_{11} u(0)
\end{array}\right),
$$


obtained from (7.20) by inversion.

\subsubsection{Second order terms calculations}

In this section, we will calculate successively each of the terms $I$ to $I V$ which appear in the minimum condition (7.19), making use of the equations (7.17)(7.20a), the estimates (7.18c) and the expressions (7.20b)(7.22)(7.23). For short, we will not specify repeatedly that the derivatives of the functions $f_{1}, f_{0}$ are evaluated at the point $(x, v, t)=\left(0,0,0, r_{0}, 1\right)$; it will be implicitly understood throughout the calculations.

The term $I$ We calculate the term $I$ using (7.23) and find:

$$
\begin{aligned}
I=\frac{\rho_{t}\left(0, r_{0}\right)}{\rho_{0}(0,0)}\left(\delta_{0}\left(1+\partial_{22} u(0)\right) \partial_{11} f_{1}\right. & \\
& \left.\quad-2 \delta_{0} \partial_{12} u(0) \partial_{12} f_{1}-\left(f_{0}+\delta_{0} \partial_{11} u(0)\right) \partial_{22} f_{1}\right) .
\end{aligned}
$$

Recalling Proposition 7.1 and the estimates (7.16a)(7.18c), we infer the existence of a universal constant $C$ such that the following upper bound holds:

$$
I \leqslant C \frac{\rho_{t}\left(0, r_{0}\right)}{\rho_{0}(0,0)}\left(\varepsilon_{K}+\delta_{0} \partial_{11} u(0)+\delta_{0}\left(1+\partial_{22} u(0)\right)\right) .
$$

The term $I I$ Direct calculation yields for the term $I I$ the expression: $I I=-r_{0} D_{2} f_{1} \mathcal{F}_{1}^{1}(0) \partial_{11} \mathfrak{G}(0)$. Noting that $\partial_{11} \mathfrak{G}(0)=-2 K(0)$, we infer that $\partial_{11} \mathfrak{G}(0)$ is negative. Furthermore, by (7.15a), taking $\varepsilon_{K}+\delta_{0}$ smaller than a universal positive constant, we may consider that $D_{2} f_{1}\left(0,0,0, r_{0}, 1\right)$ is negative. The whole term $I I$ is thus negative and, as such, negligible in the minimum condition (7.19).

The term III Using (6.5a), the term III becomes:

$$
I I I=2 \sum_{k=1}^{2} D_{k} \partial_{k} f_{1}-2 \sum_{i, j, k=1}^{2} \mathcal{F}_{j}^{i}(0) \mathcal{J}_{i}^{k}\left(0,0,0, r_{0}\right) D_{k} \partial_{j} f_{1},
$$

hence the combination of (7.23) with (7.22) yields:

$$
\begin{aligned}
I I I & =2 \sum_{k=1}^{2} D_{k} \partial_{k} f_{1}-2 \frac{\rho_{t}\left(0, r_{0}\right)}{\rho_{0}(0,0)}\left\{f_{0}\left(1+\partial_{22} u(0)\right) D_{1} \partial_{1} f_{1}\right. \\
& \left.-f_{0} \partial_{12} u(0) D_{1} \partial_{2} f_{1}-\delta_{0} \partial_{12} u(0) D_{2} \partial_{1} f_{1}+\left(f_{0}+\delta_{0} \partial_{11} u(0)\right) D_{2} \partial_{2} f_{1}\right\} .
\end{aligned}
$$

From Proposition 7.1 and the estimates (7.16a)(7.18c), we infer the existence of a universal constant $C$ such that the following upper bound holds:

$$
I I I \leqslant C+C \frac{\rho_{t}\left(0, r_{0}\right)}{\rho_{0}(0,0)}\left(\delta_{0} \partial_{11} u(0)+1+\partial_{22} u(0)+\varepsilon_{K}\left|\partial_{12} u(0)\right|\right) .
$$


The term $I V$ Using (6.5b), the term $I V$ may be recast as follows:

$$
I V=\sum_{k, \ell=1}^{2}\left(\mathcal{H}_{\ell}^{k}(0)-2 \mathcal{J}_{\ell}^{k}+\sum_{i, j=1}^{2} \mathcal{F}_{j}^{i}(0) \mathcal{J}_{i}^{k} \mathcal{J}_{\ell}^{j}\right) D_{k \ell} f_{1},
$$

where the components of the Jacobi endomorphism field $\mathcal{J}$ are evaluated at the point $(x, v)=\left(0,0,0, r_{0}\right)$. Combining (7.20b) with (7.22) and (7.23), we find this term equal to:

$$
\begin{aligned}
\left(\partial_{11} u(0)+\frac{\rho_{t}\left(0, r_{0}\right)}{\delta_{0} \rho_{0}(0,0)} f_{0}^{2}\left(1+\partial_{22} u(0)\right)-\frac{f_{0}}{\delta_{0}}\right) D_{11} f_{1} \\
\quad+2 \partial_{12} u(0)\left(1-\frac{\rho_{t}\left(0, r_{0}\right)}{\rho_{0}(0,0)} f_{0}\right) D_{12} f_{1} \\
\quad+\left(\delta_{0} \frac{\rho_{t}\left(0, r_{0}\right)}{\rho_{0}(0,0)} \partial_{11} u(0)+\partial_{22} u(0)+\frac{\rho_{t}\left(0, r_{0}\right)}{\rho_{0}(0,0)} f_{0}-2\right) D_{22} f_{1}
\end{aligned}
$$

From $(7.15 \mathrm{c}),(7.15 \mathrm{~d})$ and $(7.16 \mathrm{a})$, we may take for granted at the point $(x, v, t)=\left(0,0,0, r_{0}, 1\right)$ the inequalities:

$$
D_{11} f_{1} \leqslant-\frac{1}{2 \pi^{2}},-\frac{3}{4} \leqslant f_{0} \leqslant-\frac{1}{2}, \frac{1}{\pi^{2}} \leqslant D_{22} f_{1} \leqslant \frac{3}{\pi^{2}},
$$

provided $\varepsilon_{K}+\delta_{0}$ is assumed smaller than a universal positive constant. The combination of Proposition 7.1 with (7.18) thus implies the existence of a universal constant $C$ such that the following upper bound holds:

$$
\begin{aligned}
I V \leqslant & -\frac{1}{2 \pi^{2}}\left(\partial_{11} u(0)+\frac{\rho_{t}\left(0, r_{0}\right)}{4 \delta_{0} \rho_{0}(0,0)}\left(1+\partial_{22} u(0)\right)+\frac{1}{2 \delta_{0}}\right) \\
& +C \varepsilon_{K}^{2} \partial_{11} u(0)+\frac{\rho_{t}\left(0, r_{0}\right)}{\rho_{0}(0,0)}\left(C \varepsilon_{K}\left|\partial_{12} u(0)\right|+\frac{3}{\pi^{2}} \delta_{0} \partial_{11} u(0)\right)
\end{aligned}
$$

The terms appearing in the first part of the right-hand side, all large negative for $\delta_{0}>0$ small enough, are the ones that will make our proof of Estimate 1 work.

\subsubsection{Third order terms calculations}

In this section, we will calculate the term $V$ of the minimum condition (7.19). To do so, we cannot use directly the equations $(7.17)(7.20 \mathrm{a})$, the estimates (7.18c) and the expressions $(7.20 \mathrm{~b})(7.22)(7.23)$, as done for the preceding terms, because the term $V$ is a linear combination of third derivatives of the potential function $u$. We will overcome this difficulty by using the continuity equation (4.1), differentiated once, to derive an expression of the term $V$ involving only lower order derivatives of $u$. We will then complete as above the calculation of that expression. 
Differentiating the continuity equation at the minimum point We first describe a convenient way of writing the continuity equation near the minimum point $m_{0}$. Let $x \mapsto\left(\zeta_{1}(x), \zeta_{2}(x)\right)$ be the local direct orthonormal frame field given in the Fermi chart by:

$$
\begin{aligned}
\zeta_{2}=\frac{\operatorname{grad} u}{|\operatorname{grad} u|}=\frac{1}{|\operatorname{grad} u|} & \left(\partial_{1} u \partial_{1}+\frac{1}{\mathfrak{G}} \partial_{2} u \partial_{2}\right), \\
& \text { and thus: } \zeta_{1}=\frac{1}{\sqrt{\mathfrak{G}}|\operatorname{grad} u|}\left(\partial_{2} u \partial_{1}-\partial_{1} u \partial_{2}\right) .
\end{aligned}
$$

Setting $\mathcal{H}\left(\zeta_{i}\right)=H_{i}^{j} \zeta_{j}$, we claim that the continuity equation (4.1), explicited as in Remark 4.1, reads in the Fermi chart as follows:

$$
\phi_{1}(x) \operatorname{det}\left(H_{i}^{j}(x)\right)=\frac{\rho_{0}(x)}{\rho_{t}\left(G_{u}(x)\right)}
$$

with:

$$
H_{i}^{j}(0)=\left(\begin{array}{cc}
\frac{f_{0}}{f_{1}}+\nabla d u\left(\zeta_{1}, \zeta_{1}\right) & \nabla d u\left(\zeta_{1}, \zeta_{2}\right) \\
\nabla d u\left(\zeta_{2}, \zeta_{1}\right) & \mathfrak{G}(x)+\nabla d u\left(\zeta_{2}, \zeta_{2}\right)
\end{array}\right)
$$

where the functions $f_{0}$ and $f_{1}$ are evaluated at the point:

$$
(x, v, t)=(x, \operatorname{grad} u(x), 1)=\left(x^{1}, x^{2}, \partial_{1} u(x), \frac{1}{\mathfrak{G}(x)} \partial_{2} u(x), 1\right)
$$

and $G_{u}(x)$ stands for simplicity for the local expression of the gradientmapping $G_{u}$, equal to $X\left(x^{1}, x^{2}, \partial_{1} u(x), \frac{1}{\mathfrak{G}(x)} \partial_{2} u(x), 1\right)$. Indeed, the claim is straightforward at $x=0$ and it can be checked elsewhere by using the Fermi chart constructed there similarly to ours at $x=0$.

Taking the logarithm of both sides of (7.27) and differentiating the resulting equation at $x=0$ (where the test function $\phi_{1}$ assumes its minimum $\delta_{0}$ ), we obtain the 1 -forms equality:

$$
\mathcal{F}_{j}^{i}(0) d H_{i}^{j}(0)=\frac{d \rho_{0}}{\rho_{0}}(0,0)-\frac{d \rho_{t}}{\rho_{t}}\left(0, r_{0}\right) \circ d G_{u}(0)
$$

Let us proceed to expressing this equation in the Fermi chart. Here, and in the sequel of this subsection, it is implicitly understood that the functions $f_{0}, f_{1}$ and their derivatives are evaluated at the point $(x, v, t)=\left(0,0,0, r_{0}, 1\right)$. In particular, the function $f_{1}$ itself is equal to $\delta_{0}$. From (7.14), we already know that the matrix of the endomorphism $d G_{u}(0)$ in the Fermi chart is equal to $\left(\begin{array}{cc}f_{0}+\delta_{0} \partial_{11} u(0) & \delta_{0} \partial_{12} u(0) \\ \partial_{21} u(0) & 1+\partial_{22} u(0)\end{array}\right)$. Let us calculate the components of the 
$d H_{i}^{j}(0)$ using the vanishing of $d \mathfrak{G}(0)$ and $d \phi_{1}(0)$. We get successively:

$$
\begin{aligned}
& \partial_{1} H_{1}^{1}(0)=\frac{\partial_{1} f_{0}}{\delta_{0}}-\frac{2}{r_{0}} \partial_{11} u(0) \partial_{12} u(0)+\partial_{111} u(0), \\
& \partial_{1} H_{1}^{2}(0)=\partial_{1} H_{2}^{1}(0)=r_{0} K(0)+\frac{1}{r_{0}} \partial_{11} u(0)\left(\partial_{11} u(0)-\partial_{22} u(0)\right)+\partial_{112} u(0), \\
& \partial_{1} H_{2}^{2}(0)=\frac{2}{r_{0}} \partial_{11} u(0) \partial_{12} u(0)+\partial_{122} u(0), \text { and: } \\
& \partial_{2} H_{1}^{1}(0)=\frac{\partial_{2} f_{0}}{\delta_{0}}-\frac{2}{r_{0}}\left(\partial_{12} u(0)\right)^{2}+\partial_{112} u(0), \\
& \partial_{2} H_{1}^{2}(0)=\partial_{2} H_{2}^{1}(0)=\frac{1}{r_{0}} \partial_{12} u(0)\left(\partial_{11} u(0)-\partial_{22} u(0)\right)+\partial_{122} u(0), \\
& \partial_{2} H_{2}^{2}(0)=\frac{2}{r_{0}}\left(\partial_{12} u(0)\right)^{2}+\partial_{222} u(0) .
\end{aligned}
$$

Let us plug in (7.28) these expressions of $d G_{u}(0)$ and the $d H_{i}^{j}(0)$, together with those of the $\mathcal{F}_{j}^{i}(0)$ given by (7.23). Solving each resulting equation for the third order terms, we find:

$$
\begin{aligned}
& \sum_{i, j=1}^{2} \mathcal{F}_{j}^{i}(0) \partial_{1 i j} u(0)=\frac{\partial_{1} \rho_{0}}{\rho_{0}}-\frac{1}{\rho_{t}}\left(\partial_{1} \rho_{t}\left(f_{0}+\delta_{0} \partial_{11} u\right)+\partial_{2} \rho_{t} \partial_{12} u\right) \\
& \quad+\frac{\rho_{t}}{\rho_{0}}\left(-\partial_{1} f_{0}-D_{1} f_{0} \partial_{11} u+\left(2 \delta_{0} r_{0} K(0)-D_{2} f_{0}\right) \partial_{12} u-\partial_{1} f_{0} \partial_{22} u\right. \\
& \left.\quad+\frac{2}{r_{0}}\left(\delta_{0}-f_{0}\right) \partial_{11} u \partial_{12} u-D_{1} f_{0} \partial_{11} u \partial_{22} u-D_{2} f_{0} \partial_{12} u \partial_{22} u\right), \quad(7.29 a) \\
& \sum_{i, j=1}^{2} \mathcal{F}_{j}^{i}(0) \partial_{2 i j} u(0)=\frac{\partial_{2} \rho_{0}}{\rho_{0}}-\frac{1}{\rho_{t}}\left(\delta_{0} \partial_{1} \rho_{t} \partial_{12} u+\partial_{2} \rho_{t}\left(1+\partial_{22} u\right)\right) \\
& +\frac{\rho_{t}}{\rho_{0}}\left(-\partial_{2} f_{0}-D_{1} f_{0} \partial_{12} u-\left(\partial_{2} f_{0}+D_{2} f_{0}\right) \partial_{22} u+\frac{2}{r_{0}}\left(\delta_{0}-f_{0}\right)\left(\partial_{12} u\right)^{2}\right. \\
& \left.-D_{1} f_{0} \partial_{12} u \partial_{22} u-D_{2} f_{0}\left(\partial_{22} u\right)^{2}\right), \quad(7.29)
\end{aligned}
$$

where, of course, the prescribed density functions $\rho_{0}, \rho_{t}$ and their first partial derivatives are evaluated respectively at $x=0$ and $x=\left(0, r_{0}\right)$, while the second partial derivatives of the potential function $u$ are evaluated at $x=0$. Remarkably, in the process of this calculation, the cubic terms in $\partial^{2} u$ which the reader would expect to appear in (7.29) have cancelled.

The term $V$ With (7.29) at hand, we are ready to calculate the term $V=$ $\sum_{k=1}^{2} D_{k} f_{1}\left(\sum_{i, j=1}^{2} \mathcal{F}_{j}^{i}(0) \partial_{i j k} u(0)\right)$. Here, three simplifications occur based on the combination of the critical conditions (7.17) with (7.29). Indeed, 
on the one hand, we gather the terms in $\partial^{2} u$ involving the gradient of the prescribed density function $\rho_{t}$, namely:

$$
-\frac{1}{\rho_{t}}\left(\delta_{0} \partial_{1} \rho_{t}\left(D_{1} f_{1} \partial_{11} u+D_{2} f_{1} \partial_{12} u\right)+\partial_{2} \rho_{t}\left(D_{1} f_{1} \partial_{12} u+D_{2} f_{1} \partial_{22} u\right)\right)
$$

and, using (7.17), we find their sum equal to:

$$
\frac{1}{\rho_{t}}\left(\delta_{0} \partial_{1} \rho_{t} \partial_{1} f_{1}+\partial_{2} \rho_{t} \partial_{2} f_{1}\right)
$$

On the other hand, we gather the quadratic terms in $\partial^{2} u$, namely:

$$
\begin{aligned}
& \frac{\rho_{t}}{\rho_{0}}\left(\frac{2}{r_{0}}\left(\delta_{0}-f_{0}\right)\left(D_{1} f_{1} \partial_{11} u+D_{2} f_{1} \partial_{12} u\right) \partial_{12} u\right. \\
& \left.-\left(D_{1} f_{0}\left(D_{1} f_{1} \partial_{11} u+D_{2} f_{1} \partial_{12} u\right)+D_{2} f_{0}\left(D_{1} f_{1} \partial_{12} u+D_{2} f_{1} \partial_{22} u\right)\right) \partial_{22} u\right)
\end{aligned}
$$

and find, by (7.17) again, that their sum becomes linear in $\partial^{2} u$, equal to:

$$
\frac{\rho_{t}}{\rho_{0}}\left(-\frac{2}{r_{0}}\left(\delta_{0}-f_{0}\right) \partial_{1} f_{1} \partial_{12} u+\left(D_{1} f_{0} \partial_{1} f_{1}+D_{2} f_{0} \partial_{2} f_{1}\right) \partial_{22} u\right) .
$$

Finally, we gather the terms in $D_{j} f_{0} D_{k} f_{1} \partial_{j k} u$ and get similarly:

$$
-\frac{\rho_{t}}{\rho_{0}} \sum_{j, k=1}^{2} D_{j} f_{0} D_{k} f_{1} \partial_{j k} u=\frac{\rho_{t}}{\rho_{0}} \sum_{j=1}^{2} D_{j} f_{0} \partial_{j} f_{1} .
$$

Altogether, we obtain for the term $V$ the following simplified expression:

$$
\begin{aligned}
V= & D_{1} f_{1}\left(\frac{\partial_{1} \rho_{0}}{\rho_{0}}-\frac{\partial_{1} \rho_{t}}{\rho_{t}} f_{0}\right)+D_{2} f_{1}\left(\frac{\partial_{2} \rho_{0}}{\rho_{0}}-\frac{\partial_{2} \rho_{t}}{\rho_{t}}\right) \\
+ & \frac{\rho_{t}}{\rho_{0}}\left(D_{1} f_{0} \partial_{1} f_{1}-D_{1} f_{1} \partial_{1} f_{0}+D_{2} f_{0} \partial_{2} f_{1}-D_{2} f_{1} \partial_{2} f_{0}\right)\left(1+\partial_{22} u\right) \\
& +\frac{\rho_{t}}{\rho_{0}}\left(2 \delta_{0} r_{0} K(0) D_{1} f_{1}-\frac{2}{r_{0}}\left(\delta_{0}-f_{0}\right) \partial_{1} f_{1}\right) \partial_{12} u
\end{aligned}
$$

Combining Propositions 7.1 and 7.2, we infer the existence of a universal constant $C$ such that the following upper bound holds:

$$
\begin{aligned}
V \leqslant C\left(\max _{M}\left|d \log \rho_{0}\right|\right. & \left.+\max _{M}\left|d \log \rho_{t}\right|\right) \\
& +C \frac{\rho_{t}\left(0, r_{0}\right)}{\rho_{0}}\left(\varepsilon_{K}\left(1+\partial_{22} u(0)\right)+\varepsilon_{K}\left|\partial_{12} u(0)\right|\right) .
\end{aligned}
$$




\subsubsection{Minimum condition: final discussion}

Let us sum up the main reductions operated so far at the minimum point $m_{0}$ of the function $\phi_{1}$. We know the existence of two universal positive constants, $C$ (large) and $\iota$ (small), such that, if the geometric parameter $\varepsilon_{K}=|K-1|_{C^{2}(M)}$ is smaller than $\iota$, either the positive minimum under study $\delta_{0}=\min _{M} \phi_{1}$ is bounded below by $\iota$, in which case we are done, or if not, the length $r_{0}$ of the vector $V_{0}=\operatorname{grad} u\left(m_{0}\right)$ must be larger than $\frac{3 \pi}{4}$ (due to $(7.11)$ read at $t=1$ ). Moreover, in the Fermi chart constructed along the geodesic segment $\gamma_{0}(t)=\exp _{m_{0}}\left(t V_{0}\right), t \in[0,1]$, the second partial derivative $\partial_{11} u(0)$ must be larger than 1 (due to (7.21)) and the following inequality holds:

$$
\begin{aligned}
0 \leqslant-\frac{1}{2 \pi^{2}} & \left(\partial_{11} u(0)+\frac{\rho_{t}\left(0, r_{0}\right)}{4 \delta_{0} \rho_{0}(0,0)}\left(1+\partial_{22} u(0)\right)+\frac{1}{2 \delta_{0}}\right) \\
& +C \varepsilon_{K}^{2} \partial_{11} u(0)+C\left(\max _{M}\left|d \log \rho_{0}\right|+\max _{M}\left|d \log \rho_{t}\right|\right) \\
& +C \frac{\rho_{t}\left(0, r_{0}\right)}{\rho_{0}(0,0)}\left(\delta_{0} \partial_{11} u(0)+1+\partial_{22} u(0)+\varepsilon_{K}\left|\partial_{12} u(0)\right|\right) .
\end{aligned}
$$

This inequality is obtained from the minimum condition (7.19) after plugging in the upper bounds derived in sections 7.2.3 and 7.2.4, namely: $I I \leqslant 0$ and (7.24)(7.25)(7.26)(7.30). To discuss (7.31) complying with the statement of Theorem 1.4, we should beware that the size of the parameter $\varepsilon_{K}$ must stay independent of the given densities $\rho_{0}, \rho_{1}$. We thus fix the value of $\varepsilon_{K}$ in $(7.31)$ once for all equal to $\min \left(\iota, \frac{1}{6 C \pi^{2}}\right)$. This choice, combined with (7.31), yields the inequality:

$$
\begin{gathered}
0 \leqslant \partial_{11} u(0)\left(\delta_{0} C \frac{\max _{M} \rho_{t}}{\min _{M} \rho_{0}}-\frac{1}{6 \pi^{2}}\right)+\frac{\rho_{t}\left(0, r_{0}\right)}{\rho_{0}(0,0)}\left(1+\partial_{22} u(0)\right)\left(C-\frac{1}{16 \pi^{2} \delta_{0}}\right) \\
+\left(C\left(\max _{M}\left|d \log \rho_{0}\right|+\max _{M}\left|d \log \rho_{t}\right|+\frac{\max _{M} \rho_{t}}{\min _{M} \rho_{0}}\right)-\frac{1}{4 \pi^{2} \delta_{0}}\right) \\
+\left(\frac{\rho_{t}\left(0, r_{0}\right)}{\rho_{0}(0,0)}\left(C\left|\partial_{12} u(0)\right|-\frac{1}{16 \pi^{2} \delta_{0}}\left(1+\partial_{22} u(0)\right)\right)-\frac{1}{6 \pi^{2}} \partial_{11} u(0)\right), \quad \text { (7.32) }
\end{gathered}
$$

where we have intentionally split the right-hand side into four pieces. Let us focus provisionaly on the last one. We require the pinching:

$$
\frac{\pi}{2} \leqslant \frac{1}{\left|D_{2} f_{1}\right|} \leqslant 2 \pi
$$

which is valid due to (7.15a) and the smallness of the constant $\iota$. Here and below, the derivatives of the function $f_{1}$ are still understood evaluated at the point $(x, v, t)=\left(0,0,0, r_{0}, 1\right)$. On the one hand, from (7.18a) combined with Proposition (7.1) and (7.33), we may write: $\left|\partial_{12} u(0)\right| \leqslant 2 \pi\left|D_{1} f_{1}\right| \partial_{11} u(0)+$ 
$C \varepsilon_{K}$. On the other hand, from (7.18b) combined with Proposition (7.1) and (7.33), the lower bound: $1+\partial_{22} u(0) \geqslant \frac{\pi^{2}}{4}\left|D_{1} f_{1}\right|^{2} \partial_{11} u(0)$ holds. The last piece under study is thus bounded above by:

$$
\partial_{11} u(0)\left[\frac{\max _{M} \rho_{t}}{\min _{M} \rho_{0}}\left|D_{1} f_{1}\right|\left(2 \pi C-\frac{1}{64 \delta_{0}}\left|D_{1} f_{1}\right|\right)-\frac{1}{6 \pi^{2}}\right]+C \frac{\max _{M} \rho_{t}}{\min _{M} \rho_{0}},
$$

hence also by:

$$
\partial_{11} u(0)\left[\delta_{0} 64 \pi^{2} C^{2} \frac{\max _{M} \rho_{t}}{\min _{M} \rho_{0}}-\frac{1}{6 \pi^{2}}\right]+C \frac{\max _{M} \rho_{t}}{\min _{M} \rho_{0}} .
$$

Replacing by the latter expression the last piece of the right-hand side of (7.32), we obtain the inequality:

$$
\begin{aligned}
0 \leqslant \partial_{11} u(0)\left[\delta_{0}(C\right. & \left.\left.+64 \pi^{2} C^{2}\right) \frac{\max _{M} \rho_{t}}{\min _{M} \rho_{0}}-\frac{1}{3 \pi^{2}}\right] \\
& +\frac{\rho_{t}\left(0, r_{0}\right)}{\rho_{0}(0,0)}\left(1+\partial_{22} u(0)\right)\left[C-\frac{1}{16 \pi^{2} \delta_{0}}\right] \\
+ & {\left[C\left(\max _{M}\left|d \log \rho_{0}\right|+\max _{M}\left|d \log \rho_{t}\right|+2 \frac{\max _{M} \rho_{t}}{\min _{M} \rho_{0}}\right)-\frac{1}{4 \pi^{2} \delta_{0}}\right] }
\end{aligned}
$$

from which we conclude that $\delta_{0}$, or else $\min _{M} \operatorname{Jac}\left(\exp _{m}\right)\left(\operatorname{grad} u_{t}(m)\right)$, must be bounded below by:

$$
\begin{aligned}
\min _{t \in[0,1]}\left(\iota, \frac{1}{16 \pi^{2} C}\right. & , \frac{\min _{M} \rho_{0}}{3 \pi^{2} C\left(1+64 \pi^{2} C\right) \max _{M} \rho_{t}}, \\
& \left.\frac{1}{4 \pi^{2} C\left(\max _{M}\left|d \log \rho_{0}\right|+\max _{M}\left|d \log \rho_{t}\right|+2 \frac{\max _{M} \rho_{t}}{\min _{M} \rho_{0}}\right)}\right) .
\end{aligned}
$$

This minimum is therefore bounded below by the constant $\delta>0$ defined by:

$$
\delta=\min \left(\iota, \frac{1}{16 \pi^{2} C}, \frac{e^{-2 \mathfrak{M}_{1}}}{3 \pi^{2} C\left(1+64 \pi^{2} C\right)}, \frac{1}{4 \pi^{2} C\left(2 \mathfrak{M}_{1}+e^{2 \mathfrak{M}_{1}}\right)}\right),
$$

with $\mathfrak{M}_{1}=\max _{t \in[0,1]}\left|\log \rho_{t}\right|_{C^{1}(M)}$. This constant is, indeed, under control in the sense of Remark 1.1. The proof of Estimate 1, in the case of Theorem 1.4 , is thus complete

\section{A Assumptions comparison: curvature closeness versus metric closeness}

So far, two different assumptions bearing on the Riemannian metric $g$ of $\mathbb{S}^{2}$ have been used to study the regularity of the optimal transport potential 
function: a metric closeness assumption [FR09, Theorem 1.2] [FRV10, Theorem 5.1] and the curvature closeness assumption used in Theorem 1.4 and in [DG11, Theorems 1 and 2]. They go respectively as follows:

(i) the metric $g$ is $C^{4}$ close to a Riemannian metric $g_{0}$ of constant Gauss curvature, say, equal to 1 ;

(ii) the Gauss curvature of $g$ is $C^{2}$ close to 1 .

The distinction between these two assumptions is tacitly ignored ${ }^{6}$ in [FR09, bottom p.1695, top p.1696] [FRV11, p.872, section 7]. To clarify this topic, we will prove that conditions $(i)$ and $(i i)$ are not equivalent. The former implies the latter, but the converse is not true. Here is an example:

Lemma A.1. Let $g_{0}$ be a metric on $\mathbb{S}^{2}$ with constant Gauss curvature $K_{0}=1$. There exists a sequence $\left(g_{i}\right)_{i \in \mathbb{N}^{*}}$ of metrics on $\mathbb{S}^{2}$, pointwise conformal to $g_{0}$, converging to $g_{0}$ in $C^{3, \alpha}$ norm, for every $\alpha \in(0,1)$, but not in $C^{4}$ norm, and such that the corresponding sequence of Gauss curvature functions $\left(K_{i}\right)_{i \in \mathbb{N}^{*}}$ converges to 1 in $C^{2}$ norm.

Proof. Working with the metric $g_{0}$ and setting $\Delta_{0}$ for its positive Laplacian, take a function $f: \mathbb{S}^{2} \rightarrow \mathbb{R}$ of zero average, twice continuously differentiable and not better, and such that the solution $\varphi: \mathbb{S}^{2} \rightarrow \mathbb{R}$ of the Poisson equation $\Delta_{0} \varphi=f$ is not $C^{4}$. Set $p_{1}$ for the orthogonal projection of $L^{2}$ on the eigenspace $\Lambda_{1}$ associated to the first positive eignevalue of $\Delta_{0}$, and $u \mapsto \mathcal{K}[u]=e^{-2 u}\left(\Delta_{0} u+1\right)$, for the conformal Gaussian curvature operator. For every $\alpha \in(0,1)$, the map:

$$
u \in C^{3, \alpha}\left(\mathbb{S}^{2}\right) \rightarrow F[u]=\mathcal{K}[u]+p_{1}(u) \in C^{1, \alpha}\left(\mathbb{S}^{2}\right)
$$

is locally invertible near $u=0$ [Del03, p.35] hence, for each small enough real $t$, there exists a unique function $u_{t}$ close to 0 in $C^{3, \alpha}\left(\mathbb{S}^{2}\right)$, solving the equation $F\left[u_{t}\right]=1+t f$. The solution $u_{t}$ vanishes for $t=0$ and is differentiable with respect to $t$. We infer that its derivative $\dot{u}_{0}=\left.\frac{d u_{t}}{d t}\right|_{t=0}$ satisfies the equation: $\Delta_{0} \dot{u}_{0}-2 \dot{u}_{0}+p_{1}\left(\dot{u}_{0}\right)=f$, or else: $\Delta_{0}\left(\varphi-\dot{u}_{0}\right)=p_{1}\left(\dot{u}_{0}\right)-2 \dot{u}_{0}$, where the righthand side lies in $C^{3, \alpha}\left(\mathbb{S}^{2}\right)$. The Schauder estimates yield $\left(\varphi-\dot{u}_{0}\right) \in C^{5, \alpha}\left(\mathbb{S}^{2}\right)$. Claim: we can find a sequence $\left(t_{i}\right)_{i \in \mathbb{N}^{*}}$, with $t_{i} \in\left(0, \frac{1}{i}\right)$, such that, for each $i \in \mathbb{N}^{*}$, the function $u_{t_{i}}$ does not belong to $C^{4}\left(\mathbb{S}^{2}\right)$.

Indeed, by contradiction, if not, there exists $i \in \mathbb{N}^{*}$ such that all the functions $u_{t}$ belong to $C^{4}\left(\mathbb{S}^{2}\right)$ provided $t$ lies in $\left(0, \frac{1}{i}\right)$. If so, the limit of the ratio $\frac{u_{t}}{t}$ as $t \rightarrow 0$, equal to $\dot{u}_{0}$, must belong to $C^{4}\left(\mathbb{S}^{2}\right)$ as well. Recalling the above Schauder regularity result, we conclude that $\varphi \in C^{4}\left(\mathbb{S}^{2}\right)$, getting a contradiction. So the claim is verified.

\footnotetext{
${ }^{6}$ worse, the assumption quoted in [FR09, pp.1695-1696] is not the condition (ii) but a mere positive curvature pinching, namely the one required in [DG10, Theorem 1]
} 
By construction, the sequence of $C^{3, \alpha}\left(\mathbb{S}^{2}\right)$ conformal metrics defined by $g_{i}=e^{2 u_{t_{i}}} g_{0}$, for $i \in \mathbb{N}^{*}$, satisfies $\left|K_{i}-1\right|_{C^{2}} \equiv\left|t_{i} f-p_{1}\left(u_{t_{i}}\right)\right|_{C^{2}}$ hence $\lim _{i \rightarrow \infty}\left|K_{i}-1\right|_{C^{2}}=0$, while $\left|g_{i}-g_{0}\right|_{C^{4}}$ is infinite, which proves the lemma $\square$

Remark A.1. In the condition ( $i i)$ above, we did not mention the normalization $\min _{\mathbb{S}^{2}} K=1$ used in Theorem 1.4 because we did not care quantifying the closeness condition in a scale invariant fashion. In any case, given a metric $g$ with Gauss curvature $K$ sufficiently close to 1 in $C^{2}$ norm, one may consider the rescaled metric $g^{\prime}=g \min _{\mathbb{S}^{2}} K$. The Gauss curvature $K^{\prime}$ of $g^{\prime}$ satisfies the normalization $\min _{\mathbb{S}^{2}} K^{\prime}=1$ and one can readily verify that it is still $C^{2}$ close to 1 . The reader may wish to apply this remark, for $i$ large, to the metric $g_{i}$ just constructed. The resulting sequence of metrics will satisfy the conclusion of Lemma A.1 as well.

On the 2-sphere endowed with a Riemannian metric like ${ }^{7} g_{i}$ (for $i$ large), how can we tackle the smoothness issue in optimal transport? The continuity results [FR09, Theorem 1.2] and [FRV11, Theorem 1.3] combined with [FRV10, Theorem 5.1] cannot be applied because the metric is not $C^{4}$. We cannot apply Theorem 1.4, as stated, either. This difficulty motivates the following ad hoc extension of the results of the present paper:

Proposition A.1. If we allow the positive probability measures $\left(\mu_{0}, \mu_{1}\right)$ to be only $C^{2}$, the conclusions of Theorems 1.3 and 5.1 remain valid with $k=1$ and $\alpha \in(0,1)$ arbitrary. Moreover, if we let the Riemannian metric $g$ be only $C^{3}$, but require that its curvature tensor be $C^{2}$, keeping unchanged the other assumptions possibly made on the manifold (like having Gauss curvature $C^{2}$ close to 1, or like being an A3 manifold), the conclusions of Theorems 1.4, 1.5 and 6.1 remain valid with $k=1$ and $\alpha \in(0,1)$ arbitrary.

Proof. Let $M$ be a closed manifold endowed with a $C^{3}$ Riemannian metric $g$, the curvature tensor of which is $C^{2}$. By a standard result in ODE theory about regularity with respect to initial conditions [Car67, Har02], applied to the geodesic equation, the exponential map must be $C^{2}$. Actually, it is even $C^{3}$ because we have assumed that the curvature is $C^{2}$. Indeed, a standard result in ODE theory about regularity with respect to a parameter [Car67, Har02], applied to the Jacobi equation (see section 2.3.1), implies that the differential of the exponential map is $C^{2}$. In dimension 2 , it further implies that the functions $f_{0}$ and $f_{1}$ defined by (7.4) are $C^{2}$. Therefore one can still carry through the proof of [DG11, Theorem 2]. Moreover, in any dimension, the positive function $(m, V) \mapsto \operatorname{Jac}\left(\exp _{m}\right)(V) \in \mathbb{R}$ and the Jacobi endomorphism field (see section 2.1.3) are $C^{2}$ on the domain $\mathcal{N}_{\text {conj }} \subset T M$. In particular, it makes sense to say that the manifold $M$ is A3 (see Definition 6.1). Finally, given a couple $\left(\rho_{0}, \rho_{1}\right)$ of $C^{2}$ positive probability densities

\footnotetext{
${ }^{7}$ rescaled as just explained
} 
on the Riemannian manifold $(M, g)$, the real function $(m, V) \mapsto \psi_{t}(m, V)$ introduced in the $\log$ form of the continuity equation (4.2) is also $C^{2}$ on the domain $\mathcal{N}_{\text {conj. }}$. For every fixed Hölder exponent $\alpha \in(0,1)$, we may thus consider the operator $\Phi$ defined in section 4.1 as a mapping from $[0,1] \times \mathcal{A}^{3, \alpha}$ to $P_{1}^{1, \alpha}$. In this setting, all the arguments used in section 4 remain valid. Besides, keeping in mind the preceding smoothness observations, one can verify that the proofs given in section 7 remain fully applicable. As regards section 6 , though, the test function $\mathfrak{T}_{t}$ is not $C^{2}$ any more, but only $C^{1, \alpha}$. So we must justify the maximum inequality $(6.1 b)$ on which the proof of the Ma-Trudinger-Wang estimate given in section 6.2 is based. A similar difficulty arises at the start of the estimate of [Del82, pp.428-429] quoted in the proof of Theorem 5.1. Here, we will explain how it can be overcome in the case of section 6.2. One can proceed along the same lines to fix the proof of [Del82, pp.428-429], hence that of Theorem 5.1. The argument goes as follows.

For convenience, we set $\tilde{\psi}_{t}$ for the pullback on $M$ by the vector field $\operatorname{grad} u_{t}$ of the function $\psi_{t}$. The function $\tilde{\psi}_{t}$ is $C^{2}$. Dropping the subscript $t$, we claim that the function $\mathfrak{T}$ satisfies weakly on the manifold the equation $d F[u](\mathfrak{T})=Q[u]-\Delta \tilde{\psi}$, where the auxiliary differential operator $Q[u]$ is defined by:

$$
Q[u]:=\mathcal{F}_{j}^{i} D_{\ell} \mathcal{J}_{k}^{k} \nabla_{i}^{j} \nabla^{\ell} u+\mathcal{F}_{p}^{i} \mathcal{F}_{j}^{q} \nabla_{k} \mathcal{H}_{q}^{p} \nabla^{k} \mathcal{H}_{i}^{j}-\mathcal{F}_{j}^{i} D_{\ell} \mathcal{J}_{i}^{j} \nabla_{k}^{k} \nabla^{\ell} u+\text { l.o.t. }
$$

l.o.t. standing here for the lower order terms given by (6.4) minus those given by (6.7). Let us take the claim for granted and defer its proof. Importantly for us, the function $Q[u]-\Delta \tilde{\psi}$ is continuous. We want to prove that it is nonpositive at the point $m_{0}$ where the test function $\mathfrak{T}$ assumes its maximum. Let us argue by contradiction and suppose that $(Q[u]-\Delta \tilde{\psi})\left(m_{0}\right)>0$. If so, by continuity, the inequality $Q[u]-\Delta \tilde{\psi} \geqslant 0$ holds in a neighborhood of $m_{0}$. By the strong maximum principle for weak subsolutions [GT01, Chap. $8]$, the test function $\mathfrak{T}$ must be constant on that neighborhood. It follows that $Q[u]-\Delta \tilde{\psi}\left(m_{0}\right)=0$, hence a contradiction. Therefore the inequality $(Q[u]-\Delta \tilde{\psi})\left(m_{0}\right) \leqslant 0$ holds, indeed. Relying on this inequality, one may now carry through the proof given in section 6.2.

We are left with proving the above claim. Let $\left(u_{N}\right)_{N \in \mathbb{N}}$ be a sequence of $C^{4}$ functions converging in $C^{3}$ toward the potential function $u$. For $N$ large, the function $u_{N}$ is admissible and it is convenient to set $\mathcal{H}_{N}=\mathcal{H} u_{N}, \mathcal{T}_{N}=$ $\operatorname{trace}\left(\mathcal{H}_{N}\right), \mathcal{F}_{N}=\mathcal{F}_{\left[u_{N}\right]}, G_{N}=G_{u_{N}}, \rho_{N} d V_{g}=\left(G_{N}\right)_{\#} \mu_{0}, Q_{N}=Q\left[u_{N}\right]$ and $\tilde{\psi}_{N}=\log \rho_{0}-\log \left(\left(\rho_{N} \circ G_{N}\right) \operatorname{Jac}(\exp )\left(\operatorname{grad} u_{N}\right)\right)$. For later use, we note that, as $N$ goes to $\infty$, the function $Q_{N}$ tends to $Q[u]$ in $C^{0}$ while the tensor $\mathcal{F}_{N}$ and the trace $\mathcal{T}_{N}$ tend respectively to the tensor $\mathcal{F}_{[u]}$ and to the test function $\mathcal{T}=\operatorname{trace}(\mathcal{H} u)$ in $C^{1}$. Furthermore, the probability density $\rho_{N}$ and the function $\tilde{\psi}_{N}$ tend respectively to $\rho_{t}$ and to $\tilde{\psi}=\tilde{\psi}_{t}$ in $C^{1}$. Fixing an 
arbitrary $C^{1}$ function $v$, let us consider the integral $I_{v}\left[u_{N}\right]$ defined by:

$$
I_{v}\left[u_{N}\right]:=-\int_{M}\left(\left(\mathcal{F}_{N}\right)_{j}^{i} \nabla_{i} v+v \nabla_{i}\left(\mathcal{F}_{N}\right)_{j}^{i}\right) \nabla^{j} \mathcal{T}_{N} d V_{g}
$$

This integral is, by construction, equal to $\int_{M} v d F\left[u_{N}\right]\left(\mathcal{T}_{N}\right) d V_{g}$ since the function $u_{N}$ is $C^{4}$. The calculations of section 6.2 , performed with $u_{N}$ instead of $u$, imply that $d F\left[u_{N}\right]\left(\mathcal{T}_{N}\right)=Q_{N}-\Delta \tilde{\psi}_{N}$. We have singled out the function $\tilde{\psi}$ throughout this argument because there is no $C^{0}$ convergence available for the sequence $\left(\Delta \tilde{\psi}_{N}\right)_{N \in \mathbb{N}}$ whereas there is one for the sequence $\left(Q_{N}\right)_{N \in \mathbb{N}}$. We are thus led to write:

$$
I_{v}\left[u_{N}\right]=\int_{M}\left(v Q_{N}-\nabla_{k} v \nabla^{k} \tilde{\psi}_{N}\right) d V_{g}
$$

Letting the integer $N$ go to $\infty$ and recalling the various $C^{0}$ and $C^{1}$ limits recorded above, we infer that:

$$
I_{v}[u]=\int_{M}\left(v Q[u]-\nabla_{k} v \nabla^{k} \tilde{\psi}\right) d V_{g}
$$

or else, since the function $\tilde{\psi}$ is $C^{2}$ :

$$
I_{v}[u]=\int_{M} v(Q[u]-\Delta \tilde{\psi}) d V_{g}
$$

Now, this equality holds for every function $v \in C^{1}$, so the claim is proved

Proposition A.1 tells us that, with a $C^{3}$ Riemannian metric having a $C^{2}$ curvature, and with $C^{2}$ positive probability measures, all the results of the paper hold and yield $C^{3, \alpha}$ potential functions $u\left(\mu_{0}, \mu_{1}\right)$, for every $\alpha \in(0,1)$. In particular, regarding our preceding discussion, the following extension of Theorem 1.4 is now known to hold:

Theorem A.1. Let $g$ be a $C^{3}$ Riemannian metric on $\mathbb{S}^{2}$ with Gauss curvature $K$ of class $C^{2}$ satisfying the normalization $\min _{\mathbb{S}^{2}} K=1$. There exists a universal real $\epsilon>0$ such that, if $|K-1|_{C^{2}} \leqslant \epsilon$, for every $\alpha \in(0,1)$ and every couple $\left(\mu_{0}, \mu_{1}\right)$ of $C^{2}$ positive probability measures on $\mathbb{S}^{2}$, the potential function of the optimal transportation map $\mathrm{Mc}\left(\mu_{0}, \mu_{1}\right)$ is of class $C^{3, \alpha}$.

\section{References}

[Aub98] Thierry Aubin. Some nonlinear problems in Riemannian geometry. Springer Monographs in Mathematics. Springer-Verlag, Berlin, 1998. 
[Bes78] Arthur L. Besse. Manifolds all of whose geodesics are closed, volume 93 of Ergebnisse der Mathematik und ihrer Grenzgebiete [Results in Mathematics and Related Areas]. Springer-Verlag, Berlin, 1978. With appendices by D. B. A. Epstein, J.-P. Bourguignon, L. Bérard-Bergery, M. Berger and J. L. Kazdan.

[Bre87] Yann Brenier. Décomposition polaire et réarrangement monotone des champs de vecteurs. C. R. Acad. Sci. Paris Sér. I Math., 305(19):805-808, 1987.

[Bre91] Yann Brenier. Polar factorization and monotone rearrangement of vector-valued functions. Comm. Pure Appl. Math., 44(4):375417, 1991.

[Cab97] Xavier Cabré. Nondivergent elliptic equations on manifolds with nonnegative curvature. Comm. Pure Appl. Math., 50(7):623-665, 1997.

[Car51] Élie Cartan. Leçons sur la géométrie des espaces de Riemann. Gauthier-Villars, Paris, 1951. 2d ed.

[Car67] Henri Cartan. Calcul différentiel. Hermann, Paris, 1967.

[CE08] Jeff Cheeger and David G. Ebin. Comparison theorems in Riemannian geometry. AMS Chelsea Publishing, Providence, RI, 2008. Revised reprint of the 1975 original.

[Cha70] Isaac Chavel. On Riemannian symmetric spaces of rank one. Advances in Math., 4:236-263 (1970), 1970.

[CMS01] Dario Cordero-Erausquin, Robert J. McCann, and Michael Schmuckenschläger. A Riemannian interpolation inequality à la Borell, Brascamp and Lieb. Invent. Math., 146(2):219-257, 2001.

[Cor99] Dario Cordero-Erausquin. Sur le transport de mesures périodiques. C. R. Acad. Sci. Paris Sér. I Math., 329(3):199202, 1999.

[Cri62] Richard J. Crittenden. Minimum and conjugate points in symmetric spaces. Canad. J. Math., 14:320-328, 1962.

[dC92] Manfredo Perdigão do Carmo. Riemannian geometry. Mathematics: Theory \& Applications. Birkhäuser Boston Inc., Boston, MA, 1992. Translated from the second Portuguese edition by Francis Flaherty.

[Del81] Philippe Delanoë. Équations du type de Monge-Ampère sur les variétés riemanniennes compactes. II. J. Funct. Anal., 41(3):341353, 1981. 
[Del82] Philippe Delanoë. Équations du type de Monge-Ampère sur les variétés riemanniennes compactes. III. J. Funct. Anal., 45(3):403-430, 1982.

[Del03] Philippe Delanoë. Local solvability of elliptic, and curvature, equations on compact manifolds. J. Reine Angew. Math., 558:2345, 2003.

[Del04] Philippe Delanoë. Gradient rearrangement for diffeomorphisms of a compact manifold. Differential Geom. Appl., 20(2):145-165, 2004

[DG10] Philippe Delanoë and Yuxin Ge. Regularity of optimal transport on compact, locally nearly spherical, manifolds. J. Reine Angew. Math., 646:65-115, 2010.

[DG11] Philippe Delanoë and Yuxin Ge. Locally nearly spherical surfaces are almost-positively c-curved. Methods Appl. Anal., 18(3):269$302,2011$.

[DL06] Philippe Delanoë and Grégoire Loeper. Gradient estimates for potentials of invertible gradient-mappings on the sphere. Calc. Var. Partial Differential Equations, 26(3):297-311, 2006.

[DR13] Philippe Delanoë and François Rouvière. Positively curved riemannian locally symmetric spaces are positively squared distance curved. Canad. J. Math., 65(4):757-767, 2013.

[Eva82] Lawrence C. Evans. Classical solutions of fully nonlinear, convex, second-order elliptic equations. Comm. Pure Appl. Math., 35(3):333-363, 1982.

[Fig12] Alessio Figalli, June 2012. Private communication.

[FKM13a] Alessio Figalli, Young-Heon Kim, and Robert J. McCann. Hölder continuity and injectivity of optimal transport maps. Arch. Ration. Mech. Anal., 209(3):747-795, 2013.

[FKM13b] Alessio Figalli, Young-Heon Kim, and Robert J. McCann. Regularity of optimal transport maps on multiple products of spheres. J. Eur. Math. Soc., 15(4):1131-1166, 2013.

[FR09] Alessio Figalli and Ludovic Rifford. Continuity of optimal transport maps and convexity of injectivity domains on small deformations of $\mathbb{S}^{2}$. Comm. Pure Appl. Math., 62(12):1670-1706, 2009.

[FRV10] Alessio Figalli, Ludovic Rifford, and Cédric Villani. On the MaTrudinger-Wang curvature on surfaces. Calc. Var. Partial Differential Equations, 39(3-4):307-332, 2010. 
[FRV11] Alessio Figalli, Ludovic Rifford, and Cédric Villani. Necessary and sufficient conditions for continuity of optimal transport maps on Riemannian manifolds. Tohoku Math. J. (2), 63(4):855-876, 2011.

[FRV12] Alessio Figalli, Ludovic Rifford, and Cédric Villani. Nearly round spheres look convex. Amer. J. Math., 134(1):109-139, 2012.

[Gir30] Georges Giraud. Sur différentes questions relatives aux équations du type elliptique. Ann. Sci. École Norm. Sup. (3), 47:197-266, 1930.

[Gra04] Alfred Gray. Tubes, volume 221 of Progress in Mathematics. Birkhäuser Verlag, Basel, second edition, 2004. With a preface by Vicente Miquel.

[GT01] David Gilbarg and Neil S. Trudinger. Elliptic partial differential equations of second order. Classics in Mathematics. SpringerVerlag, Berlin, 2001. Reprint of the 1998 edition.

[Har02] Philip Hartman. Ordinary differential equations, volume 38 of Classics in Applied Mathematics. Society for Industrial and Applied Mathematics (SIAM), Philadelphia, PA, 2002. Corrected reprint of the second (1982) edition [Birkhäuser, Boston, MA; MR0658490 (83e:34002)], With a foreword by Peter Bates.

[Hop27] Eberhard Hopf. Elementare Bemerkungen über die Lösungen partieller Differentialgleichungen zweiter Ordnung vom elliptischen Typus. Sitz. Ber. Preuss. Akad. Wissensch. Berlin, Math.Phys. Kl., 19:147-152, 1927.

[Hop31] Eberhard Hopf. Über den funktionalen, insbesondere den analytischen Charakter der Lösungen elliptischer Differentialgleichungen zweiter Ordnung. Math. Z., 34:194-233, 1931.

[Jos11] Jürgen Jost. Riemannian geometry and geometric analysis. Universitext. Springer, Heidelberg, sixth edition, 2011.

[Kar88] Hermann Karcher. A geometric classification of positively curved symmetric spaces and the isoparametric construction of the Cayley plane. Astérisque, (163-164):111-135, 1988. On the geometry of differentiable manifolds (Rome, 1986).

[Kim08] Young-Heon Kim. Counterexamples to continuity of optimal transport maps on positively curved Riemannian manifolds. Int. Math. Res. Not. IMRN, pages Art. ID rnn120, 15, 2008. 
[Kli95] Wilhelm P. A. Klingenberg. Riemannian geometry, volume 1 of de Gruyter Studies in Mathematics. Walter de Gruyter \& Co., Berlin, second edition, 1995.

[KM10] Young-Heon Kim and Robert J. McCann. Continuity, curvature, and the general covariance of optimal transportation. J. Eur. Math. Soc. (JEMS), 12(4):1009-1040, 2010.

[KM12] Young-Heon Kim and Robert J. McCann. Towards the smoothness of optimal maps on Riemannian submersions and Riemannian products (of round spheres in particular). J. Reine Angew. Math., 664:1-27, 2012.

[KN96a] Shoshichi Kobayashi and Katsumi Nomizu. Foundations of differential geometry. Vol. I. Wiley Classics Library. John Wiley \& Sons Inc., New York, 1996. Reprint of the 1963 original, A Wiley-Interscience Publication.

[KN96b] Shoshichi Kobayashi and Katsumi Nomizu. Foundations of differential geometry. Vol. II. Wiley Classics Library. John Wiley \& Sons Inc., New York, 1996. Reprint of the 1969 original, A Wiley-Interscience Publication.

[Lan02] Serge Lang. Introduction to differentiable manifolds. Universitext. Springer-Verlag, New York, second edition, 2002.

[Loe09] Grégoire Loeper. On the regularity of solutions of optimal transportation problems. Acta Math., 202(2):241-283, 2009.

[Loe11] Grégoire Loeper. Regularity of optimal maps on the sphere: the quadratic cost and the reflector antenna. Arch. Ration. Mech. Anal., 199(1):269-289, 2011.

[LTW10] Jiakun Liu, Neil S. Trudinger, and $\mathrm{Xu}-$ Jia Wang. Interior $C^{2, \alpha}$ regularity for potential functions in optimal transportation. Comm. Partial Differential Equations, 35(1):165-184, 2010.

[LV10] Grégoire Loeper and Cédric Villani. Regularity of optimal transport in curved geometry: the nonfocal case. Duke Math. J., 151(3):431-485, 2010.

[McC01] Robert J. McCann. Polar factorization of maps on Riemannian manifolds. Geom. Funct. Anal., 11(3):589-608, 2001.

[Mil97] John W. Milnor. Topology from the differentiable viewpoint. Princeton Landmarks in Mathematics. Princeton University Press, Princeton, NJ, 1997. Based on notes by David W. Weaver, Revised reprint of the 1965 original. 
[Mon81] Gaspard Monge. Mémoire sur la théorie des déblais et remblais. Mémoires Acad. Royale Sci. Paris, 1781.

[MTW05] Xi-Nan Ma, Neil S. Trudinger, and Xu-Jia Wang. Regularity of potential functions of the optimal transportation problem. Arch. Ration. Mech. Anal., 177(2):151-183, 2005.

[Mye35] Sumner Byron Myers. Connections between differential geometry and topology. I. Simply connected surfaces. Duke Math. J., 1(3):376-391, 1935.

[PT88] Richard S. Palais and Chuu-Lian Terng. Critical point theory and submanifold geometry, volume 1353 of Lecture Notes in Mathematics. Springer-Verlag, Berlin, 1988.

[PW67] Murray H. Protter and Hans F. Weinberger. Maximum principles in differential equations. Prentice-Hall Inc., Englewood Cliffs, N.J., 1967.

[Tru83] Neil S. Trudinger. Fully nonlinear, uniformly elliptic equations under natural structure conditions. Trans. Amer. Math. Soc., 278(2):751-769, 1983.

[Tru06] Neil S. Trudinger, June 2006. Private communication.

[TW09] Neil S. Trudinger and $\mathrm{Xu}-$ Jia Wang. On the second boundary value problem for Monge-Ampère type equations and optimal transportation. Ann. Sc. Norm. Super. Pisa Cl. Sci. (5), 8(1):143-174, 2009.

[Vét11] Jérôme Vétois. Continuity and injectivity of optimal maps. Preprint, LJAD, Université Nice Sophia Antipolis, France, 2011.

[Wei68] Alan D. Weinstein. The cut locus and conjugate locus of a riemannian manifold. Ann. of Math. (2), 87(1):29-41, 1968.

[YI73] Kentaro Yano and Shigeru Ishihara. Tangent and cotangent bundles: differential geometry. Marcel Dekker Inc., New York, 1973. Pure and Applied Mathematics, No. 16. 